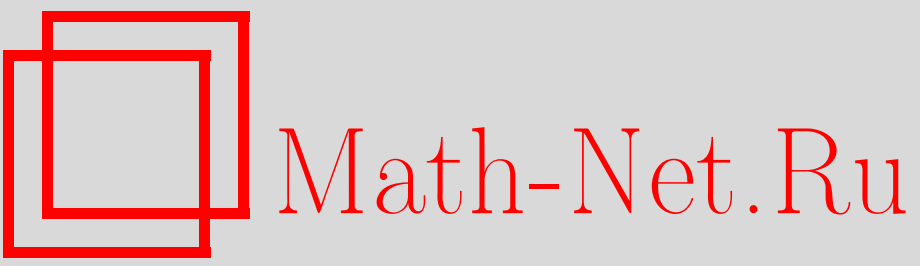

А. Трейбич, Гиперэллиптические касательные накрытия и конечно-зонные потенциалы, УМH, 2001, том 56, выпуск 6, 89-136

DOI: https://doi.org/10.4213/rm454

Использование Общероссийского математического портала Math-Net.Ru подразумевает, что вы прочитали и согласны с пользовательским соглашением

http://www.mathnet.ru/rus/agreement

Параметры загрузки:

IP : 54.80 .97 .219

26 апреля 2023 г., 17:05:58 


\title{
ГИПЕРЭЛЛИПТИЧЕСКИЕ КАСАТЕЛЬНЫЕ НАКРЫТИЯ И КОНЕЧНО-ЗОННЫЕ ПОТЕНЦИАЛЫ
}

\author{
А. ТРЕЙБИч
}

Настоящая работа посвящена изучению конечно-зонных потенциалов, выражаемых через эллиптические функции. Находятся новые эллиптические конечно-зонные потенциалы и новые эллиптические солитоны уравнения КдВ. Сделан новый шаг вперед в изучении взаимосвязей между эллиптическими солитонами $\mathrm{Kд}$, эллиптическими конечно-зонньми потенциалами и якобианами ассоциированных спектральных кривых.

Библиография: 42 названия.

\section{СОДЕРЖАНИЕ}

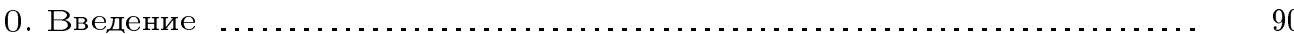

1. Гиперэллиптические касательные накрытия и эллиптические конечно-зонные потенциаль

2. Гиперэллиптические касательные накрытия как дивизоры проективной поверхности $S^{\perp}[38]$

3. О счетности множества исключительных дивизоров на рациональных поверхностях $S^{\sim[38]}$

4. Исключительные накрытия; другие случаи

5. Канонические $\theta$-характеристики и их потенциалы: случай $\mu^{(2)}=2 n+1$ [41]

6. Вывод новых эллиптических конечно-зонных потенциалов

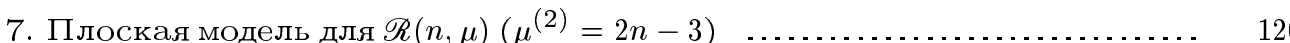

8. Подход эллиптической поверхности в изучении плоской модели $\mathscr{C}_{\vec{a}} \ldots \ldots$

9. Полное описание нового класса четных эллиптических конечно-зонных

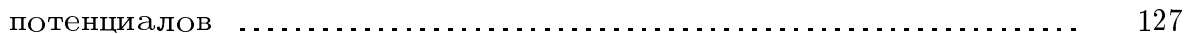

Список литературы . . . . . . . . . . . . . . . . . . .

Предметный указатель . . . . . . . . . . . . . . . 135

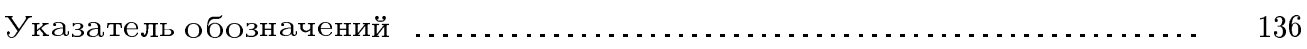




\section{0. Введение}

Интенсивное развитие теории конечно-зонного интегрирования на основе алгебро-геометрических методов, начатое в середине 70-х годов, позволило построить точные решения большого числа дифференциальных уравнений в частных производных. Анализ задачи Коши для уравнения Кортевега-де Вриза $(\mathrm{K}$ дВ) показал, что наиболее интересньми квазипериодическими начальными условиями являются конечно-зонные потенщиалы. Благодаря работе С.П. Новикова [32], где было доказано, что такие потенциалы, а также соответствуюшие решения уравнения $\mathrm{KдВ} \mathrm{определе-}$ ны и эволюционируют на многообразии Якоби римановой поверхности, была осознана их важность для решения всего класса вполне интегрируемых дифференциальных уравнений. На основании этого результата А.Р. Итсом и В.Б. Матвеевым [27] были найдены явные формулы для конечно-зонных решений в терминах абелевых функций; впоследствии формулы такого типа были найдены и для других интегрируемых уравнений. Последующие десятилетия, как и настоящее время, характеризуются неослабевающим интересом к феномену полной интегрируемости, его естественной связи с алгебро-геометрическими конструкциями и его эффективному проникновению в различные области математики и физики. Настояшая работа посвящена изучению конечно-зонных потенциалов, выражаемых через эллиптические функции.

Уместно напомнить, что действительнозначная функция, $q(x)$, является конечнозонным потенциалом тогда и только тогда, когда спектр в $L^{2}(\mathbb{R})$ оператора Хилла, $\partial_{x}^{2}-q(x)$, содержит конечное число зон (и лакун). Общий комплекснозначньй мероморфньй конечно-зонньй потенциал, порождающий стационарные решения иерархии КдВ и построенный по гиперэллиптической римановой поверхности, определяется следуюшим образом.

Пусть $L=\partial_{x}^{2}-u(x, t)$ - дифференциальньй оператор второго порядка, зависящий от параметра $t$, и $g$ - натуральное число. Тогда существует дифференциальньй оператор $P$ порядка $2 g+1$, коэффициентами которого являются дифференщиальные полиномы от $u$, такой, что коммутатор $[P, L]$ - оператор умножения (см., например, [33]). Отсюда следует, что (высшее) уравнение $\mathrm{K} д \mathrm{~B}, u_{t}=[P, L]$, описьвает изоспектральную деформацию $L(0)$. Кроме того, поскольку $q$ является действительнозначной гладкой функцией, то $q$ - конечно-зонный потенциал тогда и только тогда, когда эта функция удовлетворяет для некоторого $g$ одному из данных вьше уравнений (см. [9], [12], [27], [32]). На этом основании любая такая комплекснозначная мероморфная функция называется конечно-зонным потенциалом. С другой стороны, если дифференциальные операторы $P$ и $L$ коммутируют (т.е. если $[P, L]=0$ ), то известная теорема Бурхнала и Чаунди утверждает, что эти операторы алгебраически зависимы, а именно, сушествует $2 g+1$ таких комплексных чисел $\left\{r_{i}, i=0, \ldots, 2 g\right\}$, что $P^{2}=\Pi_{i}\left(L-r_{i}\right)$ (см., например, [33]). Пусть Г обозначает гиперэллиптическую риманову поверхность, полученную в результате одноточечной компактификашии аффинной комплексной кривой $\left\{(x, y), y^{2}=\Pi_{i}\left(x-r_{i}\right)\right\}$, и пусть $\theta$ обозначает соответствующую тэта-функцию. Любое из описанных вьше решений (высших) уравнений $\mathrm{K} д \mathrm{~B}, u(x, t)$, определяется замечательной формулой Итса-Матвеева (ср. [27])

$$
u(x, t)=-2 \partial_{x}^{2} \ln \theta(x U+t V+W), \quad U, V, W \in \mathbb{C}^{g} .
$$

Обратно, для любых спектральных данных $\left(\Gamma, p, \lambda, D=\sum_{1 \leqslant i \leqslant g} p_{i}\right)$, где Г - гиперэллиптическая Риманова поверхность положительного рода $g, p$ - точка Вейер- 
штрасса на $\Gamma, \lambda$ - локальная координата в окрестности $p$ и $D$ - эффективньй дивизор степени $g$, теория одноточечной функции Бейкера-Ахиезера восстанавливает решение уравнения $\mathrm{K} д \mathrm{~B}, u(x, t)$, в точности в виде $(*)$ (ср. [11]). В частности, для любого комплексного $t_{0}$ функция $u\left(x, t_{0}\right)$ - конечно-зонный потенциал.

Явные примеры двукратно периодических конечно-зонных потенциалов могут быть построены на основе пионерских работ Ч. Эрмита [25] и Е.Л. Айнса [26]. А именно, пусть ю обозначает эллиптическую функцию Вейерштрасса, ассоциированную с прямоугольной решеткой, $\omega^{\prime}$ - мнимьй полупериод и $g$ - натуральное число. В современной спектрально-теоретической интерпретации результаты Айнса приводят к утверждению о том, что самосопряженный оператор $\partial_{x}^{2}-g(g+1) \wp(x+\omega)$ в $L^{2}(\mathbb{R})$ обладает конечно-зонньм спектром; соответствующие спектральные данные были найдены и подробно исследованы (cp. [31]). Последние результаты были затем обобшены на произвольные решетки периодов и общие римановы поверхности (ср. [38]).

Из работ А.Р. Итса и В.Б. Матвеева [27], Б.А. Дубровина [9], Г. Фляшки [16], Г. Маккина и П. ван Мербек [31], Г. Эро, Г. Маккина и Й. Мозера [1] следует, что вещественнозначная (на $\mathbb{R})$ эллиптическая функция $q(x)$ является конечно-зонным потенциалом тогда и только тогда, когда существует эллиптический КдВ-солитон (т.е. двукратно периодическое по $x$ решение уравнения $\mathrm{K} д \mathrm{~B}), u(x, t)$, причем $q(x)=$ $u(x, 0)$. Этот результат остается в силе и для комплексно-аналитической функции $q(x)$ (cp. [38]), которая характеризует эллиптический конечно-зонньй потенциал, как начальное условие для эллиптического солитона $u(x, t)$ уравнения $\mathrm{K}$ дВ.

Вернемся к примеру $g$-зонной функции Хилла или Ламе $q(x)=g(g+1) \wp(x)$. Ее эволюция в силу уравнения $\mathrm{K}$ дВ (cp. [12] для $g=2$ и [1] для произвольного $g$ ) определяется по формуле

$$
u(x, t) \equiv 2 \sum_{1 \leqslant i \leqslant n} \wp\left(x-x_{i}(t)\right), \quad n:=\frac{g(g+1)}{2},
$$

где полюса $x_{i}(t)$ сливаются при $t=0$ и описьвают интегрируемую систему Калоджеро-Мозера с взаимодействием вида $\wp\left(x_{i}-x_{j}\right)$. Отсюда, в частности, следует, что формула Итса-Матвеева $(*)$, выражающая $u(x, t)$ через $g$-мерные тэта-функции, сводится к сумме эллиптических функций.

$\mathrm{C}$ другой стороны, хорошо известные многосолитонные решения уравнения $\mathrm{K}$ дВ представляют собой нелинейную суперпозицию солитонов. Подобная редукция формулы $(*)$ к сумме эллиптических функций (или комбинации абелевых функций младших родов) представляется интересной, поскольку позволяет:

1) описывать специальные решения уравнения $\mathrm{K}$ дВ (в частности, эллиптические по одной из переменных);

2) изучать и решать естественным образом связанные интегрируемые системы частиц типа эллиптической системы Калоджеро-Мозера.

С геометрической точки зрения объектом исследования являются многообразия Якоби, которые распадаются на сумму абелевых подмногообразий младших родов. Проблема описания многообразий Якоби, связанных с динамикой системы КдВ, была сформулирована С. П. Новиковым в [32]. Для ее решения были привлечены такие методы, как теория редукции Пуанкаре-Вейерштрасса абелевых функций к младшим родам (см. [5]), а также редукция абелевых функций кривых, обладаюших нетривиальньми автоморфизмами (см. [3], [4]), в результате чего были построены примеры 
редукции к эллиптическим функциям решений ряда интегрируемых уравнений в частных производных.

Восходящее к Г. Альфану [24] изучение спектральных свойств дифференциальных операторов произвольного порядка над полем эллиптических функций является другим естественным подходом в этом контексте. Этот подход позволяет исследовать другие редукции уравнения Кадомцева-Петвиашвили, ассоциированные с негиперэллиптическими римановьми поверхностями, в частности, уравнение Буссинеска; в [8], [13], [14], [42] этим методом был получен ряд весьма нетривиальных примеров.

Однако эта статья посвящена, в основном, нахождению новых эллиптических конечно-зонных потенциалов и новых эллиптических солитонов уравнения КдВ. Каждое такое решение ассоциируется с некоторьми спектральными данньми $(\Gamma, p, \lambda, D)$, описанными выше (ср. [38; теорема 2.7]), при которых первьй КдВ-поток генерируется касательньм вектором к Г в точке $p$ внутри многообразия Якоби. Поэтому для того чтобы получить эллиптическое решение $\mathrm{K} д \mathrm{~B}$, мы должны начать с решения следуюшей геометрической задачи:

найти все такие отмеченные римановы поверхности, $(\Gamma, p)$, что внутри их многообразий Якоби Г является касательной в точке $р$ к әллиптической кривой.

Упомянутая вьше теория редукции также может быть применена для решения этой специальной проблемы, как это было показано в [6], [7], где были получены частичные ответы.

В 1980 году И. Кричевер предложил и развил иной весьма эффективньй подход, основанный на его прежних результатах, относящихся к теории одноточечной функции Бейкера-Ахиезера (ср. [28]-[30]). Отправным пунктом в этой теории является заданная эллиптическая кривая $X$, для которой строятся все римановы поверхности, разрешаюшие сфформулированную вьше геометрическую задачу и представляюшие собой специальные накрытия эллиптической кривой $X$, удовлетворяющие критерию касательности. Каждый такой ковектор, $\pi: \Gamma \rightarrow X$, порождает эллиптический КР-солитон (т.е. решение уравнения Кадомцева-Петвиашвили, являющееся двукратно-периодическим по $x$ ). Более того, существует полином степени $n=\operatorname{deg} \pi$, коэффициентами которого являются эллиптические на $X$ функции,

$$
P(T, z)=\sum_{0 \leqslant i \leqslant n} \alpha_{i}(z) T^{i}
$$

такой, что

$$
\left\{(T, z) \in \mathbb{P}^{1}(\mathbb{C}) \times X, P(T, z)=0\right\},
$$

т.е. многообразие его нулей бирационально эквивалентно Г (ср. [30]); указанные полиномы допускают как простое описание, так и алгоритмическое построение (ср. [36]). Тщательное изучение этого многообразия нулей позволяет понять, как выделить (по крайней мере, в принципе) накрытия, приводящие к эллиптическим солитонам КдВ. Явные примеры таких гиперэллиптических римановых поверхностей вплоть до рода 8 были построены в конще 80-х годов (ср. [34]-[36]).

Отметим, что описанные римановы поверхности естественно вкладьваются в специальную линейчатую поверхность над $X$, что позволяет усовершенствовать подход 
Кричевера на основе использования результатов теории алгебраических поверхностей. Это позволило найти дискретное семейство накрытий над $X$, приводящих к эллиптическим солитонам $\mathrm{K} д \mathrm{~B}$ и, в частности, - к потенциалам Хилла-Ламе. Для вычисления соответствуюших (четных) эллиптических конечно-зонных потенциалов требуется формула для кратности пересечения тэта-дивизора на многообразии Якоби с произвольным первым KдВ-потоком. Такая формула, действительно, существует, что позволяет сделать заключительный шаг в доказательстве того, что для любого целочисленного вектора $\left(a_{0}, \ldots, a_{3}\right)$ функция

$$
\sum_{0 \leqslant i \leqslant 3} a_{i}\left(a_{i}+1\right) \wp\left(x-\omega_{i}\right)
$$

где $\left\{\omega_{i}, i=0, \ldots, 3\right\}$ - множество полупериодов $X$, является эллиптическим конечно-зонньм потенциалом (ср. [18]-[20], [38], [41]).

В настоящей статье мы продвигаемся на один шаг вперед в изучении взаимосвязей между эллиптическими солитонами $\mathrm{K} д \mathrm{~B}$, эллиптическими конечно-зонными потенциалами и якобианами ассоциированных спектральных кривых, а именно, мы находим другое семейство накрытий над $X$, приводяших к эллиптическим солитонам $\mathrm{K} д \mathrm{~B}$, и описываем явно канонически ассоциированные эллиптические конечно-зонные потенциалы.

Для краткого описания работы мы сначала приведем основные определения, объясняя их взаимосвязь, и сформулируем основные результаты. Строгие определения будут даны в последуюших разделах.

Зафиксируем решетку $\Lambda \subset \mathbb{C}$ и обозначим через $\wp$ стандартную $\Lambda$-периодическую функцию Вейерштрасса и через ъ' - ее производную; обозначим через $(X, q)$ эллиптическую кривую $(\mathbb{C} / \Lambda, 0)$. Обозначим через $\operatorname{KdV}(n, X)(n>0)$ замкнутое подмногообразие $n$-симметрических степеней $X, X_{n}$, определяемых уравнениями:

$$
\sum_{1 \leqslant j \leqslant n, j \neq i} \wp^{\prime}\left(x_{i}-x_{j}\right)=0, \quad i=1, \ldots, n .
$$

Хорошо известно, что мероморфная функция $u(x, t), \Lambda$-периодическая по $x$, является эллиптическим солитоном $\mathrm{K} д \mathrm{~B}$ тогда и только тогда, когда существуют такие натуральное число $n \in \mathbb{N}$ и отображение $\mathbb{C} \rightarrow \operatorname{KdV}(n, X), t \mapsto\left\{x_{i}(t)\right\}$, что с точностью до функции от $t$

$$
u(x, t) \equiv 2 \sum_{1 \leqslant i \leqslant n} \wp\left(x-x_{i}(t)\right)
$$

(cp. [1]). При этом каждый эллиптический КдВ-солитон единственным образом ассошиируется с проективной кривой рода $g>0,(\Gamma, p)$, снабженной дивизором степени $g, D_{0}$, и проекщией $\pi: \Gamma \rightarrow X$ (называемой далее гиперәллиптическим касательнымм накрытием), которая обладает следуюшими свойствами:

I) Г - гиперэллиптическая кривая и $p$ - ее гладкая точка Вейерштрасса (т.е. существует мероморфная функция $f: \Gamma \rightarrow \mathbb{P}^{1}(\mathbb{C})$, имеющая в точке $p$ единственньй полюс второго порядка);

II) канонические образы $(\Gamma, p)$ и $(X, q)$ в якобиане $\Gamma$ касаются в начале координат. 
Обратно, для любого дивизора $D$ степени $g$ решение уравнения $\mathrm{K}$ дВ, соответствующее спектральным данным $(\Gamma, p, D)($ cp. $[10 ; \S 3.1],[33 ; \S 6])$, является эллиптическим $\mathrm{K}$ дВ-солитоном (совпадающим с эллиптическим солитоном, определяемым дивизоpом $\left.D=D_{0}\right)$.

Известно еще одно важное свойство гиперэллиптических касательных накрытий. Обозначим через $u_{D}(x, t)$ эллиптический $\mathrm{K}$ дВ-солитон, построенный по спектральным данным $(\pi, D)$, как это было описано вьше. Сушествует функция (так назьваемая функция Бейкера-Ахиезера), мероморфная на $\mathbb{C} \times(\Gamma \backslash\{p\})$, обозначаемая как $\psi_{D}(x, \lambda)$ и обладающая следуюшими свойствами:

i) $\forall \lambda \in \Gamma \backslash\{p\}$ удовлетворяется уравнение

$$
\partial_{x}^{2}\left(\psi_{D}(x, \lambda)\right)-u_{D}(x, 0) \psi_{D}(x, \lambda)=f(\lambda) \psi_{D}(x, \lambda)
$$

ii) $\forall \omega \in \Lambda, \psi(x+\omega, \lambda)-\operatorname{кратно~} \psi(x, \lambda)$.

Таким образом, аффинная кривая $\Gamma \backslash\{p\}$ может быть описана как совместньй спектр оператора $\partial_{x}^{2}-u_{D}(x, 0)$ и оператора сдвига, $\mathbf{T}_{\omega}: \xi(x) \rightarrow \xi(x+\omega), \omega \in \Lambda$. Отсюда также следует, что функция $u_{D}(x, 0)$ (а также $\left.2 \sum_{1 \leqslant i \leqslant n} \wp\left(x-\alpha_{i}\right), \forall\left\{\alpha_{i}\right\} \in \operatorname{KdV}(n, X)\right)$ является эллиптическим конечно-зонньм потенциалом.

В том случае, когда дивизор $D$ линейно эквивалентен $g \cdot p$, четная функция $u_{D}(x, 0)$ будет назьваться порождающим потенииалом степени $n$ над $X(n \in \mathbb{N})$. Эти потенциалы описьвают все эллиптические конечно-зонные потенщиалы с точностью до их изоспектральной эквивалентности (т.е. различные порождаюшие потенщиалы соответствуют неизоморфньм спектральным данным).

Представляется, однако, затруднительным построить конкретные примеры эллиптических конечно-зонных потенциалов непосредственно по данному выше описанию, не восстановив спектральных данных, или определить, являются ли два таких потенциала изоспектральными. С другой стороны, однако, известно, что для любого $n \in \mathbb{N}^{*}$ сушествует конечное число образуюших потенциалов степени $n$ над $X$ [39]. Поэтому их классификация является более легкой проблемой.

Мы описываем далее успешно выполненную двухэтапную программу получения больших семейств порождаюших потенциалов. Несмотря на то, что исчерпьваюшее решение проблемы еше не получено, мы приведем наши предположения относительно такой полной классификации.

\section{"2-этапная программа":}

А) построить все гиперэллиптические касательные накрытия;

В) построить соответствуюшие порождаюшие потенциалы и выделить их из множества четных эллиптических функций.

Вьполнение этой двухэтапной программы было начато в [38], где были изучены основные свойства гиперэллиптических касательных накрытий. Как оказалось, для любой решетки $\Lambda$ и любой степени $n$ существует только конечное число таких накрытий, причем они факторизуются раздутиями некоторых линейчатых поверхностей над $X=\mathbb{C} / \Lambda$, обозначаемых как $S^{\perp} \rightarrow X$. При этом $S^{\perp}$ проектируется на рациональную поверхность $S \sim$, обладающую тем свойством, что все гиперэллиптические касательные накрытия над $X$ возникают из рациональных неприводимых дивизоров на $S^{\sim}$. Поэтому построенные таким образом гиперэллиптические касательные накрытия, названные в [39] исключительными, соответствуют прообразам проекций 
$S^{\perp} \rightarrow S^{\sim}$ исключительных дивизоров $S^{\sim}$, что объясняет происхождение указанного названия.

Следующий этап программы состоит в выводе порождающего потенщиала, соответствуюшего исключительному накрытию $\pi:(\Gamma, p) \rightarrow(X, q)$. Благодаря формуле Итса-Матвеева $(*)$ вьполнение второго этапа сводится к нахождению пересечения тэта-дивизора на якобиане Г с образом $(X, q)$. Примечательным фактом является то, что общая формула кратности пересечения Фэя-Сигала-Вильсона [16], [34] оказывается эффективно применимой и в том случае, когда $\pi$ - исключительное накрытие, что является основой при доказательстве следующего результата [41]:

Теорема 0.1. Пусть $\Lambda$ - произвольная решетка, $\left\{\omega_{i}, i=0, \ldots, 3\right\}$-множество полупериодов әллиптической кривой $\mathbb{C} / \Lambda\left(\omega_{0}=0\right)$. Тогда для любьх четырех чисел $\left(a_{i}\right) \in \mathbb{N}^{4} \backslash\{\overrightarrow{0}\}$ функиия

$$
\sum_{0 \leqslant i \leqslant 3} a_{i}\left(a_{i}+1\right) \wp\left(x-\omega_{i}\right)
$$

является четным $\Lambda$-периодическим конечно-зонным потенциалом, ассоциированным с исключительным накрытием степени

$$
n:=\frac{1}{2} \sum_{0 \leqslant i \leqslant 3} a_{i}\left(a_{i}+1\right)
$$

над $\mathbb{C} / \Lambda$ и рода

$$
g:=\frac{1}{2} \max \left\{2 M, 1+\sum-\left(1+(-1)^{\Sigma}\right)\left(m+\frac{1}{2}\right)\right\},
$$

$2 \partial e$

$$
M:=\max \left\{a_{i}\right\}, \quad \sum:=\sum_{0 \leqslant i \leqslant 3} a_{i} \quad \text { u } m:=\min \left\{a_{i}\right\} .
$$

При этом $\sum_{0 \leqslant i \leqslant 3} a_{i}\left(a_{i}+1\right) \wp\left(x-\omega_{i}\right)$ является порождающим потенииалом тогда и только тогда, когда $a_{0}=M$ и $2 M \geqslant \sum-\left(1+(-1)^{\Sigma}\right) m$.

Отметим, что критерий выделения эллиптических конечно-зонных потенциалов из общих в виде условий обрашения в нуль некоторых тэта-констант был получен Е.Д. Белоколосом и В.З. Энольским в [6]; иной, но близкий подход был развит Л. Гавриловьм и А. М. Переломовьм [17]. Недавно Ф. Гестези и Р. Вайкард нашли эффективное аналитическое описание всех эллиптических конечно-зонных потенциалов, которое они применили для доказательства приведенного вьше результата. А именно, ими доказано, что эллиптическая функция $q(x)$ является конечно-зонной тогда и только тогда, когда для любого $E \in \mathbb{C}$ ассоциированное уравнение Хилла, $\psi^{\prime \prime}(x)-q(x) \psi(x)=E \psi(x)$, обладает мероморфной системой решений (см. [19]-[21]). Таким образом, задача определения того, является ли данная эллиптическая функция конечно-зонньм потенциалом, была решена хорошо известным методом Фробениуca. В частности, упомянутьй результат выводится немедленно. Помимо этого они предъявили конкретную процедуру построения соответствуюшей отмеченной римановой поверхности $(\Gamma, p)$, реализованной как накрытие степени 2 над сфферой 
Римана, для некоторых классов эллиптических конечно-зонных потенциалов (см. [18], [21], [22]). Следует также упомянуть, что А.О. Смирнов построил примеры эллиптических конечно-зонных потенциалов, которые не покрываются теоремой 0.1 (см., например, [34]).

Будучи отчасти мотивированы последним упомянутьм результатом, мы развиваем в этой статье описанньй вьше дву хэтапный алгебро-геометрический подход, $(A+B)$, резюмируем известные результаты [41] и рассматриваем первьй пример эллиптического конечно-зонного потенциала, не связанного с исключительными накрытиями. Римановыповерхности, возникаюшие в этапе А), соответствуют в этом случае неприводимьм особым дивизорам рациональной поверхности $S^{\sim}$, имеющей арифметический род 1 (не 0, как ранее). Они могут быть выделены как рациональные слои соответствующего пучка эллиптических кривых в $S^{\sim}$ с помошью формулы, принадлежащей С. Лефшецу. Упоминавшаяся ранее формула Фэя-Сигала-Вильсона, по-прежнему, применима и приводит к новому семейству эллиптических конечно-зонных потенциалов и порождаюших потенциалов.

Наконец, варьируя решетку $\Lambda$ и применяя такую же технику, как и на этапе А), мы получаем полное описание последнего семейства. А именно, мы доказываем следующую теорему.

ТЕОрема 0.2. Для любой решетки $\Lambda$ и любых четырех чисел $\left(a_{i}\right) \in \mathbb{N}^{4} \backslash\{\overrightarrow{0}\}$ четная $\Lambda$-периодическая функиия $2 \wp(x-\rho)+2 \wp(x+\rho)+\sum_{0 \leqslant i \leqslant 3} a_{i}\left(a_{i}+1\right) \wp\left(x-\omega_{i}\right)$ $(2 \rho \neq 0)$ является конечно-зонным потенииалом тогда и только тогда, когда $\rho$ удовлетворяет следующему уравнению: $\sum_{0 \leqslant i \leqslant 3}\left(2 a_{i}+1\right)^{2} \wp^{\prime}\left(\rho-\omega_{i}\right)=0$. При этом для каждого конечно-зонного потенциала род соответствующей римановой поверхности может быть определен по числам $\left(a_{i}\right)$ и $\rho$.

Наконец, мы описываем, как и в [41], подсемейство порождающих потенциалов.

Мы напоминаем для удобства читателя в разделах 1-3 и 5 основные определения и излагаем ранние результаты совместных работ с Жаном-Луи Вердье.

Автор хотел бы подчеркнуть, что за исключением разделов 8 и 9 все остальные разделы обсуждались и разрабатывались совместно с Ж.-Л. Верде (более 9 лет тому назад!). Следует также отметить, что исходные положения для полного описания эллиптических солитонов, полученные в разделе 9 (в частности, основное уравнение (7) и подход плоской модели, обсуждаемьй в разделе 7), также принадлежат ему. Наконец, подчеркнем, что разделы 4 и 7 частично основаны на рукописных заметках Ж.-Л. Вердье. При других обстоятельствах эта совместная работа могла бы быть завершена много лет тому назад.

\section{1. Гиперэллиптические касательные накрытия и эллиптические конечно-зонные потенциалы}

1.1. Пусть $\Gamma$ - неприводимая проективная кривая арифметического рода $g>0$ и $\lambda$ - локальная координата Г в гладкой точке $p \in \Gamma$. Зададим произвольньй неспециальньй дивизор $D$ степени $g$ и найдем такое наибольшее натуральное число $m$, что $D-m p$ все еше остается эффективньм дивизором. Сушествует единственная функция (так назьваемая функиия Бейкера-Ахиезера), обозначаемая $\psi_{D}$, которая мероморфна на $\mathbb{C}^{3} \times(\Gamma \backslash\{p\})$ и удовлетворяет следующим условиям (см. [28], [29] и также $[10],[11])$ : для любой точки общего положения $(x, y, t) \in \mathbb{C}^{3}$ ограничение $\psi_{D}$ на 
$\{(x, y, t)\} \times(\Gamma \backslash\{p\})$ имеет:

a) дивизор полюсов, равньй $D-m p$;

b) существенную особенность в $p$, в окрестности которой справедливо разложение

$$
\psi_{D}(x, y, t ; \lambda)=\frac{1}{\lambda^{m}} \exp \left(x \lambda^{-1}+y \lambda^{-2}+t \lambda^{-3}\right)\left(1+\sum_{j \geqslant 1}^{\infty} \xi_{j, D}(x, y, t) \lambda^{j}\right) .
$$

1.2. Назовем далее $(\Gamma, p)$ гиперәллиптической отмеченной кривой, если существует такая проекщия $f: \Gamma \rightarrow \mathbb{P}^{1}(\mathbb{C})$, что $f^{*}(\infty)=2 p$. В этом случае $\Gamma$ канонически снабжена гиперэллиптической инволюцией, $\sigma: \Gamma \rightarrow \Gamma\left(\Gamma / \sim \sigma \simeq \mathbb{P}^{1}(\mathbb{C})\right)$, для которой точка $p$ является неподвижной. Таким образом, $p$ - точка Вейерштрасса и для любой $\sigma$-антиинвариантной локальной координаты $\Gamma$ в $p$, например $\lambda$, вьполняется

$$
\sigma^{*}(\lambda)=-\lambda \text { и } f(\lambda)=\frac{1}{\lambda^{2}}+O\left(\lambda^{2}\right) .
$$

При этом для любого дивизора $D$, введенного вьше, соответствующая функция Бейкера-Ахиезера удовлетворяет следуюшему дифференциальному уравнению:

$$
\left(\frac{\partial^{2}}{\partial x^{2}}-u_{D}\right) \psi_{D}(x, y, t ; \lambda)=f(\lambda) \psi_{D}(x, y, t ; \lambda),
$$

где

$$
u_{D}(x, y, t):=-\frac{\partial}{\partial x}\left(\xi_{1, D}(x, y, t)\right),
$$

а функция $\psi_{D}$ является так называемой волновой функцией, ассоциированной с обратимым пучком $\mathscr{O}_{\Gamma}(D-p)$ (см. $\left.[33 ; \S 6]\right)$. Во всех случаях $\psi_{D}$ является собственной функцией оператора Шрёдингера, $\frac{\partial^{2}}{\partial x^{2}}-u_{D}$, с собственным значением $f(\lambda)$. При этом функция $u_{D}$ не зависит от $y$ и удовлетворяет уравнению Кортевега-де Вриза. Зафиксировав $\sigma$-антиинвариантную локальную координату $\lambda$ кривой $\Gamma$ в точке $p$, можно единственньм образом определить начальное значение функции $u(x)=u_{D}(x, 0,0)$ по классу изоморфизмов пучка $\mathscr{O}_{\Gamma}(D-p)$. Мы будем назьвать далее $u(x)$ конечно-зонньм потенциалом $\mathrm{KdV}$, ассоциированным с $\left(\Gamma, p, \lambda, \mathscr{O}_{\Gamma}(D-p)\right)$.

1.3. Пусть $\Lambda$ - решетка в $\mathbb{C}$ и $(X, q)=(\mathbb{C} / \Lambda, 0)$ - соответствующая эллиптическая кривая с канонической локальной координатой $z$ в начале координат $q \in X$. Рассмотрим произвольную конечную отмеченную проекцию $\pi:(\Gamma, p) \rightarrow(X, q)$, где $p-$ гладкая точка $\Gamma$ и $\pi(p)=q$. Введем естественно ассоциированное с $\pi$ (рациональное) вложение Абеля, $A_{p}: \Gamma \rightarrow \operatorname{Jac} \Gamma, p^{\prime} \mapsto \mathscr{O}_{\Gamma}\left(p^{\prime}-p\right)$, и гомеоморфизм, $i_{\pi}: X \rightarrow \operatorname{Jac} \Gamma$, $q^{\prime} \mapsto A_{p}\left(\pi^{*}\left(q^{\prime}-q\right)\right)$, Г и $X$ на (обобшенньй) якобиан $\Gamma$.

1.4. ОПРЕДЕЛЕНИЕ [36].

1) Отмеченная проекция $\pi:(\Gamma, p) \rightarrow(X, q)$ является касательныл накрыти$e \mathcal{M}$ тогда и только тогда, когда $i_{\pi}(X)$ и $A_{p}(\Gamma)$ касаются в начале координат, $A_{p}(p)=i_{\pi}(q)$, многообразия Јас $\Gamma$.

2) $\pi:(\Gamma, p) \rightarrow(X, q)$ - гиперэллиптическое касательное накрытие тогда и только тогда, когда $\pi$ - касательное накрытие, а $(\Gamma, p)$ - отмеченная гиперэллиптическая кривая. 
1.5. ТЕОремА (критерий касательности накрытия; [30; с. 288] или [36]). Oтмеченныи миорфизм $\pi:(\Gamma, p) \rightarrow(X, q)$ является касательным накрытием тогда и только тогда, когда существует мероморфная над Г функция $k$, называемая функцией касания, удовлетворяющая следующим условиям:

1) $k$ голоморфна вне $\pi^{-1}(q)$;

2) $k+\pi^{*}\left(z^{-1}\right)$ хорошо определена в окрестности $\pi^{-1}(q) \backslash\{p\}$ и имеет простой полюс в $p$.

1.6. ОПРЕДЕЛЕНИЕ [2]. Пространство модулей всех свободных от кручений когерентных пучков ранга 1 и степени $g-1$ над кривой $\Gamma$, обозначаемое $W(\Gamma)$, сушествует и будет далее называться компактифицированным якобианом кривой Г. Пусть $\omega_{\Gamma}$ обозначает канонический пучок над $\Gamma$, и пусть для любого $\mathscr{L} \in W(\Gamma) \mathscr{L}^{*}$ обозначает пучок $\mathscr{L}^{*}:=\operatorname{Hom}\left(\omega_{\Gamma}, \mathscr{L}\right)$. Тогда $\mathscr{L}^{*}$ принадлежит $W($ Г $)$ и $\left(\mathscr{L}^{*}\right)^{*}$ изоморфен $\mathscr{L}$. Обратно, отображение $\mathscr{L} \mapsto \mathscr{L}^{*}$ порождает инволюцию $W(\Gamma)$. Мы будем говорить, что $\mathscr{L} \in W(\Gamma)-\theta$-характеристика, если $\mathscr{L}$-неподвижная точка определенной вьше инволюции, т.е. если $\mathscr{L} \simeq \operatorname{Hom}\left(\omega_{\Gamma}, \mathscr{L}\right)$.

1.7. ЗАмЕчАния. 1) Компактифицированный якобиан $W(\Gamma)$ является проективным многообразием, на котором обобщенный якобиан, Јас $Г$, действует как тензорное произведение. Если $\Gamma$ - гладкая кривая, то $W(\Gamma)$ совпадает с $\mathrm{Jac}^{g-1}(\Gamma)$. Если, как в нашем случае, Г может быть вложена в гладкую алгебраическую поверхность, то $W(\Gamma)$ неприводим и имеет размерность $g$. Обозначим через $\Gamma^{\circ}$ - открытое гладкое подмножество в $Г$ и через $\Gamma_{g}^{\circ}$ его $g$-ю симметрическую степенғ; тогда многообразие $\mathrm{Jac}^{g-1}(\Gamma)$ совпадает с $\left\{\mathscr{O}_{\Gamma}(D-p), D \in \Gamma_{g}^{\circ}\right\}$ и является плотным открытым подмножеством в $W(\Gamma)$ (см., например, [2]).

2) Мы можем сопоставить некоторьй конечно-зонный потенщиал каждому $\mathscr{L} \in$ $W(\Gamma)$. Действительно, существуют единственная частичная десингуляризация $j: \bar{\Gamma} \rightarrow \Gamma$ арифметического рода $\bar{g} \leqslant g$ и обратимьй пучок $\overline{\mathscr{L}} \in \operatorname{Jac}^{\bar{g}-1}(\bar{\Gamma}) \subset W(\bar{\Gamma})$ такие, что $j_{*}(\overline{\mathscr{L}})$ изоморфно $\mathscr{L}$. Мы будем считать, что соответствуюший потенциал $u_{\mathscr{L}}(x):=u_{\mathscr{L}}(x)$ ассоциирован с $\left.\mathscr{L}[39 ; \S 6]\right)$.

3) Пусть $\pi:(\Gamma, p) \rightarrow(X, q)$ некоторая отмеченная проекция степени $n \geqslant 1$ и

$$
\Theta_{\Gamma}:=\left\{\mathscr{L} \in W(\Gamma), h^{\circ}(\mathscr{L})>0\right\}
$$

- тәта-дивизор $W(\Gamma)$. Для произвольного $\mathscr{L} \in W(\Gamma)$ обозначим

$$
\operatorname{Orb}_{\mathscr{L}}: X \rightarrow W(\Gamma)
$$

соответствуюший орбитныи миорфизм,

$$
q^{\prime} \mapsto \mathscr{L} \otimes i_{\pi}\left(q^{\prime}\right)=\mathscr{L} \otimes \mathscr{O}_{\Gamma}\left(\pi^{*}\left(q^{\prime}-q\right)\right) .
$$

Производя обратное отображение $\Theta_{\Gamma}$ с помошњю Orb $\mathscr{L}$, мы получим дивизор степени $n$ на $X$,

$$
\sum_{i=1}^{n} \alpha_{i}=\operatorname{Orb}_{\mathscr{L}}^{*}\left(\Theta_{\Gamma}\right)
$$

(cp. [39; § А2]). Следовательно, мы можем ассоциировать с каждой ранее определенной проекцией $\pi$ морфизм

$$
I_{\pi}: W(\Gamma) \rightarrow X_{n}, \quad \mathscr{L} \mapsto \operatorname{Orb}_{\mathscr{L}}^{*}\left(\Theta_{\Gamma}\right),
$$

где $X_{n}$ обозначает $n$-ю симметрическую степень $X$. 
1.8. ОПРЕДЕлЕНИЕ. 1) Пусть $\pi:(\Gamma, p) \rightarrow(X, q)$ - гиперэллиптическое касательное накрытие, и пусть $\sigma: \Gamma \rightarrow \Gamma$ обозначает гиперэллиптическую инволющию. Обозначим через $[-1]:(X, q) \rightarrow(X, q)$ обратнцй гомеоморфизм. Тогда $\pi$ эквивариантно (т.е. $\pi \circ \sigma=[-1] \circ \pi$ ) и сушествует единственная $\sigma$-антиинвариантная касательная функция $k$. В частности, можно выбрать $\lambda_{\pi}:=\left(k+\pi^{*}\left(z^{-1}\right)\right)^{-1}$ как $\sigma$-антиинвариантную локальную координату $\Gamma$ в $p$.

2) Напомним, что ю-функция Вейерштрасса, ассоциированная с решеткой $\Lambda$, является единственной четной мероморфной функцией на $X$, голоморфной вне $q$ и имеющей в $q$ лорановское разложение вида $\wp(z)=\frac{1}{z^{2}}+O\left(z^{2}\right)$. Нас будет, в основном, интересовать в $\operatorname{KdV}(n, X)(n \geqslant 1)$ замькание в $X_{n}$ локально замкнутого подмногообразия, определенного следуюшей системой уравнений:

$$
\sum_{\substack{1 \leqslant j \leqslant n \\ j \neq i}} \wp^{\prime}\left(x_{i}-x_{j}\right)=0, \quad i=1, \ldots, n, \quad x_{i} \neq x_{j}\left(\sum_{k=1}^{n} x_{k} \in X_{n}\right)
$$

где $\wp^{\prime}-$ производная $\wp . \mathrm{KdV}(n, X)$ параметризует все $\mathrm{K}$ дВ-решения вида $u(x, t)=$ $2 \sum_{i=1}^{n} \wp\left(x-x_{i}(t)\right)$ и будет далее называться $n$-м $K \partial B$-локусом (над $\left.X\right)$ (см., например, [1]).

1.9. Tеорема $([1],[39 ; \S 7])$. Пусть $\pi:(\Gamma, p) \rightarrow(X, q)$ - зиперэллиптическое касательное накрытие степени $n$. Тогда для любого $\mathscr{L} \in W(\Gamma)$ конечно-зонный потенииал, ассоциированный $c\left(\Gamma, p, \lambda_{\pi}, \mathscr{L}\right)$, равен йL $(x)=2 \sum_{i=1}^{n} \wp\left(x-\alpha_{i}\right)$, где $\sum_{i=1}^{n} \alpha_{i}$ равна $I_{\pi}(\mathscr{L})$ и принадлежит $\mathrm{KdV}(n, X)$. При этом морфизм $I_{\pi}: W(\Gamma) \rightarrow X_{n}$, помимо факторизачии $\mathrm{KdV}(n, X) \rightarrow X_{n}$, является также инбективным и эквивариантным (т.е. для любого $\mathscr{L} \in W(\Gamma) \quad I_{\pi}\left(\mathscr{L}^{*}\right)=$ $\left.[-1]\left(I_{\pi}(\mathscr{L})\right)\right)$. В частности, потенциал иц्L $(x)$ является $\Lambda$-периодическим для любого $\mathscr{L} \in W(\Gamma)$ и четнылм, если и только если $\mathscr{L}-\theta$-характеристика.

1.10. ЗАмечАнИЕ. Пусть $\pi:(\Gamma, p) \rightarrow(X, q)$ такое, как в п. 1.9. Выберем некоторую десингуляризацию $j:(\bar{\Gamma}, p) \rightarrow(\Gamma, p)$ и обозначим через $j_{*}$ замкнутую иммерсию $j_{*}: W(\bar{\Gamma}) \rightarrow W(\Gamma), \overline{\mathscr{L}} \mapsto j_{*}(\overline{\mathscr{L}})$. Тогда композиция отмеченного морфизма, $\bar{\pi}:=\pi \circ j:(\bar{\Gamma}, p) \rightarrow(X, q)$, также является гиперэллиптическим касательным накрытием, и соответствующее инъективное отображение $I_{\bar{\pi}}: W(\bar{\Gamma}) \rightarrow \operatorname{KdV}_{n}(X)$ факторизуется как $I_{\bar{\pi}}=I_{\pi} \circ j_{*}([33 ; \S 6])$. Следовательно, $I_{\bar{\pi}}(W(\bar{\Gamma}))$ - замкнутое подмногообразие $I_{\pi}(W(\Gamma))(\subset \mathrm{KdV}(n, X))$ и семейство $\Lambda$-периодических конечно-зонных потенциалов, ассоциированных с $\pi$, воспроизводит потенциалы, ассоциированные с $\bar{\pi}$.

1.11. ОПРЕДЕЛЕНИЕ. Пусть $\pi:(\Gamma, p) \rightarrow(X, q)$ такое, как в П. 1.9. Назовем $\pi$ мuнимально-гиперэллиптическим касательным накрытием, если $\pi$ не факторизуется (неизоморфньм) гиперэллиптическим касательным накрытием над $X$ такой же степени, как и $\pi$.

1.12. Теорема ([39; $\S 8.10]$, см. также [1]). Пусть $R(n, X)$ обозначает (конечное; см. п. 2.10) множсество классов изоморфизмов минимально-гиперэллиптических касательных накрытий степени $n$ над $X$, и пусть $W(n, X)$ обозначает 
(несвязное) обгединение их компактифицированных якобианов. Тогда естественный морфизм $I_{n}(X): W(n, X) \rightarrow \operatorname{KdV}(n, X)$ всех $I_{\pi}, \pi \in R(n, X)$, является бирациональным инбективным отображением. $В$ частности, $\operatorname{KdV}(n, X)$ расщепляется на несвязное обтединение образов соответствующих компактифииированных якобианов:

$$
\operatorname{KdV}(n, X)=\coprod_{\pi \in R(n, X)} I_{\pi}(W(\Gamma))
$$

1.13. ОПРЕДЕлЕНИЕ [38]. Пусть $\pi:(\Gamma, p) \rightarrow(X, q)$ - некоторое гиперэллиптическое касательное накрытие, $\mathscr{L} \in W(\Gamma)$ и $u(x):=u_{\mathscr{L}}(x)$ обозначает соответствуюший эллиптический конечно-зонньй потенциал (п. 1.9).

1) Назовем функцию $и(x)$ әллиптическим конечно-зонным потенциалом, ассоциированным с $(\pi ; \mathscr{L})$ (или с $\left.\left(\Gamma, p, \lambda_{\pi}, \mathscr{L}\right)\right)$.

2 ) Назовем функцию $u(x)$, определенную как мероморфная функция над $X=\mathbb{C} / \Lambda$, $u: X \rightarrow \mathbb{P}^{1}(\mathbb{C})$, непримитивной, если существует нетривиальная изогения $\varphi:(X, q)$ $\rightarrow\left(X^{\prime}, q^{\prime}\right)$, факторизуюшая $u$.

1.14. ЗАмЕчАния. 1) Пусть $u(x): X \rightarrow \mathbb{P}^{1}(\mathbb{C})$ - непримитивная функция как в п. 1.13.2) и $\widehat{\varphi}:\left(X^{\prime}, q^{\prime}\right) \rightarrow(X, q)$ обозначает дуальную изогению. Тогда $d:=$ $\operatorname{deg} \varphi=\operatorname{deg} \widehat{\varphi}$ делит $n, \widehat{\varphi}$ факторизует $\pi$ и обратньй морфизм, $\varphi^{*}: X_{n / d}^{\prime} \rightarrow X_{n}$, $\sum_{j} \beta_{j} \mapsto \sum_{j} \varphi^{*}\left(\beta_{j}\right)$, факторизует $I_{\pi}: W(\Gamma) \rightarrow X_{n}$. Более точно, существует гиперэллиптическое касательное накрытие $\pi^{\prime}:(\Gamma, p) \rightarrow\left(X^{\prime}, q^{\prime}\right)$ такое, что $n=d \cdot \operatorname{deg} \pi^{\prime}$, $\pi=\widehat{\varphi} \circ \pi^{\prime}$ и $I_{\pi}=\varphi^{*} \circ I_{\pi^{\prime}}$.

$2)$ Обратно, если $\pi:(\Gamma, p) \rightarrow(X, q)$ факторизуется изогенией степени $d>1$, $\psi:\left(X^{\prime}, q^{\prime}\right) \rightarrow(X, q)$, как $\pi=\psi \circ \pi^{\prime}$, то $n=d \cdot \operatorname{deg} \pi^{\prime}, \pi^{\prime}:(\Gamma, p) \rightarrow\left(X^{\prime}, q^{\prime}\right)$ является гиперэллиптическим касательным накрытием степени $n / d$ и $I_{\pi}$ факторизуется как $I_{\pi}=\varphi^{*} \circ I_{\pi^{\prime}}$, где $\varphi$ равно $\widehat{\psi}$, дуальной изогении $\psi$. Поэтому любой эллиптический конечно-зонньй потенциал, ассоциированный с $\pi$, является непримитивньм и факторизуется $\varphi=\widehat{\psi}: X \rightarrow X^{\prime}$. Будем также назьвать отображение $\pi$ непримитивны.м.

1.15. Основьваясь на данных вьше определениях и введенных понятиях, мы опишем структуру статьи и развиваемые далее методы для решения поставленной задачи.

1) Критерий касательности 1.5 является промежуточньм шагом на пути к использованию подхода алгебраических поверхностей в разделах 2 и 3 . Основную роль здесь играет раздутие некоторых линейчатых поверхностей $\pi_{S^{\perp}}: S^{\perp} \rightarrow S \rightarrow X$ и их факторов при действии естественной инволюции $\tau^{\perp}, S^{\sim}:=S^{\perp} / \sim_{\tau}$. Мы начинаем с доказательства того, что любое гиперэллиптическое касательное накрытие $\pi:(\Gamma, p) \rightarrow$ $(X, q)$ единственным образом факторизуется в результате действия $\pi_{S^{\perp}}$. Будем говорить, что $\pi$ имеет “тип” $\mu=\left(\mu_{i}\right)$, если образ $\Gamma$ в $S^{\perp}$ пересекает исключительньй дивизор $r_{i}^{\perp}$ с кратностью $\mu_{i}$ при $i=0, \ldots, 3$ (пा. 2.1, 2.2). Последнее означает, что (описательно говоря) $\Gamma$ имеет $\mu_{i}+\delta_{i 0}$ точек Вейерштрасса над полупериодами $\omega_{i}$ $(i=0, \ldots, 3)$. Для любых $\mu \in \mathbb{N}^{4}$ и $n \geqslant 1$ обозначим через $R(n, \mu, X)$ подмножество $R(n, X)$, образованное всеми накрытиями $\pi \in R(n, X)$, имеюшими “тип” $\mu$. Элементы $R(n, \mu, X)$ могут быть сопоставлены неприводимым дивизорам специальной линейной системы на $S^{\perp}$. Знание соответствующих классов эквивалентности позволяет в 
итоге получить основные результаты относительно $R(n, \mu, X)$. Например, если выполняются условия $\mu_{0}+1 \equiv \mu_{1} \equiv \mu_{2} \equiv \mu_{3} \equiv n(\bmod 2)$, но не выполняется условие $\left(2 n+1-\sum_{i=0}^{3} \mu_{i}^{2}\right) \in 4 \mathbb{N}$, то множество $R(n, \mu, X)$ пусто (п. 2.3). В противном случае $R(n, \mu, X)$, а также $R(n, X)$ конечны и любое $\pi \in R(n, \mu, X)$ имеет (арифметический) род $\frac{1}{2}\left(-1+\sum_{i=0}^{3} \mu_{i}\right)($ ㅍ․ 2.10, 2.6).

2) Поскольку естественная инволюция $\tau^{\perp}: S^{\perp} \rightarrow S^{\perp}$ индуцирует гиперэллиптическую инволюцию на любой из кривых $\Gamma$, то проекция $\Gamma$ в $S^{\sim}:=S^{\perp} / \sim_{\tau} \perp$ является рациональной неприводимой кривой из специальной линейной системы, например $|\lambda(n, \mu)|$, в $S^{\sim}$ (п. 3.1). Соответственно, любая неприводимая рациональная кривая в $|\lambda(n, \mu)|$ порождает элемент $R(n, \mu, X)$ (п. 2.6). Этот последний результат используется, в частности, при доказательстве сушествования некоторого бесконечного семейства минимально-гиперэллиптических касательных накрытий. А именно, мы начинаем с сопоставления исключительного дивизора $S^{\sim}$ каждому $\mu=\left(\mu_{i}\right) \in \mathbb{N}^{4}$, удовлетворяющему равенству $\mu_{0}+1 \equiv \mu_{1} \equiv \mu_{2} \equiv \mu_{3}(\bmod 2)$. Ограничение $\pi_{S^{\perp}}: S^{\perp} \rightarrow X$ на прообраз (при отображении $S^{\perp} \rightarrow S^{\sim}$ ) этого исключительного дивизора, как показывается далее, является минимально-гиперэллиптическим касательным накрытием типа $\mu$ и степени $n:=\frac{1}{2}\left(-1+\sum_{i=0}^{3} \mu_{i}^{2}\right)$. Таким образом, мы получаем единственньй элемент пространства модулей $R(n, \mu, X)$, назьваемьй далее исключительным накрытием типа $\mu$ ( и степени $\left.n:=\frac{1}{2}\left(-1+\sum_{i=0}^{3} \mu_{i}^{2}\right)\right)$ над $X$ (см. пп. 3.4, 4.1-4.4).

3) Оставшаяся часть раздела 4 посвяшается изучению (впервые!) новых случаев. Мы начинаем с доказательства того, что для всех $n \geqslant 1$ и $\mu \in \mathbb{N}^{4}$ таких, что

$$
\mu_{0}+1 \equiv \mu_{1} \equiv \mu_{2} \equiv \mu_{3} \quad(\bmod 2) \quad \text { и }\left(2 n+1-\sum_{i=0}^{3} \mu_{i}^{2}\right) \in 4 \mathbb{N}
$$

размерность линейной системы $|\lambda(n, \mu)|$ равна $\frac{1}{4}\left(2 n+1-\sum_{i=0}^{3} \mu_{i}^{2}\right)$ (п. 4.9). Отсюда следует, что если $\sum_{i=0}^{3} \mu_{i}^{2}=2 n-3$ (это первьй “неисключительньй случай”!), то множество $R(n, \mu, X)$ находится во взаимно однозначном соответствии с неприводимьми рациональными слоями пучка эллиптических кривых на $S \sim$. Применяя формулу Лефшеца (см., например, [23; с. 509]), связьваюшую характеристику Эйлера-Пуанкаре слоев пучка с $S^{\sim}$, нам удается вычислить размерность $R(n, \mu, X)$ (п. 4.12). Последнее подразумевает существование нового семейства гиперэллиптических касательных накрытий (отличного от семейства исключительных накрытий).

4) В разделе 5 и далее мы сопоставляем каждому гиперэллиптическому касательному накрытию степени $n$ и арифметического рода $g$, например $\pi:(\Gamma, p) \rightarrow(X, q)$, следуюшие восемь “канонических" $\theta$-характеристик (п. 5.3.3)):

$$
\left\{\begin{array}{l}
\xi=\mathscr{O}_{\Gamma}\left((g-1) p+\pi^{*}\left(\omega_{i}-q\right)\right), \\
\xi=\mathscr{O}_{\Gamma}\left((g-1-n) p+\pi^{*}\left(\omega_{i}\right)\right)
\end{array} \quad(i=0, \ldots, 3),\right.
$$

где, как обычно, $\left\{\omega_{i}, i=0, \ldots, 3\right\}\left(\omega_{0}=q\right)$ обозначает множество $\frac{1}{2} \Lambda / \Lambda$, полупериодов $(X, q)$. Нахождение соответствующих четных эллиптических конечно-зонных потенциалов (см. п. 1.9) сводится к вычислению пересечений их $X$-орбит $\left(\operatorname{Orb}_{\xi} X\right)$ с тэта-дивизором $\Theta_{\Gamma}$. Поскольку мы рассматриваем (гиперэллиптические) касательные накрытия, $\operatorname{Orb}_{\xi}(X)$ является также первым $K P$-потоком на $W(\Gamma)$, ассоциированным с данными $(\Gamma, p, \xi)$. Аналогично, мы можем вычислить кратность его пересечения с $\Theta_{\Gamma}$ 
в произвольной точке с помощью формулы Фея-Сигала-Вильсона раздела 5 ([15], [33], см. также $[41 ; \S 3])$. Эта задача была полностью решена для исключительных накрытий, как это объясняется в оставшейся части раздела 5. Напомним, что для любого исключительного накрытия типа $\mu$ четньй эллиптический конечно-зонньй потенциал, ассоциированньй с введенной вьше “канонической” $\theta$-характеристикой, равен

$$
u_{\xi}(x)=\sum_{i=0}^{3} a_{i}\left(a_{i}+1\right) \wp\left(x-\omega_{i}\right)
$$

для некоторых $\left(a_{i}\right) \in \mathbb{N}^{4}$, выражаемых, в свою очередь, через $\left(\mu_{i}\right)$ (см., например, п. 5.5) и таких, что $2 n=\sum_{i=0}^{3} a_{i}\left(a_{i}+1\right)$. Обратно, для данного целочисленного вектора $\left(a_{i}\right) \in \mathbb{N}^{4}$ сушествует единственньй вектор $\mu$ такой, что

$$
u(x)=\sum_{i=0}^{3} a_{i}\left(a_{i}+1\right) \wp\left(x-\omega_{i}\right)
$$

является эллиптическим конечно-зонным потенциалом, ассоциированным с одной из “канонических" $\theta$-характеристик над исключительньм накрытием (типа $\mu$ ), степени

$$
n:=\frac{1}{2} \sum_{i=0}^{3} a_{i}\left(a_{i}+1\right)
$$

и арифметического рода

$$
\frac{1}{2} \max \left\{2 M, 1+\Sigma-\left(1+(-1)^{\Sigma}\right)\left(m+\frac{1}{2}\right)\right\},
$$

где

$$
M:=\max \left\{a_{i}\right\}, \quad \sum:=\sum_{i=0}^{3} a_{i} \text { и } m:=\min \left\{a_{i}\right\}
$$

(см. также [36]).

5) Поскольку нашей целью является перенесение этих результатов на случаи, отличные от исключительных накрытий, мы производим (начиная с раздела 6) аналогичные рассмотрения следующего интересного случая: $\bigcup_{2 n-3=\Sigma \mu_{i}^{2}} R(n, \mu, X)$. Мы разрешаем проблему характеризации “канонически" ассоциированных эллиптических конечно-зонных потенциалов путем глобализации нашей предыдущей конструкции. Пусть $\mathfrak{X}$ обозначает пространство модулей эллиптических кривых, характеризуемых их полупериодами, и пусть $\mathscr{R}(n, \mu)$ обозначает алгебраическое семейство $\bigcup_{\mathfrak{X}} R(n, \mu, X)$. Предположим (в разделе 6 и далее), что $\mu^{(2)}=2 n-3$, и сопоставим каждому $\pi \in \mathscr{R}(n, \mu)$ одну из канонических $\theta$-характеристик, $\pi \mapsto \xi$ (например, $\left.\xi=\mathscr{O}_{\Gamma}((g-1) p)\right)$. Соответствуюший эллиптический конечно-зонный потенщиал, $u_{\xi}(x)$, равен в этом случае

$$
u_{\xi}(x) \equiv 2 \wp(x-\rho)+2 \wp(x+\rho)+\sum_{i=0}^{3} a_{i}\left(a_{i}+1\right) \wp\left(x-\omega_{i}\right)
$$


при некотором $\vec{a}=\left(a_{i}\right) \in \mathbb{N}^{4}$ таком, что $n=2+\frac{1}{2} \sum_{i=0}^{3} a_{i}\left(a_{i}+1\right)$ (раздел 6$)$, и $\rho$ из $X$, $2 \rho \neq 0$, удовлетворяюшем уравнению

$$
\sum_{i=0}^{3}\left(2 a_{i}+1\right)^{2} \wp^{\prime}\left(\rho-\omega_{i}\right)=0 .
$$

6) Уравнение (7), действительно, является необходимым и достаточным условием того, что такая функция является эллиптическим конечно-зонным потенциалом (п. 6.2). Мы также приходим к выводу, что $\rho \mapsto\left(\wp(\rho): \wp\left(\omega_{1}\right): \wp\left(\omega_{2}\right)\right) \in \mathbb{P}^{2}(\mathbb{C})$ определяет изоморфизм между множеством решений

$$
\left\{\sum_{i=0}^{3}\left(2 a_{i}+1\right)^{2} \wp^{\prime}\left(\rho-\omega_{i}\right)=0, \rho \in X, X \in \mathfrak{X}\right\}
$$

и афффинной секстикой $\mathscr{C}_{\vec{a}} \backslash\left\{O_{1}, O_{2}, O_{3}\right\}$ (пा. 7.3, 7.4). Наконец, мы доказьваем, что композиция последних отображений

$$
\pi \mapsto \xi \mapsto u_{\xi}(x) \mapsto\left(\wp(\rho): \wp\left(\omega_{1}\right): \wp\left(\omega_{2}\right)\right)
$$

приводит к собственно инъективному отображению (а именно вложению) $\mathscr{R}(n, \mu)$ в $\mathscr{C}_{\vec{a}} \backslash\left\{O_{1}, O_{2}, O_{3}\right\}$ (п. 7.7, см. также п. 9.6).

7) Обратно, для любого $X \in \mathfrak{X}$ и любого решения уравнения (7) (эквивалентно, для любой точки $\left.\mathscr{C}_{\vec{a}} \backslash\left\{O_{1}, O_{2}, O_{3}\right\}\right)$ естественно поставить вопрос, является ли функшия (6) эллиптическим конечно-зонным потенциалом, и найти соответствуюшие спектральные данные $(\pi ; \xi)$ при утвердительном ответе на этот вопрос. Например, если $\mathscr{C}_{\vec{a}}$ неприводимо, то $\mathscr{R}(n, \mu)$ накрывает всю аффинную секстику $\mathscr{C}_{\vec{a}} \backslash\left\{O_{1}, O_{2}, O_{3}\right\}$, и любая такая функция $u(x)$ - эллиптический конечно-зонный потенциал, ассоциированньй с хорошо определенной канонической $\theta$-характеристикой над некоторьм $\pi \in$ $R(n, \mu, X)$. Вообще говоря, последнее утверждение остается верным для любой функции $u(x)$, описанной вьше, если и только если $\left(\wp(\rho): \wp\left(\omega_{1}\right): \wp\left(\omega_{2}\right)\right)$ принадлежит к образу $\mathscr{R}(n, \mu)$. Следовательно, мы естественно находим целочисленные векторы $\vec{a}=\left(a_{i}\right) \in \mathbb{N}^{4}$, для которых $\mathscr{C}_{\vec{a}}$ приводимо, что позволяет предъявить соответствующее разложение. Примечательно, что последняя задача снова сводится в разделе 8 к изучению приводимых слоев пучка эллиптических кривых (т.е. образованных секстиками $\mathscr{C}_{\vec{a}}$ и $\left.\mathscr{C}_{0}\right)$ и применению той же формулы Лефшеца, как и в разделе 4.

8) Собственно говоря, дополнение образа $\mathscr{R}(n, \mu)$ в $\mathscr{C}_{\vec{a}} \backslash\left\{O_{1}, O_{2}, O_{3}\right\}$ также параметризует эллиптические конечно-зонные потенщиалы, хотя и другого типа. Последние потенциалы соответствуют ( “исключительньм") гиперэллиптическим касательным накрытиям степени $n$, но типа $\mu^{\prime}$, отличного от $\mu$ (пш. 9.4, 9.6.4) и 9.9). Наш подход “эллиптических поверхностей” позволяет в итоге дать (см. конец раздела 9) достаточно исчерпьвающий ответ на наш предыдущий вопрос: для любого $\vec{a}=\left(a_{i}\right) \in \mathbb{N}^{4}$, любой эллиптической кривой $X \in \mathfrak{X}$ и произвольного $\rho \in X \backslash\left\{\omega_{i}\right\}$ функция (6) есть эллиптический конечно-зонньй потенщиал тогда и только тогда, когда вьполняется условие (7). Подробнее, пусть $g$ обозначает натуральное число

$$
g:=\frac{1}{2} \max \left\{2 M, 1+\Sigma-\left(1+(-1)^{\Sigma}\right)\left(m+\frac{1}{2}\right)\right\}
$$


где

$$
M:=\max \left\{a_{i}\right\}, \quad \Sigma:=\sum_{i=0}^{3} a_{i} \text { и } m:=\min \left\{a_{i}\right\},
$$

и пусть $\Pi_{\vec{a}}(x, y, z)$ - однородные многочлены, определяемые в п. 9.9. Тогда если выполняется $(7)$, но $\Pi_{\vec{a}}\left(\wp(\rho), \wp\left(\omega_{1}\right), \wp\left(\omega_{2}\right)\right) \neq 0$, эллиптический конечно-зонньй потенциал $u(x)$ ассоциирован с канонической $\theta$-характеристикой над гиперэллиптическим касательным накрытием степени

$$
n:=2+\frac{1}{2} \sum_{i=0}^{3} a_{i}\left(a_{i}+1\right)
$$

рода $g$ и типа $\mu=\left(\mu_{i}\right)$ такого, что $\sum_{i=0}^{3} \mu_{i}^{2}=2 n-3$. В другом случае, т.е. если вьполняется $(7)$ и $\Pi_{\vec{a}}\left(\wp(\rho), \wp\left(\omega_{1}\right), \wp\left(\omega_{2}\right)\right)=0$, потенщиал $u(x)$ ассоциирован с "не канонической" $\theta$-характеристикой над исключительным накрытием такой же степени $n$, рода $g+1$ и типа $\widehat{\mu}=\left(\widehat{\mu}_{i}\right)$ такого, что $\sum_{i=0}^{3} \widehat{\mu}_{i}^{2}=2 n+1$ (более подробное описание см. в п. 9.9).

9) Заключим этот длинньй вводньй раздел формулированием некоторой открытой проблемы. Для любой эллиптической кривой $X \in \mathfrak{X}$, произвольного $\mu=\left(\mu_{i}\right)$ (как в п. 1.15.4)) и любого $n \in \mathbb{N}$ такого, что $\sum_{i=0}^{3} \mu_{i}^{2}=2 n+1$, множество $\mathscr{R}(n, \mu, X)$ содержит единственньй элемент - “исключительное накрытие типа $\mu$ ” над $X$. В $[18$; $\S 3.5$. iii)] было доказано, что для “достаточно общей” $X$ исключительное накрытие типа $\mu$ над $X$ является гладким. В случае $\sum_{i=0}^{3} \mu_{i}^{2}=2 n-3$ (см. п. 2.3) множество $\mathscr{R}(n, \mu, X)$ является конечным и для обшей эллиптической кривой $X_{\text {gen }}$ его размер-

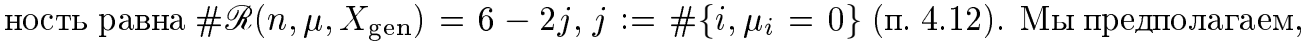
что соответствующее накрытие также является гладким.

\section{2. Гиперэллиптические касательные накрытия как дивизоры проективной поверхности $S^{\perp}[38]$}

2.1. Пусть $\mathscr{E}$ - единственньй (с точностью до изоморфизма) неразложимьй локально свободньй пучок ранга 2 и степени 0 над эллиптической кривой $(X, q)$. Пусть $S=\mathbb{P}(\mathscr{E})$ обозначает ассоциированное расслоение проективного пространства, канонически снабженное проекцией $\pi_{S}: S \rightarrow X$. Хорошо известно, что $S$ - единственное линейчатое пространство над $X$, имеющее только одно сечение (обозначаемое далее $\left.C_{0}\right)$ самопересечений 0 . Обратный гомеоморфизм $[-1]:(X, q) \rightarrow(X, q)$ может быть поднят как инволющия $S, \tau: S \rightarrow S$, с двумя неподвижными точками над каждым из полупериодов, $\omega_{i}(i=0, \ldots, 3)$, кривой $(X, q)$ : однав $C_{0}$, обозначаемая $s_{i}$, и другая, обозначаемая $r_{i}$. Пусть $e: S^{\perp} \rightarrow S$ обозначает раздутие $S$ в восьми неподвижных точ-

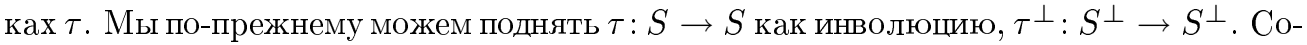
ответствующие исключительные дивизоры, обозначаемые как $\left\{r_{i}^{\perp}, s_{i}^{\perp}, i=0, \ldots, 3\right\}$, остаются неподвижными при действии $\tau^{\perp}$, и фактор $S^{\sim}:=S^{\perp} / \sim_{\tau} \perp$ является гладкой рациональной поверхностью. Степень 2 проекции $\phi: S^{\perp} \rightarrow S^{\sim}$ непосредственно связана с множеством всех гиперэллиптических касательных накрытий. На самом деле, как это будет показано далее, каждое такое накрытие единственным образом факторизуется посредством $\pi_{S^{\perp}}:=\pi_{S} \circ e: S^{\perp} \rightarrow S$ и может быть восстановлено по численно описанной рациональной неприводимой кривой в $S^{\sim}$ (п. 2.6). 
2.2. ОПрЕДЕлЕнИЕ. Пусть $\pi:(\Gamma, p) \rightarrow(X, q)$ - гиперэллиптическое касательное накрытие. Обозначим $i^{\perp}: \Gamma \rightarrow S^{\perp}$ единственньй морфизм такой, что $\pi=\pi_{S} \circ e \circ i^{\perp}$. Назовем типом $\pi$ кратность пересечения вектора $\mu=\left(\mu_{i}\right):=\left(i^{\perp}(\Gamma) \cdot r_{i}^{\perp}\right) \in \mathbb{N}^{4}$ и обозначим через $\mu^{(1)}$ и $\mu^{(2)}$ соответственно суммы $\mu^{(1)}:=\sum_{i=0}^{3} \mu_{i}$ и $\mu^{(2)}:=\sum_{i=0}^{3} \mu_{i}^{2}$.

2.3. ПРЕДЛОЖЕНИЕ. Пусть $\pi:(\Gamma, p) \rightarrow(X, q)$ - әиперәллиптическое касательное накрытие степени $n$ и типа $\mu$ u $i^{\perp}: \Gamma \rightarrow S^{\perp}-$ каноническое разложение $\pi$ по $е: S^{\perp} \rightarrow S$. Тогда

1) образ $\Gamma^{\perp}:=i^{\perp}(\Gamma)$ является $\tau^{\perp}-$ инвариантным и линейно эквивалентнысм⿻ $e^{*}\left(n C_{0}+S_{0}\right)-s_{0}^{\perp}-\sum_{0}^{3} \mu_{i} r_{i}^{\perp}$, zде $S_{0}=\pi_{S}^{*}(q) ;$

2) ограничение $\tau^{\perp}$ на $\Gamma^{\perp}$ индуцирует гиперэллиптическую инволючию на $\Gamma$ и $\phi\left(\Gamma^{\perp}\right)-$ рачиональная неприводимая кривая в $S^{\sim}$. При этом имеют место следующие (не)равенства между $n, \mu^{(1)}, \mu^{(2)}$ и арифметическим родом $g$ кривой $\Gamma$ :

3) $\mu_{0}+1 \equiv \mu_{1} \equiv \mu_{2} \equiv \mu_{3} \equiv n(\bmod 2)$;

4) $\mu^{(2)} \leqslant 2 n+1 u\left(2 n+1-\mu^{(2)}\right) \in 4 \mathbb{N}$;

5) $2 g+1 \leqslant \mu^{(1)} u \frac{1}{2} g(g+1) \leqslant n$.

2.4. ОПРЕДЕЛЕНИЕ. Мы будем назьвать целочисленньй вектор $\mu \in \mathbb{N}^{4} n$-noдходящим (при $n \in \mathbb{N}),{ }^{1}$ если $\mu_{0}+1 \equiv \mu_{1} \equiv \mu_{2} \equiv \mu_{3}(\equiv n)(\bmod 2)$.

2.5. ЛЕммА. Для любой пары $(n, \mu) \in \mathbb{N} \times \mathbb{N}^{4}$ такой, что $\mu-n$-подходящий вектор, существует единственный класс эквивалентности в $\operatorname{Pic}\left(S^{\sim}\right)$, обозначаемый $\lambda(n, \mu)$, такой, что

$$
\phi^{*}(\lambda(n, \mu)) \equiv e^{*}\left(n C_{0}+S_{0}\right)-s_{0}^{\perp}-\sum_{0 \leqslant i \leqslant 3} \mu_{i} r_{i}^{\perp} .
$$

2.6. ТЕОРемА. Для любых $(n, \mu)$ таких, как в $n .2 .4$, предположим, что $C^{\sim}$ - рациональная неприводимая кривая в $|\lambda(n, \mu)|, j: \mathbb{P}^{1}(\mathbb{C}) \rightarrow C^{\sim}-$ eе десингуляризачия, и рассмотрим произведение слоев, $\widehat{\Gamma}:=\mathbb{P}^{1}(\mathbb{C}) \times_{C^{\sim}} \phi^{*}\left(C^{\sim}\right)$, отображения $j$ и ограничение $\phi: S^{\perp} \rightarrow S^{\sim}$ на $\phi^{*}\left(C^{\sim}\right)$. Тогда первая проекчия $\widehat{\Gamma} \rightarrow \mathbb{P}^{1}(\mathbb{C})$ определяет гиперэллиптическую инволющию на $\widehat{\Gamma}$, оставляющую неподвижной гладкую точку $p:=\widehat{\Gamma} \cap s_{0}^{\perp}$, в то время как композиция второй проекции $\widehat{\Gamma} \rightarrow \phi^{*}\left(C^{\sim}\right)$ с $\pi_{S^{\perp}}: S^{\perp} \rightarrow X$ является минимально-гиперэллиптическим касательным накрытием, $\widehat{\pi}:(\widehat{\Gamma}, p) \rightarrow(X, q)$, степени $n$, типа $\mu$ и арифметического рода $\widehat{g}:=\frac{1}{2}\left(\mu^{(1)}-1\right)$.

2.7. ЗАмЕчАНИЯ. 1$)$ Пусть $j:(\Gamma, p) \rightarrow(\widehat{\Gamma}, p)$ - некоторьй отмеченный морфизм степени 1. Тогда $\pi:=\widehat{\pi} \circ j:(\Gamma, p) \rightarrow(X, q)$ также является гиперэллиптическим касательным накрытием степени $n$ и типа $\mu$, но арифметический род меньше или равен $\frac{1}{2}\left(\mu^{(1)}-1\right)$ (равенство достигается, если $j-$ изоморфизм).

2) Обратно, для любого гиперэллиптического касательного накрытия $\pi:(\Gamma, p) \rightarrow$ $(X, q)$ степени $n$ и типа $\mu$ его канонический образ в $S^{\sim}, \phi \circ i^{\perp}(\Gamma)$, является неприводимой рациональной кривой в $|\lambda(n, \mu)|$. Причем если $\widehat{\pi}:(\widehat{\Gamma}, p) \rightarrow(X, q)$ - гиперэллиптическое касательное накрытие (степени $n$, типа $\mu$ и арифметического рода $\widehat{g}:=$ $\left.\frac{1}{2}\left(\mu^{(1)}-1\right)\right)$, канонически ассоциированное с $\phi \circ i^{\perp}(\Gamma)$, то $\pi$, очевидно, факторизуется посредством $\widehat{\pi}$ и $\widehat{\pi}$ - минимально-гиперэллиптическое накрытие (п. 1.11).

\footnotetext{
${ }^{1}$ В оригинале "fits in (with $n \in \mathbb{N}$ )". - Прим. перев.
} 
2.8. ОПРЕДЕлЕНИЕ. Для любой пары $(n, \mu)$ такой, как в п. 2.4 , обозначим через $R(n, \mu, X)$ множество классов эквивалентности гиперэллиптических касательных накрытий степени $n$, типа $\mu$ и арифметического рода $g:=\frac{1}{2}\left(\mu^{(1)}-1\right)$.

2.9. ЗАмЕчАния. 1) В соответствии с п. 2.7, каждое гиперэллиптическое касательное накрытие степени $n$ и типа $\mu$ изоморфно частичной десингуляризации единственного элемента $R(n, \mu, X)$. В частности, $\pi \in R(n, \mu, X)$ тогда и только тогда, когда $\pi$ является минимально-гиперэллиптическим касательным накрытием степени $n$ и типа $\mu$.

2) Для любого гиперэллиптического касательного накрытия, $\pi:(\Gamma, p) \rightarrow(X, q)$, степени $n$ и типа $\mu$ арифметический род кривой $Г$ меньше или равен $\frac{1}{2}\left(\mu^{(1)}-1\right)$, причем равенство достигается только при $\pi \in R(n, \mu, X)$.

$3)$ Мы, в результате, узнали из п. 2.6, что сушествует взаимно однозначное соответствие между множеством $R(n, \mu, X)$ и множеством рациональных неприводимых кривых в $S^{\sim}$, линейно эквивалентных $\lambda(n, \mu)$.

4) На самом деле для любой рациональной кривой $C^{\sim} \in|\lambda(n, \mu)|$ соответствующий нормируюший морфизм $j: \mathbb{P}^{1}(\mathbb{C}) \rightarrow C^{\sim} \subset S^{\sim}$ не может быть деформирован $[38 ; § 4.10]$, что подразумевает следующий результат.

2.10. ТЕОРемА. Для любой эллиптической кривой $(X, q)$ и любой пары $(n, \mu) \in$ $\mathbb{N} \times \mathbb{N}^{4}$ таких, что вектор $\mu$ является $n$-подходящим, множсество $R(n, \mu, X)$, определенное в $n .2 .8$, конечно (но может оказаться и пустым).

\section{3. О счетности множества исключительных дивизоров на рациональных поверхностях $S \sim$ [38]}

Выберем некоторый слой $\ell$ расслоения $\pi_{S}: S \rightarrow X$ над дополнением к $(X, q)_{2}=$ $\left\{\omega_{i}\right\}$, и пусть $r_{i}^{\sim}$ и $s_{i}^{\sim}(i=0, \ldots, 3)$ - редуцированные образы $r_{i}^{\perp}$ и $s_{i}^{\perp}$ в $S^{\sim}$. Для любого дивизора $D$ в $S$ обозначим через $D^{\perp}\left(\subset S^{\perp}\right)$ его отображение при действии $e: S^{\perp} \rightarrow S$ и через $D^{\sim}$ - редуцированньй образ $D^{\perp}$ в $S^{\sim}, D^{\sim}:=\phi\left(D^{\perp}\right)$ (п. 2.1).

3.1. ЛеммА. 1) Поверхность $S^{\sim}$ рачиональна.

2) Группа $\operatorname{Pic}\left(S^{\sim}\right)$ - свободная группа ранга 10, и 10 дивизоров

$$
\left\{C_{0}^{\sim}, \ell^{\sim}, S_{i}^{\sim}=\pi_{S}^{*}\left(\omega_{i}\right)^{\sim}, r_{i}^{\sim}, i=0, \ldots, 3\right\}
$$

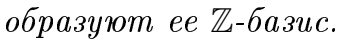

3) Для любых $i=0, \ldots, 3$ кривая $\ell^{\sim}$ линейно (и численно) эквивалентна $\ell^{\sim} \equiv$ $2 S_{i}^{\sim}+r_{i}^{\sim}+s_{i}^{\sim}$.

4) Для любы $n \geqslant 1$ и $n$-подходящих $\mu \in \mathbb{N}^{4}$ класс линейной эквивалентности $\lambda(n, \mu)$, определенной в $n .2 .5$, содерэит дивизор

$$
n\left(C_{0}^{\sim}+2 \ell^{\sim}\right)-(n-1) S_{0}^{\sim}-\frac{1}{2}\left(n+\mu_{0}-1\right) r_{0}^{\sim}-\sum_{1 \leqslant j \leqslant 3}\left\{n S_{j}^{\sim}+\frac{1}{2}\left(n+\mu_{j}\right) r_{j}^{\sim}\right\} .
$$

5) Для любой пары $(n, \mu)$ из $n$. 3.1.4) и любого дивизора $D \in|\lambda(n, \mu)|$ существуют число $m \geqslant 0$, челочисленный $m$-подходящий вектор $\left(\nu_{i}\right)=\nu \in \mathbb{N}^{4}$ и неприводимая кривая $C^{\sim}$ такие, что:

- $C^{\sim} \in|\lambda(m, \nu)| ;$ 
- $\mu_{i} \leqslant \nu_{i} \partial \Omega_{я} 0 \leqslant i \leqslant 3$

- $m \leqslant n u m \equiv n(\bmod 2)$;

- $\mu^{(2)} \leqslant \nu^{(2)} \leqslant 2 m+1 \leqslant 2 n+1$

- D представляется в виде

$$
D=C^{\sim}+(n-m) C_{0}^{\sim}+\frac{1}{2} \sum_{0 \leqslant i \leqslant 3}\left\{(n-m) s_{i}^{\sim}+\left(\nu_{i}-\mu_{i}\right) r_{i}^{\sim}\right\} .
$$

3.2. ЗАМЕЧАНИЕ. Легко вьвести из П. 3.1.5), что для непустоты $|\lambda(n, \mu)|$ необходимо наложить условие $\mu^{(2)} \leqslant 2 n+1$. Мы вьчислим далее размерность $|\lambda(n, \mu)|$ и, в частности, приведем числовой критерий того, что цельй дивизор $S \sim$ принадлежит $|\lambda(n, \mu)|$.

3.3. ЛеммА. Пусть $K, K^{\perp}$ и $K^{\sim}$ - канонические дивизоры в $S, S^{\perp}$ и $S^{\sim}$ соответственно. Выберем такую пару $(n, \mu) \in \mathbb{N} \times \mathbb{N}^{4}$, что $\mu-n$-подходящий вектор (см. п. 2.4). Тогда

1) оба дивизора $e^{*}(K)$ и $\phi^{*}\left(K^{\sim}\right)$ линейно әквивалентны $K^{\perp}-\sum_{0 \leqslant i \leqslant 3}\left(s_{i}^{\perp}+r_{i}^{\perp}\right)$;

2) $K^{\sim} \cdot K^{\sim}=0, \lambda(n, \mu) \cdot K^{\sim}=-1 u \lambda(n, \mu)^{2}=\frac{1}{2}\left(2 n-1-\mu^{(2)}\right)$;

3) любой неприводимый дивизор $D \in|\lambda(n, \mu)|$ удовлетворяет равенству $D \cdot K^{\sim}$ $=-1$ (кроме того, $D \cdot \ell^{\sim}=n, D \cdot r_{i}^{\sim}=\mu_{i}$ для $\left.i=0, \ldots, 3\right)$, пересекает $s_{0}^{\sim} u$ его арифметический род равен $p_{a}(D):=\frac{1}{4}\left(2 n+1-\mu^{(2)}\right)$. Обратно, для любого неприводимого дивизора $D \subset S^{\sim}$, удовлетворяющего равенству $D \cdot K^{\sim}=-1 u$ пересекающего $s_{0}^{\sim}$, положим $m:=D \cdot \ell^{\sim} u \nu_{i}:=D \cdot r_{i}^{\sim}($ дя любьх $i=0, \ldots, 3)$. Тогда $m$ - натуральное число, вектор $\nu=\left(\nu_{i}\right) \in \mathbb{N}^{4}$ является $m$-подходящим $и$ $D \in|\lambda(m, \nu)|$.

3.4. ПРЕДЛОЖЕНИЕ. Пусть $(n, \mu) \in \mathbb{N} \times \mathbb{N}^{4}$ - произвольная пара такая, что $\mu$ является $n$-подходящим. Тогда проективное пространство $|\lambda(n, \mu)|$ пусто, если $2 n+1<\mu^{(2)}$, и имеет размерность $\operatorname{dim}|\lambda(n, \mu)|=\frac{1}{4}\left(2 n+1-\mu^{(2)}\right)$ в противном случае. При этом если $2 n+1 \geqslant \mu^{(2)}$, то әлемент общего положения в $|\lambda(n, \mu)|$ является гладкой неприводимой кривой рода $\frac{1}{4}\left(2 n+1-\mu^{(2)}\right)$.

3.5. СЛЕДСтВИЕ. Существует взаимно однозначное соответствие между множсеством $\bigcup_{(n, \mu)} R(n, \mu, X)$ (классов изоморфизмов минимально-гиперәллиптических касательных накрытий над $(X, q))$ и множсеством рачиональных кривых $C^{\sim}{ }_{\boldsymbol{\theta}} S^{\sim}$, пересекаюших $s_{0}^{\sim}$ u $\ell^{\sim}$ и таких, что $C^{\sim} \cdot K^{\sim}=-1$.

\section{4. Исключительные накрытия; другие случаи}

4.1. Напомним, прежде всего, что под исключительньм накрытием гладкой проективной поверхности мы понимаем неприводимый дивизор $D$, изоморфный $\mathbb{P}^{1}(\mathbb{C})$ и такой, что $D \cdot D=-1$ в самопересечениях. Выберем некоторьй вектор $\mu \in \mathbb{N}^{4}$ такой, что $\mu_{0}+1 \equiv \mu_{1} \equiv \mu_{2} \equiv \mu_{3}(\bmod 2)$. Тогда $2 n+1:=\mu^{(2)}$-нечетное число, $\mu$ является $n$-подходящим, $\operatorname{dim}|\lambda(n, \mu)|=0$ и единственный эффективньй дивизор в $|\lambda(n, \mu)|-$ исключительная кривая $S^{\sim}$, обозначаемая далее $C_{\mu}^{\sim}$. Эти исключительные кривые могут быть исчерпьвающе охарактеризованы как так назьваемые исключительные (гиперэллиптические касательные) накрытия. 
4.2. ТЕОРема [38; $§ 5]$. Пусть вектор $\mu \in \mathbb{N}^{4}$ такой, как в $n .4 .1, n:=\frac{1}{2}\left(\mu^{(2)}-1\right)$, и пусть $C_{\mu}^{\sim}$ обозначает единственную исключительную кривую в $S^{\sim}$, линейно әквивалентную $\lambda(n, \mu)$. Тогда $C_{\mu}^{\sim}$ пересекает $s_{0}^{\sim}$ и $\ell^{\sim}$ и выполняются следующие равенства: $C_{\mu}^{\sim} \cdot s_{0}^{\sim}=1, C_{\mu}^{\sim} \cdot \ell^{\sim}=n, C_{\mu}^{\sim} \cdot r_{i}^{\sim}=\mu_{i}, i=0, \ldots, 3$.

Обратно, пусть дана некоторая исключительная кривая $C^{\sim}$ на $S^{\sim} ;$ если $C^{\sim}$ пересекает $s_{0}^{\sim} u \ell^{\sim}$, то $C^{\sim} \cdot s_{0}^{\sim}=1$.

Кроме того, иелочисленный вектор $\nu=\left(\nu_{i}\right)=\left(C^{\sim} \cdot r_{i}^{\sim}\right)-m$-подходящий при $m:=C^{\sim} \cdot \ell^{\sim}, \nu^{(2)}=2 m+1 u C^{\sim}=C_{\nu}^{\sim}$.

4.3. ОПРЕДЕЛЕНИЕ $[38 ; \S 6.2]$. Пусть $\mu \in \mathbb{N}^{4}$ и $C_{\mu}^{\sim}$ такие, как в п. 4.2. Рассмотрим кривую $C_{\mu}^{\perp}:=\phi^{*}\left(C_{\mu}^{\sim}\right) \subset S^{\perp}$, снабженную гладкой точкой $p_{\mu}:=C_{\mu}^{\perp} \cap s_{0}^{\perp}=$ $\phi^{-1}\left(C_{\mu}^{\sim} \cap s_{0}^{\sim}\right)$. Тогда отмеченная проекция $\pi_{\mu}:\left(C_{\mu}^{\perp}, p_{\mu}\right) \rightarrow(X, q)$, получаемая ограничением $\pi_{S \perp}: S^{\perp} \rightarrow X$ на $C_{\mu}^{\perp}$, является минимально-гиперэллиптическим касательным накрытием типа $\mu$, степени $n:=\frac{1}{2}\left(\mu^{(2)}-1\right)$ и арифметического рода $=\frac{1}{2}\left(\mu^{(1)}-1\right)$ (ср. с п. 2.6). Будем назьвать $\pi_{\mu}$ исключительным накрытием типа $\mu$ над $(X, q)$.

4.4. Теорема. Пусть $\pi:(\Gamma, p) \rightarrow(X, q)$ - гиперәллиптическое касательное накрытие степени $n$ и типа $\mu$. Следующие свойства әквивалентны при условии выполнения равенства $2 n+1=\mu^{(2)}($ ср. с [38; §6.1]):

i) канонический морфизм $i^{\perp}: \Gamma \rightarrow S^{\perp}$ является вложением;

ii) $\pi$ - минимально-гиперәллиптическое накрытие и $\phi\left(i^{\perp}(\Gamma)\right)$ - исключительная кривая в $S^{\sim}$;

iii) арифметический род Г равен $\frac{1}{2}\left(\mu^{(1)}-1\right)$ и $\phi\left(i^{\perp}(\Gamma)\right)=C_{\mu}^{\sim}$;

iv) $\pi$ изоморфно исключительному накрытию $\pi_{\mu}$.

4.5. ОПРЕДЕЛЕНИЕ. Введем на множестве $\left\{(n, \mu) \in \mathbb{N} \times \mathbb{N}^{4}, \mu-n\right.$-подходящий вектор и $\left.\mu^{(2)} \leqslant 2 n+1\right\}$ следуюшее частичное упорядочение: $(m, \nu)$ меньше $(n, \mu)$ (обозначение: $(m, \nu) \prec(n, \mu))$ тогда и только тогда, когда $n-m \in 2 \mathbb{N}$ и $\nu-\mu \in 2 \mathbb{N}^{4}$. В последнем случае имеет место следуюшее разложение:

$$
\lambda(n, \mu) \equiv \lambda(m, \nu)+(n-m) C_{0}^{\sim}+\frac{n-m}{2} \sum_{0 \leqslant i \leqslant 3} s_{i}^{\sim}+\frac{1}{2} \sum_{0 \leqslant i \leqslant 3}\left(\nu_{i}-\mu_{i}\right) r_{i}^{\sim}
$$

$\mathrm{K}$ тому же эффективный дивизор $\lambda(n, \mu)-\lambda(m, \nu)$ определяет инъективное отображение $|\lambda(m, \nu)|$ как проективное подпространство в $|\lambda(n, \mu)|$. Мы будем отождествлять $|\lambda(m, \nu)|$ с его образом в $|\lambda(n, \mu)|$ (всякий раз, когда $(m, \nu) \prec(n, \mu))$.

4.6. ЛЕмма [38; $§ 5.2]$. Пусть пара $(n, \mu) \in \mathbb{N} \times \mathbb{N}^{4}$ такова, ито н есть $n$-подходящий вектор и $\mu^{(2)} \leqslant 2 n+1$, и пусть $D \subset S^{\sim}-$ некоторый әффективный дивизор, линейно әквивалентный $\lambda(n, \mu)$. Тогда $D$ является приводимым тогда и только тогда, когда существуют такие $(m, \nu) \prec(n, \mu)((m, \nu) \neq(n, \mu))$, что $D \in|\lambda(m, \nu)| \subset|\lambda(n, \mu)|$.

ДокаЗАТЕльство. Действительно, $D$ приводим тогда и только тогда, когда приводим $\tau^{\perp}$-инвариантньй дивизор $S^{\perp}, \phi^{*}(D)$. С другой стороны, $e_{*}\left(\phi^{*}(D)\right)$ есть $\tau$-инвариантньй дивизор $S$, линейно эквивалентньй $n C_{0}+S_{0}$. Отсюда следует сушествование такого $m \leqslant n, m \equiv n(\bmod 2)$, и такого $\tau$-инвариантного дивизора 
$\Gamma \in\left|m C_{0}+S_{0}\right|$, что $e_{*}\left(\phi^{*}(D)\right)$ раскладьвается как $\Gamma+(n-m) C_{0}$. Мы можем восстановить $\phi^{*}(D)$ по $\Gamma+(n-m) C_{0}$ :

$$
\phi^{*}(D)=\Gamma^{\perp}+(n-m) C_{0}^{\perp} \quad\left(\bmod \left\langle s_{i}^{\perp}, r_{i}^{\perp}, i=0, \ldots, 3\right\rangle\right),
$$

где через $\Gamma^{\perp}$ и $C_{0}^{\perp}$ обозначены $\tau^{\perp}$-инвариантные точные отображения $\Gamma$ и $C_{0}$ в $S^{\perp}$. В частности, редуцированньй образ $\Gamma^{\perp}$ в $S^{\sim}$ принадлежит $|\lambda(m, \nu)|$, где $\nu=\left(\nu_{i}\right)-$ тип $\Gamma^{\perp}$, т.е. $\nu_{i}:=\Gamma^{\perp} \cdot r_{i}^{\perp}$ при $i=0, \ldots, 3$, и

$$
D=\phi\left(\Gamma^{\perp}\right)+(n-m) C_{0}^{\sim}+\frac{1}{2}(n-m) \sum_{0 \leqslant i \leqslant 3} s_{i}^{\sim}+\frac{1}{2} \sum_{0 \leqslant i \leqslant 3}\left(\nu_{i}-\mu_{i}\right) r_{i}^{\sim} \in|\lambda(n, \mu)| .
$$

Таким образом, мы получили каноническое разложение $D$ в виде суммы неприводимого дивизора $\phi\left(\Gamma^{\perp}\right) \in|\lambda(m, \nu)|$ и другого эффективного слагаемого $\equiv \lambda(n, \mu)-$ $\lambda(m, \nu)$. Следовательно, $(m, \nu) \prec(n, \mu)$. Поэтому $D$ приводим, если и только если $(m, \nu) \neq(n, \mu)$. В последнем случае $D$ принадлежит естественному образу $|\lambda(m, \nu)|$ в $|\lambda(n, \mu)|$.

4.7. ПРЕДЛОЖЕНИЕ. Для любой пары $(n, \mu)$, где $\mu$ является n-подходящим вектором и $2 n+1 \geqslant \mu^{(2)}$, әлемент общего положения в $|\lambda(n, \mu)|$ - неприводимый дивизор.

ДокАЗАТЕЛЬСтво. Существует только конечное число таких пар $(m, \nu)$, что $(m, \nu) \prec(n, \mu)$ и $(m, \nu) \neq(n, \mu)$. Для каждой из них

$$
\operatorname{dim}|\lambda(m, \nu)|=\frac{1}{4}\left(2 m+1-\nu^{(2)}\right)<\frac{1}{4}\left(2 n+1-\mu^{(2)}\right)=\operatorname{dim}|\lambda(n, \mu)| .
$$

В частности, объединение соответствуюших собственно проективных подпространств, $\bigcup_{(m, \nu)}|\lambda(m, \nu)|$, которые, в соответствии с п. 4.6, параметризуют приводимые дивизоры в $|\lambda(n, \mu)|$, является замкнутьм собственным подмножеством. Следовательно, элемент общего положения в $|\lambda(n, \mu)|$ неприводим.

4.8. ПрЕДЛОЖЕнИЕ. Пусть $2 n+1>\mu^{(2)}$. Тогда полная линейная система $|\lambda(n, \mu)|$ обладает единственной базисной точкой. Эта базисная точка принадлежит $s_{0}^{\sim}$ и является гладкой точкой любого әлемента $x \in|\lambda(n, \mu)|$.

ДокАЗАТЕльСтво. Напомним сначала, что

$$
\lambda(n, \mu) \equiv \lambda(n-2, \mu)+2 C_{0}^{\sim}+\sum_{0 \leqslant i \leqslant 3} s_{i}^{\tilde{i}}
$$

и

$$
\lambda(n, \mu) \cdot C_{0}^{\tilde{N}}=\lambda(n, \mu) \cdot s_{j}^{\tilde{j}}=s_{0}^{\tilde{a}} \cdot s_{j}^{\tilde{j}}=0 \text { при } j=1,2,3,
$$

но $s_{0}^{\tilde{a}} \cdot C_{0}^{\sim}=1$. В частности,

$$
h^{\circ}\left(\mathscr{O}_{C_{0} \sim}\left(\lambda(n, \mu)-s_{0}^{\sim}\right)\right)=0 .
$$

Следовательно, соответствуюший дивизор $D^{\sim}=2 C_{0}^{\sim}+\sum_{1 \leqslant j \leqslant 3} s_{j}^{\sim}$; значит,

$$
h^{\circ}\left(\mathscr{O}_{D^{\sim}}\left(\lambda(n, \mu)-s_{0}^{\sim}\right)\right)=0 .
$$


Поэтому из последовательности когомологий, ассоциированной с точной последовательностью

$$
0 \rightarrow \mathscr{O}_{S} \sim(\lambda(n-2, \mu)) \rightarrow \mathscr{O}_{S} \sim\left(\lambda(n, \mu)-s_{0}^{\sim}\right) \rightarrow \mathscr{O}_{D} \sim\left(\lambda(n, \mu)-s_{0}^{\sim}\right) \rightarrow 0
$$

следует, что

$$
h^{\circ}\left(\mathscr{O}_{S} \sim\left(\lambda(n, \mu)-s_{0}^{\sim}\right)\right)=h^{\circ}\left(\mathscr{O}_{S} \sim(\lambda(n-2, \mu))\right)
$$

$\left(=h^{\circ}\left(\mathscr{O}_{S} \sim(\lambda(n, \mu))\right)-1\right.$ ввиду п. 3.4). С другой стороны, точна следующая последовательность:

$$
0 \rightarrow \mathscr{O}_{S} \sim\left(\lambda(n, \mu)-s_{0}^{\sim}\right) \rightarrow \mathscr{O}_{S} \sim(\lambda(n, \mu)) \rightarrow \mathscr{O}_{s_{0}}(\lambda(n, \mu)) \rightarrow 0
$$

Поскольку $H^{\circ}\left(\mathscr{O}_{S} \sim\left(\lambda(n, \mu)-s_{0}^{\sim}\right)\right)$ инъективно отображается как гиперплоскость $H^{\circ}\left(\mathscr{O}_{S} \sim(\lambda(n, \mu))\right)$, то образ $H^{\circ}\left(\mathscr{O}_{S} \sim(\lambda(n, \mu))\right)$ в $H^{\circ}\left(\mathscr{O}_{s_{0}}(\lambda(n, \mu))\right)$ является подпространством размерности 1 . При этом, поскольку $\lambda(n, \mu) \cdot s_{0}^{\sim}=1$, каждый приводимьй дивизор в $|\lambda(n, \mu)|$ пересекает $s_{0}^{\sim}$ только в одной и той же точке $s_{0}^{\sim}$. Последняя является гладкой точкой любого элемента $|\lambda(n, \mu)|$.

Докажем, наконец, индукцией по $n$, что $|\lambda(n, \mu)|$ не имеет другой базисной точки. Действительно, предположим, что $2 n+1-\mu^{(2)}=4$; в этом случае $\lambda(n, \mu)^{2}=\frac{1}{2}\left(2 n-1-\mu^{(2)}\right)=1$. Следовательно, любые два неприводимые элемента $|\lambda(n, \mu)|$ могут пересекаться только в одной точке, и эта точка является единственной базисной точкой, которая уже найдена. Предположим, далее, что $2 n+1-\mu^{(2)}>4$ (следовательно, $\left.2(n-2)+1-\mu^{(2)} \geqslant 4\right)$ и что $|\lambda(n-2, \mu)|$ имеет единственную базисную точку (в $\left.s_{0}^{\sim}\right)$. Известно, что для любого $x \in|\lambda(n-2, \mu)|$

$$
x+2 C_{0}^{\sim}+\sum_{0 \leqslant i \leqslant 3} s_{i}^{\sim} \in|\lambda(n, \mu)| .
$$

Очевидно, что любая базисная точка $|\lambda(n, \mu)|$ может лежать в $C_{0}^{\sim}$ или $s_{i}^{\sim}(i=0, \ldots, 3)$. Также известно, что

$$
\lambda(n, \mu) \cdot C_{0}^{\sim}=\lambda(n, \mu) \cdot s_{j}^{\sim}=0 \quad(j=1,2,3) .
$$

Следовательно, каждая базисная точка $|\lambda(n, \mu)|$ может лежать в $s_{0}^{\sim}$ и должна поэтому совпадать с ранее найденной.

4.9. СЛЕДСТВИЕ. Для любих $(n, \mu) \in \mathbb{N} \times \mathbb{N}^{4}$ maкux, ито $\mu-n$-nодходящий вектор, әлемент общего полоэсения в $|\lambda(n, \mu)|$ является гладкой неприводимой кривой рода $\frac{1}{4}\left(2 n+1-\mu^{(2)}\right)$.

ДоКАЗАТЕЛЬСТВО. Если $2 n+1<\mu^{(2)}$, то $|\lambda(n, \mu)|$ пусто и утверждение тривиально. Если же $2 n+1=\mu^{(2)}$, то единственный элемент $|\lambda(n, \mu)|$ является исключительной кривой (см. п. 4.1). Следовательно, эта кривая является гладкой, неприводимой кривой рода $0=\frac{1}{4}\left(2 n+1-\mu^{(2)}\right)$. Если $2 n+1>\mu^{(2)}$, то утверждение следует из предложения 4.8 и теоремы Бертини п. 3.3.3).

4.10. Изучение рациональных кривых $x \in|\lambda(n-2, \mu)|$ при любых парах $(n, \mu)$, удовлетворяюших неравенству $2 n+1>\mu^{(2)}$, представляется, вообше говоря, более трудным. Мы ограничимся в этом плане рассмотрением “первого неисключительного 
случая": $2 n+1-\mu^{(2)}=4$. В этом случае, $\operatorname{dim}|\lambda(n, \mu)|=1$, элемент общего положения в $|\lambda(n, \mu)|$ представляет собой эллиптическую кривую и существует единственная базисная точка (зависящая только от $n$ ), обозначаемая через $b(n) \in s_{0}$. Напомним также (п. 4.1), что $C_{\mu}^{\sim}$, единственньй элемент в $|\lambda(n-2, \mu)|$, является исключительной кривой и $y_{\infty}:=C_{\mu}^{\sim}+2 C_{0}^{\sim}+\sum_{0 \leqslant i \leqslant 3} s_{i}^{\sim}$ принадлежит $|\lambda(n, \mu)|$. Раздувая базисную точку $b(n)$, мы получаем поверхность $S_{n}^{\sim}$, снабженную моноидальным отображением $S_{n}^{\sim} \rightarrow S^{\sim}$ и естественной проекцией $S_{n}^{\sim} \rightarrow|\lambda(n, \mu)| \simeq \mathbb{P}^{1}(\mathbb{C})$. В $S_{n}^{\sim}$ точное отображение $C_{\mu}^{\sim}$ все еще является исключительной кривой. Стягивая ее, мы получаем в результате ращиональную эллиптическую поверхность $S_{(n, \mu)}^{\sim} \rightarrow|\lambda(n, \mu)| \simeq \mathbb{P}^{1}(\mathbb{C})$ с пикаровской группой $\operatorname{Pic}\left(S_{(n, \mu)}^{\sim}\right) \cong \mathbb{Z}^{10}$. Следовательно, характеристика Эйлера-Пуанкаре многообразия $S_{(n, \mu)}^{\sim}$ равна $\chi\left(S_{(n, \mu)}^{\sim}\right)=12$, и слой над $y_{\infty}$ равен $2 C_{0}^{\sim}+$ $\sum_{0 \leqslant i \leqslant 3} s_{i}^{\sim}$, где через $C_{0}^{\sim}$ и $s_{i}^{\sim}(i=0, \ldots, 3)$ обозначены их образы в $S_{(n, \mu)}^{\sim}$. Последние кривые имеют следуюшую диаграмму пересечений $\left(\right.$ в $\left.S_{(n, \mu)}^{\sim}\right)$ :

$$
C_{0}^{\sim} \cdot C_{0}^{\sim}=s_{i}^{\tilde{i}} \cdot s_{i}^{\tilde{T}}=-2, \quad C_{0}^{\sim} \cdot s_{i}^{\tilde{r}}=1, \quad s_{i}^{\sim} \cdot s_{j}^{\tilde{j}}=1 \quad(\text { если } i \neq j) .
$$

4.11. ЗАмечАния. 1) Для любого $y \in|\lambda(n, \mu)|$ обозначим через $\chi_{y}$ его характеристику Эйлера-Пуанкаре (ЕР-характеристику). Мы знаем, что обший слой пучка $S_{(n, \mu)}^{\sim} \rightarrow|\lambda(n, \mu)|$ является эллиптической кривой. Следовательно, функция $y \mapsto \chi_{y}$ обращается в нуль в конечном числе точек $|\lambda(n, \mu)|$, и так назьваемая формула Лефшеца (ср. [23; с. 509]) утверждает, что

$$
12=\chi\left(S_{(n, \mu)}^{\sim}\right)=\sum_{y \in|\lambda(n, \mu)|} \chi_{y} .
$$

2) ЕР-характеристика $\chi_{y} \neq 0$, если и только если многообразие $y$ приводимо или неприводимо, но сингулярно. Обозначим через Red (соответственно Irr) подмножество приводимых (соответственно неприводимых, но сингулярных) слоев. В частности, слой $y_{\infty}=2 C_{0}^{\sim}+\sum_{0 \leqslant i \leqslant 3} s_{i}^{\sim}$ принадлежит Red и все его неприводимые компоненты изоморфны $\mathbb{P}^{1}(\mathbb{C})$. Как следует из диаграммы пересечений $(13), \chi_{y_{\infty}}=6$. Остальные приводимые слои (Red $\left.\backslash\left\{y_{\infty}\right\}\right)$ находятся во взаимно однозначном соответствии с индексами $i=0, \ldots, 3$, для которых $\mu_{i}=0$ (п. 4.6). Зададим один из таких индексов $i_{0}$ и определим целочисленньй вектор $\left(\vec{e}_{i_{0}}(j)\right) \in \mathbb{N}^{4}$ по формуле $\vec{e}_{i_{0}}(j)=\delta_{i_{0} j}, j=0, \ldots, 3$. Поскольку $\mu_{i_{0}}=0$, то можно легко проверить, что $\left(n, \mu+2 \vec{e}_{i_{0}}\right) \prec(n, \mu)$. При этом исключительная кривая $C_{\mu+2}^{\sim} \vec{e}_{i_{0}}$ является единственным элементом в $\left|\lambda\left(n, \mu+2 \vec{e}_{i_{0}}\right)\right|$, приводимая кривая $y_{i_{0}}:=C_{\mu+2 \vec{e}_{i_{0}}}^{\sim}+r_{i_{0}}^{\sim}$ принадлежит $|\lambda(n, \mu)|$ и

$$
r_{i_{0}}^{\sim} \cdot \tilde{r_{i_{0}}}=-r_{i_{0}}^{\sim} \cdot C_{\mu+2 \vec{e}_{i_{0}}}^{\sim}=-2
$$

Следовательно, либо $r_{i_{0}}^{\sim}$ пересекает $C_{\mu+2 \vec{e}_{i_{0}}}^{\sim}$ в двух различных точках и $\chi_{y_{i_{0}}}=2$, либо $r_{i_{0}}^{\sim}$ касается $C_{\mu+2 \vec{e}_{i_{0}}}^{\sim}$ и $\chi_{y_{i_{0}}}=3$.

3) C другой стороны, Irr определяется как подмножество всех сингулярных неприводимых дивизоров в $|\lambda(n, \mu)|$. Арифметический род любого $y \in|\lambda(n, \mu)|$ равен $\frac{1}{4}\left(2 n+1-\mu^{(2)}\right)=1$, и мы заключаем, что Irr состоит из всех неприводимых рациональных кривых в $|\lambda(n, \mu)|$. Каждая такая кривая $y \in \operatorname{Irr}$ должна иметь единственную сингулярную точку - либо узел, в этом случае $\chi_{y}=1$, либо клюв, 
тогда $\chi_{y}=2$. Обозначим, как обычно, через $R(n, \mu, X)$ множество классов эквивалентности минимально-гиперэллиптических касательных накрытий степени $n$ и типа $\mu$ над $(X, q)$. Принимая во внимание уравнение (14) и сделанные вьше замечания относительно $\chi_{y}$ при $y \in \operatorname{Red} \cup \operatorname{Irr}$, мы немедленно получаем следуюшие ограничения для \# $R(n, \mu, X)$, размерности $R(n, \mu, X)$.

4.12. ТЕОРемА. Для любой nары $(n, \mu) \in \mathbb{N} \times \mathbb{N}^{4}$ такой, что $\mu$ является $n$-подходящим и $\mu^{(2)}=2 n-3, \# R(n, \mu, X)$ ограничено следующим образом:

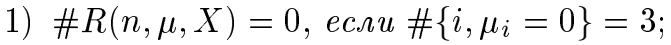

2) $0 \leqslant \# R(n, \mu, X) \leqslant 2$, если \#\{i, $\left.\mu_{i}=0\right\}=2$;

3) $2 \leqslant \# R(n, \mu, X) \leqslant 4$, если \#\{i, $\left.\mu_{i}=0\right\}=1$;

4) $3 \leqslant \# R(n, \mu, X) \leqslant 6$, если \#\{i, $\left.\mu_{i}=0\right\}=0$.

4.13. ЗАмечАниЕ. Неравенства п. 4.12.2) могут быть улучшены. Действительно, в доказательстве п. 9.4 будет приведена конструкция двух различных (непримитивных) элементов $R(n, \mu, X)$, в то время как $\#\left\{i, \mu_{i}=0\right\}=2$. Следовательно, $\# R(n, \mu, X)=2\left(\right.$ если \# $\left.\left\{i, \mu_{i}=0\right\}=2\right)$.

\section{5. Канонические $\theta$-характеристики и их потенциалы:$$
\text { случай } \mu^{(2)}=2 n+1[41]
$$

5.1. Пусть $\pi:(\Gamma, p) \rightarrow(X, q)$ - некоторое гиперэллиптическое касательное накрытие степени $n \geqslant 1$. Напомним, что для любого $\xi \in W(\Gamma)$ прообраз канонического тэта-дивизора $\Theta_{\Gamma}$ по действию орбитного морфизма

$$
\operatorname{Orb}_{\xi}: X \rightarrow W(\Gamma), \quad q^{\prime} \mapsto \xi\left(\pi^{*}\left(q^{\prime}-q\right)\right)=\xi \otimes \mathscr{O}_{\Gamma}\left(\pi^{*}\left(q^{\prime}-q\right)\right),
$$

является дивизором степени $n$ в $X$,

$$
\operatorname{Orb}_{\xi}^{*}\left(\Theta_{\Gamma}\right)=\sum_{i=1}^{n} \alpha_{i}
$$

При этом $\sum_{i=1}^{n} \alpha_{i}$ принадлежит $\mathrm{KdV}(n, X)$, а именно, $n$-му $\mathrm{K} д В$-локусу, и единственным образом определяется классом изоморфизмов данных $(\pi ; \xi)$ (см. [1] или $[38 ; \S \S 2.7$, $2.9]$ ). Также известно, что эллиптический конечно-зонньй потенщиал, ассоциированньй с $(\pi ; \xi)$, равен

$$
u_{\xi}(x)=2 \sum_{i=1}^{n} \wp\left(x-\alpha_{i}\right)
$$

(п. 1.9 или $[39 ; \S 7.10])$. Более того, для любого $\alpha=q^{\prime}-q \in \mathrm{Jac} X(\simeq X)$ эллиптический конечно-зонный потенциал, ассоциированньй с $\xi\left(\pi^{*}\left(q^{\prime}-q\right)\right)$, равен

$$
2 \sum_{i=1}^{n} \wp\left(x-\left(\alpha_{i}-\alpha\right)\right)
$$

Другими словами,

$$
u_{\xi\left(\pi^{*}\left(q^{\prime}-q\right)\right)}(x)=u_{\xi}(x+\alpha) .
$$


Нахождение $\operatorname{Orb}_{\xi}^{*}\left(\Theta_{\Gamma}\right)$ позволяет вычислить кратность пересечения $\Theta_{\Gamma}$ с $\operatorname{Orb}_{\xi}(X)$ в любой точке $\xi^{\prime}=\operatorname{Orb}_{\xi}\left(q^{\prime}\right), q^{\prime} \in X$. Нашим основным инструментом (п. 5.4) является непосредственное следствие такой ограниченной версии формулы Фэя-Сигала-Вильсона.

5.2. ПреДЛОЖЕНИЕ. Пусть $\pi:(\Gamma, p) \rightarrow(X, q)$ - гиперәллиптическое касательное накрытие и $\xi$ - произвольная точка $W(\Gamma)$. Тогда кратность пересечения $\Theta_{\Gamma} и \operatorname{Orb}_{\xi}(X)$ в $\xi$ равна

$$
\operatorname{mult}_{\xi}\left(\Theta_{\Gamma}, \operatorname{Orb}_{\xi}(X)\right)= \begin{cases}\left(2 h^{\circ}(\xi)-1\right) \cdot h^{\circ}(\xi), & \text { если } h^{\circ}(\xi)>h^{\circ}(\xi(-p)), \\ \left(2 h^{\circ}(\xi)+1\right) \cdot h^{\circ}(\xi), & \text { если } h^{\circ}(\xi)=h^{\circ}(\xi(-p)) .\end{cases}
$$

5.3. ЗАмЕчАнИя. 1) Из формулы (15) следует, что кратность пересечения является треугольньм числом, которое возрастает с увеличением $h^{\circ}(\xi)$. В частности, если $h^{\circ}(\xi(-m p))>0$ для некоторого $m \geqslant 0$, то мы получаем следуюшую нижнюю границу:

$$
\operatorname{mult}_{\xi}\left(\Theta_{\Gamma}, \operatorname{Orb}_{\xi}(X)\right) \geqslant \frac{1}{2}(m+1)(m+2) .
$$

2) Для любого $i=0, \ldots, 3 \xi_{i}:=\xi\left(\pi^{*}\left(\omega_{i}-\omega_{0}\right)\right)$ обладает той же $X$-орбитой, что и $\xi$, соответствуюший потенциал равен $u_{\xi_{i}}(x) \equiv u_{\xi}\left(x-\omega_{i}\right)$ и

$$
\sum_{i=0}^{3} \operatorname{mult}_{\xi_{i}}\left(\Theta_{\Gamma}, \operatorname{Orb}_{\xi}(X)\right) \leqslant \Theta_{\Gamma} \cdot \operatorname{Orb}_{\xi}(X)=\operatorname{deg}(\pi)=n .
$$

3) Дивизоры $\left\{2 n p, 2 \pi^{*}\left(\omega_{i}\right), i=0, \ldots, 3\right\}$ имеют одну и ту же степень и являются неподвижньми точками при действии гиперэллиптической инволюции кривой $\Gamma$. Следовательно, они линейно эквивалентны. Обозначив через $g$ арифметический род $\Gamma$ и зная, что канонический пучок равен $\omega_{\Gamma} \simeq \mathscr{O}_{\Gamma}((2 g-2) p)$, мы заключаем, что обратимые пучки:

$$
\begin{aligned}
& \xi_{A, i}=\mathscr{O}_{\Gamma}\left((g-1) p+\pi^{*}\left(\omega_{i}-q\right)\right), \quad i=0, \ldots, 3, \\
& \xi_{B, i}=\mathscr{O}_{\Gamma}\left((g-1-n) p+\pi^{*}\left(\omega_{i}\right)\right),
\end{aligned}
$$

являются $\theta$-характеристиками на $Г$. Если $\pi$ - исключительное накрытие, мы можем найти соответствуюший эллиптический конечно-зонньй потенциал, как это будет показано далее (пा. 5.4, 5.5). Первым основным результатом в этом направлении является нахождение общей нижней границы для соответствуюших кратностей пересечения, полученной в результате непосредственного применения пп. 5.2 и 5.3.1) (этот результат справедлив только для минимально-гиперэллиптических касательных накрытий). Благодаря тому, что при $j=1,2,3 \xi_{A, j}$ и $\xi_{B, j}$ принадлежат $\operatorname{Orb}_{\xi_{A, 0}}(X)$ и $\operatorname{Orb}_{\xi_{B, 0}}(X)$ соответственно, удается вычислить $u_{\xi_{A, 0}}$ и $u_{\xi_{B, 0}}$ (см. п. 5.3.2)).

5.4. Теорема. Пусть $\pi:(\Gamma, p) \rightarrow(X, q)$ - некоторое минимально-гиперэллиптическое касательное накрытие степени $n$, типа $\mu$ и арифметического рода 
$g:=\frac{1}{2}\left(\mu^{(1)}-1\right)$, и пусть $\xi_{A, i}$ и $\xi_{B, i}-$ канонические $\theta$-характеристики, определенные в $n$. 5.3.3) для любых полупериодов $\omega_{i} \in(X, q)_{2}$. Пусть также $m_{i}^{A, 0}$ (соответственно $m_{i}^{B, 0}$ ) обозначает кратность пересечения

$$
\begin{aligned}
m_{i}^{A, 0} & :=\operatorname{mult}_{\xi_{A, i}}\left(\Theta_{\Gamma}, \operatorname{Orb}_{\xi_{A, 0}}(X)\right) \\
\left(\text { coответственно } m_{i}^{B, 0}\right. & \left.:=\operatorname{mult}_{\xi_{B, i}}\left(\Theta_{\Gamma}, \operatorname{Orb}_{\xi_{B, 0}}(X)\right)\right) .
\end{aligned}
$$

Тогда $m_{0}^{A, 0}=\frac{1}{2} g(g+1)$ и при $j=1,2,3$ и $i=0, \ldots, 3$ имеют место следующие нижние границы:

$$
m_{j}^{A, 0} \geqslant \frac{1}{2}\left(g-\mu_{0}-\mu_{j}\right)\left(g-\mu_{0}-\mu_{j}+1\right), \quad m_{i}^{B, 0} \geqslant \frac{1}{2}\left(g-\mu_{i}\right)\left(g-\mu_{i}+1\right) .
$$

5.5. СлЕДСТВИЕ. Пусть $\pi_{\mu}:(\Gamma, p) \rightarrow(X, q)$ - исключительное накрытие типа $\mu\left(\right.$ степени $n:=\frac{1}{2}\left(\mu^{(2)}-1\right)$ и арифметического рода $\left.g:=\frac{1}{2}\left(\mu^{(1)}-1\right)\right)$. Тогда четные әллиптические конечно-зонные потенциаль, ассочиированные с $\theta$-характеристиками $\xi_{A, 0}=\mathscr{O}_{\Gamma}((g-1) p)$ и $\xi_{B, 0}=\mathscr{O}_{\Gamma}\left((g-1-n) p+\pi^{*}(q)\right)$, coответственно равны:

$$
\begin{aligned}
& u_{\xi_{A, 0}}(x)=g(g+1) \wp(x)+\sum_{1 \leqslant j \leqslant 3}\left(g-\mu_{0}-\mu_{j}\right)\left(g-\mu_{0}-\mu_{j}+1\right) \wp\left(x-\omega_{j}\right), \\
& u_{\xi_{B, 0}}(x)=\sum_{0 \leqslant i \leqslant 3}\left(g-\mu_{i}\right)\left(g-\mu_{i}+1\right) \wp\left(x-\omega_{i}\right) .
\end{aligned}
$$

ДОКАЗАТЕЛЬСТВО. Легко проверить, что

$$
\begin{gathered}
\sum_{0 \leqslant i \leqslant 3}\left(g-\mu_{i}\right)\left(g-\mu_{i}+1\right)=\mu^{(2)}-1, \\
g(g+1)+\sum_{1 \leqslant j \leqslant 3}\left(g-\mu_{0}-\mu_{j}\right)\left(g-\mu_{0}-\mu_{j}+1\right)=\mu^{(2)}-1 .
\end{gathered}
$$

Применяя формулы нижних границ (20) и п. 5.3.2), мы немедленно заключаем, что

$$
\begin{aligned}
& \mu^{(2)}-1 \leqslant 2 \sum_{0 \leqslant i \leqslant 3} m_{i}^{A, 0} \leqslant 2 n, \\
& \mu^{(2)}-1 \leqslant 2 \sum_{0 \leqslant i \leqslant 3} m_{i}^{B, 0} \leqslant 2 n .
\end{aligned}
$$

Далее, в нашем (исключительном) случае $\mu^{(2)}-1$ равно $2 n$. Следовательно, при $j=$ $1,2,3$ и $i=0, \ldots, 3$ получаем

$$
\begin{aligned}
& 2 m_{j}^{A, 0}=\left(g-\mu_{0}-\mu_{j}\right)\left(g-\mu_{0}-\mu_{j}+1\right), \\
& 2 m_{i}^{B, 0}=\left(g-\mu_{i}\right)\left(g-\mu_{i}+1\right),
\end{aligned}
$$

и $u_{\xi_{A, 0}}(x)$, так же как и $u_{\xi_{B, 0}}(x)$, даны в $(21)$. 
5.6. ЗАмЕчАниЕ. В соответствии со следствием 5.5 , если $\pi$ - исключительное накрытие, то эллиптические конечно-зонные потенциалы, ассоциированные с множеством $\theta$-характеристик $\left\{\xi_{A, i}, \xi_{B, i}, i=0, \ldots, 3\right\}$, могут иметь полюса только в полупериодах кривой $(X, q)$. Далее будет показано, что это свойство является определяющим для канонических $\theta$-характеристик исключительных накрытий.

5.7. ОПРЕДЕлЕНИЕ. Пусть $\pi:(\Gamma, p) \rightarrow(X, q)$ - гиперэллиптическое касательное накрытие, и пусть $g$ обозначает арифметический род кривой Г. Будем говорить, что функция $u(x)$ является порождающим потенииалом, ассоциированнылм $с \pi$, если $u(x)$ - эллиптический конечно-зонный потенщиал, ассоциированньй с данньми $\left(\pi ; \mathscr{O}_{\Gamma}((g-1) p)\right)$.

5.8. Теорема ([40], [41], см. также [18]). Для любы $\left(a_{i}\right) \in \mathbb{N}^{4} \backslash\{\overrightarrow{0}\}$ cyществует исключительное накрытие $\pi:(\Gamma, p) \rightarrow(X, q)$ такое, что четная функиия

$$
u(x)=\sum_{0 \leqslant i \leqslant 3} a_{i}\left(a_{i}+1\right) \wp\left(x-\omega_{i}\right)
$$

является әллиптическим конечно-зонным потенциалом, ассоциированнылм с одной из восьми канонических $\theta$-характеристик, $\left\{\xi_{A, i}, \xi_{B, i}, i=0, \ldots, 3\right\}$, на Г. Степень $\pi$ равна

$$
n:=\frac{1}{2} \sum_{0 \leqslant i \leqslant 3} a_{i}\left(a_{i}+1\right),
$$

а арифметический род Г равен

$$
g:=\frac{1}{2} \max \left(2 M, 1+\Sigma-\left(1+(-1)^{\Sigma}\right)\left(m+\frac{1}{2}\right)\right),
$$

$2 \partial e$

$$
\Sigma=\sum_{0 \leqslant i \leqslant 3} a_{i}, \quad M=\max \left\{a_{i}\right\} \quad u \quad m=\min \left\{a_{i}\right\} .
$$

При этом такая функиия (5) является порождающим потенциалом, если и только если

$$
a_{0}=M \quad u \quad 2 M \geqslant \Sigma-\left(1+(-1)^{\Sigma}\right) m
$$

Эскиз ДоКАЗАТЕЛЬствА. Доказательство, данное в $[41 ; \oint \S 4.4,5.3]$, довольно элементарно и производится следующим образом. Для любых $\left(a_{i}\right) \in \mathbb{N}^{4} \backslash\{\overrightarrow{0}\}$ существует специальньй вектор $\mu \in \mathbb{N}^{4}$, задаваемый явной формулой, выведенной в [ 41 ; $\S \S 4.4,5.4 .3]$, и такой, что $\mu-n$-подходящий с $n:=\frac{1}{2} \sum_{0 \leqslant i \leqslant 3} a_{i}\left(a_{i}+1\right)$ и $\mu^{(2)}=2 n+1$. Пусть $\pi:(\Gamma, p) \rightarrow(X, q)$ - исключительное накрытие типа $\mu$ и $\left\{\xi_{A, i}, \xi_{B, i}, i=0, \ldots, 3\right\}$ - множество $\theta$-характеристик, определенное ранее в п. 5.3.3). Непосредственная проверка формулы (21), проведенная в п. 5.5, показьвает, что

$$
u(x)=\sum_{0 \leqslant i \leqslant 3} a_{i}\left(a_{i}+1\right) \wp\left(x-\omega_{i}\right)
$$

- эллиптический конечно-зонньй потенциал, ассоциированньй с $\xi_{A, i}$ или $\xi_{B, i}$ при некотором $i=0, \ldots, 3$. Наконец, арифметический род $\Gamma$, как известно, равен $g:=$ $\frac{1}{2}\left(\mu^{(1)}-1\right)$. Выражая $\left(\mu_{i}\right)$ через $\left(a_{i}\right)$ по формуле из $[41 ; \S \S 4.4,5.4 .3]$, мы немедленно получаем выражение (25). 


\section{6. Вывод новых эллиптических конечно-зонных потенциалов}

6.1. Мы применили в разделе 5 формулу кратности Фея-Сигала-Вильсона (п. 5.2) и формулу для нижних гранищ (20) к произвольному исключительному накрытию, получив в результате полное описание соответствуюших канонических $\theta$-характеристик (п. 5.3.2)) в терминах ассоциированных эллиптических конечно-зонных потенциалов (пш. 5.5, 5.8). На основе результатов п. 5.2, п. 5.4 (формула (20)) и пп. 5.5, 5.8 мы исследуем далее ("первый неисключительный") случай $\mu^{(2)}=2 n-3$ и находим бесконечное семейство четных эллиптических конечно-зонных потенциалов вида (6), где $\left(a_{i}\right) \in \mathbb{N}^{4}$ и $\rho$ удовлетворяет уравнению (7) (см. п. 6.3).

6.2. ПРЕДЛОЖЕНИЕ. Пусть $\left(a_{i}\right) \in \mathbb{N}^{4} u \rho \in X \backslash\left\{\omega_{i}, i=0, \ldots, 3\right\}$ таковьи, что четная функиия (6) является эллиптическим конечно-зонным потенциалом. Тогда р удовлетворяет “фундаментальному” уравнению (7).

ДокАЗАТЕльство. Обозначим через $v(x, t)$ единственное решение уравнения $\mathrm{K}$ дВ, имеющее в качестве "начального значения" $v(x, 0) \equiv u(x)$. Нами уже показано, что существует множество из $n:=2+\frac{1}{2} \sum_{i=0}^{3} a_{i}\left(a_{i}+1\right)$ различных аналитических функций $\left\{x_{k}(t), 1 \leqslant k \leqslant n\right\}$ таких, что $v(x, t)=2 \sum_{k=1}^{n} \wp\left(x-x_{k}(t)\right)$, и дивизор $\sum_{k=1}^{n}\left(x_{k}(t)\right)$ принадлежит $\operatorname{KdV}(n, X)$ при любом $t \in \mathbb{C}$ (см., например, п. 1.9). Следовательно, $\sum_{k=1}^{n}\left(x_{k}(t)\right)$ стремится при $t \rightarrow 0$ к дивизору

$$
(\rho)+(-\rho)+\frac{1}{2} \sum_{i=0}^{3} a_{i}\left(a_{i}+1\right)\left(\omega_{i}\right)
$$

удовлетворяя (в частности) уравнениям

$$
\sum_{k=2}^{n} \wp^{\prime}\left(x_{2}(t)-x_{k}(t)\right)=0 \quad(t \neq 0)
$$

Переходя к пределу $(t \rightarrow 0)$ в последнем равенстве, мы получим

$$
\sum_{k=2}^{n} \wp^{\prime}\left(x_{1}(0)-x_{k}(0)\right)=\wp^{\prime}(2 \rho)+\frac{1}{2} \sum_{i=0}^{3} a_{i}\left(a_{i}+1\right) \wp^{\prime}\left(\rho-\omega_{i}\right)=0
$$

Поскольку из классических формул следует, что $8 \wp^{\prime}(2 \rho)=\sum_{i=0}^{3} \wp^{\prime}\left(\rho-\omega_{i}\right)$, мы в результате получаем (7), как и предполагалось.

6.3. ТЕОРемА. Пусть $[\pi:(\Gamma, p) \rightarrow(X, q)] \in R(n, \mu, X)$ - минимально-гиперәллиптическое касательное накрытие типа $\mu$ (и, следовательно, арифметического рода $\left.g:=\frac{1}{2}\left(\mu^{(1)}-1\right)\right)$ и $n-$ такая степень, что $\mu^{(2)}=2 n-3$. Тогда для любой канонической $\theta$-характеристики $\xi \in\left\{\xi_{A, k}, \xi_{B, k}, k=0, \ldots, 3\right\}$ существуют иелочисленный вектор $\vec{a} \in \mathbb{N}^{4}$ и точка $\rho \in X(2 \rho \neq 0)$, удовлетворяющие уравнению (7), такие, что әллиптический конечно-зонный потенциал, ассочиированный $с(\pi ; \xi)$, определяется по формуле $(6)$. 
Пусть $\xi=\xi_{A, k}\left(\xi=\xi_{B, k}\right)$ и обозначим вектор $\vec{a}=\left(a_{i}\right) \in \mathbb{N}^{4}$, появляющийся в (6), как $A^{k}(\mu)$ (соответственно как $\left.B^{k}(\mu)\right)$. Тогда $\vec{a}=A^{\circ}(\mu)\left(\vec{a}=B^{\circ}(\mu)\right)$ определяется по следующей формуле:

$$
\begin{aligned}
a_{0}= & g=\frac{1}{2}\left(\mu^{(1)}-1\right) \quad \text { u } \quad a_{j}=\frac{1}{2}\left(\left|\mu^{(1)}-2 \mu_{0}-2 \mu_{j}\right|-1\right) \quad \text { nри } \quad j=1,2,3 \\
& \left(\text { соответственно } a_{i}=\frac{1}{2}\left(\left|\mu^{(1)}-2 \mu_{i}\right|-1\right) \quad \text { при } i=0, \ldots, 3\right) .
\end{aligned}
$$

Зная, с другой стороньи, что $u_{\xi_{A, k}}(x) \equiv u_{\xi_{A, 0}}\left(x-\omega_{k}\right)$ u что $u_{\xi_{B, k}}(x) \equiv$ $u_{\xi_{B, 0}}\left(x-\omega_{k}\right)$, мы можем вывести из (26) явные формуль для $A^{k}(\mu)$ и $B^{k}(\mu)$ $(k=1,2,3)$.

ДокАЗАТЕЛЬСТво. Предположим, что $\xi$ равно либо $u_{\xi_{A, 0}}$, либо $u_{\xi_{B, 0}}$, и обозначим через $m_{i}$ кратность пересечения в точке $\xi_{i}:=\xi\left(\pi^{*}\left(\omega_{i}-\omega_{0}\right)\right)(i=0, \ldots, 3)$ между $\Theta_{\Gamma}$ и $\operatorname{Orb}_{\xi}(X)$. Выберем $\vec{a}$ равньм $A^{\circ}(\mu)$ (соответственно $B^{\circ}(\mu)$ ). Непосредственное вычисление показьвает, что в обоих случаях мы имеем

$$
\mu^{(2)}-1=\sum_{i=0}^{3} a_{i}\left(a_{i}+1\right)
$$

С другой стороны, из п. 5.3.2) и формулы для нижней гранищы (20) следует, что

$$
\sum_{i=0}^{3} m_{i} \leqslant n \quad \text { и } \frac{1}{2} a_{i}\left(a_{i}+1\right) \leqslant m_{i}, \quad i=0, \ldots, 3 .
$$

Следовательно,

$$
\mu^{(2)}-1=\sum a_{i}\left(a_{i}+1\right) \leqslant \sum_{i=0}^{n} 2 m_{i} \leqslant 2 n .
$$

Далее, в рассматриваемом нами случае $\mu^{(2)}-1=2 n-4$, что приводит к неравенствам

$$
n-2=\frac{1}{2} \sum_{i=0}^{3} a_{i}\left(a_{i}+1\right) \leqslant \sum_{i=0}^{3} m_{i} \leqslant n .
$$

Мы можем также утверждать, что эллиптический конечно-зонньй потенциал $u_{\xi}(x)-$ четная функция, имеющая (по крайней мере) два полюса вне множества полупериодов $\left\{\omega_{i}\right\}$. В противном случае $u_{\xi}(x)$ должен был бы быть одним из потенциалов п. 5.8 и $\pi$ - исключительным накрытием (противоречие!). В результате мы заключаем, что

$$
\sum_{i=0}^{3} m_{i}=n-2=\frac{1}{2} \sum_{i=0}^{3} a_{i}\left(a_{i}+1\right)
$$

в то время как $m_{i} \geqslant \frac{1}{2} a_{i}\left(a_{i}+1\right)$ при $i=0, \ldots, 3$. Следовательно, $m_{i}=\frac{1}{2} a_{i}\left(a_{i}+1\right)$ при $i=0, \ldots, 3$, как было заявлено, и существует $\rho \in X \backslash\left\{\omega_{i}\right\}$, удовлетворяющее фундаментальному уравнению $(7)$, такое, что $u_{\xi}(x)$ определяется по формуле (6) (п. 6.2). 
6.4. ЗАмечАния. 1) Каждому $\pi \in R(n, \mu, X)$, определенному, как и ранее (при $\left.n=\frac{1}{2}\left(\mu^{(2)}+3\right)\right)$, мы сопоставили восемь эллиптических конечно-зонных потенциалов в (6). Соответствующие целочисленные векторы $\vec{a} \in \mathbb{N}^{4}$ удовлетворяют равенству

$$
\sum_{i=0}^{3} a_{i}\left(a_{i}+1\right)=2 n-4=\mu^{(2)}-1
$$

и зависят только от $\mu$ и выбора $\theta$-характеристики. Восемь отображений $A^{k}$ и $B^{k}$, $\mu \mapsto \vec{a}(k=0, \ldots, 3)$, определяемые каждым выбором, тщательно изучены в $[20 ; \S 4$ и $\S 5]$ : они инъективны и в совокупности заполняют все $\mathbb{N}^{4}$.

2) Обозначим далее через $\mathbb{M} \subset \mathbb{N}^{4}$ подмножество всех возможных типов,

$$
\mathbb{M}=\left\{\mu=\left(\mu_{i}\right) \in \mathbb{N}^{4} \mid \mu_{0}+1 \equiv \mu_{1} \equiv \mu_{2} \equiv \mu_{3}(\bmod 2)\right\}
$$

и через $M^{\# k}(k=0, \ldots, 3)$ подмножество $\mathbb{M}$, образованное всеми типами, имеюшими $k$ нулевых координат:

$$
\mathbb{M}^{\# k}=\left\{\mu \in \mathbb{M} \mid \#\left\{j, \mu_{j}=0\right\}=k\right\}
$$

Напомним, что для любой эллиптической кривой $(X, q)$ и любого типа $\mu \in \mathbb{M}$ множество $R(n, \mu, X)\left(n=\frac{1}{2}\left(\mu^{(2)}+3\right)\right)$ пусто тогда и только тогда, когда $\mu$ принадлежит $\mathbb{M}^{\# 3}=\{(2 k+1,0,0,0), k \in \mathbb{N}\}$. C другой стороны, непосредственная проверка показывает, что $\mu \in \mathbb{M} \# 3$ тогда и только тогда, когда для всех $k=0, \ldots, 3 A^{k}(\mu)$ и $B^{k}(\mu)$ принадлежат $\{(a, a, a, a), a \in \mathbb{N}\}$. Нетрудно вьвести следуюшее обратное 6.3 утверждение.

6.5. СЛЕДСТВИЕ. Для любого вектора $\vec{a}$, принадлежащего дополнению $\kappa\{(b, b, b, b), b \in \mathbb{N}\} \quad$ (т.е. $\left.\vec{a} \in \mathbb{N}^{4} \backslash(1,1,1,1) \mathbb{N}\right)$, существует иисло $\rho \in X \backslash$ $\left\{\omega_{i}\right\}$, удовлетворяющее соотношению (7), такое, что (6) - один из эллиптических конечно-зонных “канонически" ассочиированных потенциалов (n. 6.3) с некоторым $\pi \in R(n, \mu, X)$, где $\mu$ такое, что $\vec{a} \in\left\{A^{k}(\mu), B^{k}(\mu)\right\}, u$

$$
n:=\frac{1}{2}\left(\mu^{(2)}+3\right)=2+\frac{1}{2} \sum_{i=0}^{3} a_{i}\left(a_{i}+1\right) .
$$

ДоКАЗАТЕЛЬСТВо. Для любого такого $\vec{a}$ существуют $\mu \in \mathbb{M} \backslash \mathbb{M} \# 3$ и $k \in\{0, \ldots, 3\}$ такие, что либо $A^{k}(\mu)=\vec{a}$, либо $B^{k}(\mu)=\vec{a}$. При этом множество $R(n, \mu, X)(n=$ $\left.\frac{1}{2}\left(\mu^{(2)}+3\right)\right)$ непусто. Следовательно, эллиптический конечно-зонньй потенциал, ассоциированньй в п. 6.3 либо с $\left(\pi ; \xi_{A, k}\right)$, либо с $\left(\pi ; \xi_{B, k}\right)$ при любом $\pi \in R(n, \mu, X)$, определяется по формуле (6) для некоторого $\rho$ из $X$, удовлетворяющего (7) (п. 6.3).

6.6. ЗАмечАниЕ. (Случай $\vec{a} \in\{(b, b, b, b), b \in \mathbb{N}\}$.) Предположим обратное, т.е. что $\vec{a}=(b, b, b, b)$ для некоторого $b \in \mathbb{N}$, и выберем $\rho \in X$, удовлетворяющее

$$
\sum_{i=0}^{3}\left(2 a_{i}+1\right)^{2} \wp^{\prime}\left(\rho-\omega_{i}\right)=(2 b+1)^{2} \sum_{i=0}^{3} \wp^{\prime}\left(\rho-\omega_{i}\right)=0 .
$$


Тогда $2 \rho$ - ненулевой полупериод (поскольку $\left.\sum_{i=0}^{3} \wp^{\prime}\left(x-\omega_{i}\right) \equiv 8 \wp^{\prime}(2 x)\right)$ и $u(x)=$ $2 \wp(x-\rho)+2 \wp(x+\rho)+b(b+1) \sum_{i=0}^{3} \wp\left(x-\omega_{i}\right)$ - непримитивньй $($ п. $\left.1.13,2)\right)$ эллиптический конечно-зонный потенщиал, ассоциированньй с исключительным накрытием типа $\mu=(2 b+1,2,0,0),(2 b+1,0,2,0)$ или $(2 b+1,0,0,2)$. Подробнее, предположим, что $2 \rho=\omega_{1}$, и обозначим через $\varphi: X \rightarrow X /\left\langle\omega_{1}\right\rangle=: Y$ каноническую проекцию и через $\widehat{\varphi}: Y \rightarrow X-$ дуальный гомеоморфизм. В частности, $\operatorname{Ker}(\varphi)=\left\{\omega_{0}, \omega_{1}\right\}$ и композиция $\varphi$ с $\widehat{\varphi}$ дает “умножение на 2 ". Следовательно,

$$
\omega_{0}^{\prime}:=\varphi\left(\omega_{0}\right)=\varphi\left(\omega_{1}\right), \quad \omega_{1}^{\prime}:=\varphi\left(\omega_{2}\right)=\varphi\left(\omega_{3}\right), \quad \omega_{2}^{\prime}:=\varphi(\rho), \quad \omega_{3}^{\prime}:=\varphi\left(\rho+\omega_{2}\right)
$$

- четыре полупериода $Y$. Аналогично мы можем легко проверить, что

$$
\widehat{\varphi}\left(\omega_{0}^{\prime}\right)=\widehat{\varphi}\left(\omega_{1}^{\prime}\right)=\omega_{0} \text { и } \widehat{\varphi}\left(\omega_{2}^{\prime}\right)=\widehat{\varphi}\left(\omega_{3}^{\prime}\right)=\omega_{1} .
$$

Рассмотрим исключительное накрытие $\pi^{\prime}:(\Gamma, p) \rightarrow\left(Y, q^{\prime}\right)$ степени $m:=b^{2}+b+1$, арифметического рода $g:=b+1$ и типа $\nu$, равного либо $\nu=(b+1, b, 1,1)-$ если $b \equiv 1$ $(\bmod 2)$, либо $\nu=(b, b+1,1,1)-$ если $b \equiv 0(\bmod 2)$. В этом случае эллиптический конечно-зонньй потенциал, ассоциированньй с алгебраическими данными

$$
\left(\pi^{\prime} ; \xi\right):=\left(\pi^{\prime} ; \mathscr{O}_{\Gamma}\left((g-1-m) p+\left(\pi^{\prime}\right)^{*}\left(\omega_{2}^{\prime}\right)\right)\right)
$$

равен (см. п. 5.5):

$$
v(y)=b(b+1) \wp_{Y}(y)+b(b+1) \wp_{Y}\left(y-\omega_{1}^{\prime}\right)+2 \wp_{Y}\left(y-\omega_{2}^{\prime}\right),
$$

где мы обозначили через $\wp_{Y}$ стандартную ю-функцию Вейерштрасса кривой $\left(Y, q^{\prime}\right)$. Преобразуя потенщиал $v(y)$ с помощью $\varphi: X \rightarrow Y$, мы получаем (с точностью до постоянной) эллиптический конечно-зонньй потенциал, ассоциированньй с $(\pi ; \xi):=$ $\left(\widehat{\varphi} \circ \pi^{\prime} ; \xi\right)\left(\right.$ см. п. 1.14). Теперь мы также знаем, что $\wp_{Y}(\varphi(x)) \equiv \wp(x)+\wp\left(x-\omega_{1}\right)+$ const. Следовательно,

$$
\begin{aligned}
& \wp_{Y}\left(\varphi(x)-\omega_{1}^{\prime}\right)=\wp_{Y}\left(\varphi\left(x-\omega_{2}\right)\right) \equiv \wp\left(x-\omega_{2}\right)+\wp\left(x-\omega_{3}\right)+\text { const } \\
& \wp_{Y}\left(\varphi(x)-\omega_{2}^{\prime}\right)=\wp_{Y}(\varphi(x-\rho)) \equiv \wp(x-\rho)+\wp(x-\rho)+\text { const }
\end{aligned}
$$

(поскольку $\left.-\rho-\omega_{1}=\rho\right)$. В результате мы заключаем, что, с точностью до постоянной,

$$
u(x)=2 \wp(x-\rho)+2 \wp(x+\rho)+b(b+1) \sum_{i=0}^{3} \wp\left(x-\omega_{i}\right)=v(\varphi(x))
$$

является непримитивным эллиптическим конечно-зонным потенциалом, ассоциированным с алгебраическими данными

$$
\left(\widehat{\varphi} \circ \pi^{\prime} ; \mathscr{O}_{\Gamma}\left((g-m-1) p+\left(\pi^{\prime}\right)^{*}\left(\omega_{2}^{\prime}\right)\right)\right) .
$$

В самом деле, тип $\pi^{\prime}$ равен $\nu=(b+1, b, 1,1)$ или $(b, b+1,1,1), \Gamma$ имеет единственную точку Вейерштрасса над $\omega_{2}^{\prime}$, обозначаемую через $p_{2}^{\prime}$. Следовательно, дивизор $\left(\pi^{\prime}\right)^{*}\left(\omega_{2}^{\prime}\right)$ линейно эквивалентен $(m-1) p+p_{2}^{\prime}$ и потенциал $u(x)$ ассоциирован с $\left(\widehat{\varphi} \circ \pi^{\prime} ; \mathscr{O}_{\Gamma}\left((g-2) p+p_{2}^{\prime}\right)\right)$. Аналогичная конструкция может быть осуществлена и в случае $2 \rho=\omega_{2}$ или $2 \rho=\omega_{3}$. 


\section{7. Плоская модель для $\mathscr{R}(n, \mu)\left(\mu^{(2)}=2 n-3\right)$}

7.1. Пара функций Вейерштрасса $\left(\wp, \wp^{\prime}\right)$ определяет классическое вложение эллиптической кривой $(X, q)=(\mathbb{C} / \Lambda, 0)$ в проективную плоскость: $\forall z \neq q, z \mapsto$ $\left(\wp(z): \wp^{\prime}(z): 1\right) \in \mathbb{P}^{2}(\mathbb{C})$. Образ $X$ является замьканием гладкой аффинной кубики $\left\{y^{2}=4 \prod_{i=1}^{3}\left(x-e_{i}\right)\right\}$, где $e_{i}=\wp\left(\omega_{i}\right), \sum_{i=1}^{3} e_{i}=0$ и $e_{i} \neq e_{j}$, если $i \neq j$. Непосредственная проверка приводит к следуюшим двум результатам.

7.2. ЛЕммА. Функиии ६ и $\wp^{\prime}$ (ассоциированные с решеткой $\Lambda$ ) удовлетворяют следующим функииональным уравнениям при произвольном $\rho \in X u j \in$ $\{j, k, \ell\}=\{1,2,3\}$ :

$$
\begin{gathered}
\left(\wp(\rho)-e_{j}\right)\left(\wp\left(\rho-\omega_{j}\right)-e_{j}\right)=\left(e_{k}-e_{j}\right)\left(e_{\ell}-e_{j}\right), \\
\wp^{\prime}\left(\rho-\omega_{j}\right)\left(\wp(\rho)-e_{j}\right)^{2}=-\wp^{\prime}(\rho)\left(e_{k}-e_{j}\right)\left(e_{\ell}-e_{j}\right) .
\end{gathered}
$$

7.3. ПРЕДЛОЖЕНИЕ. Для любого $\vec{a}=\left(a_{i}\right) \in \mathbb{N}^{4} u \rho \in X \backslash\left\{\omega_{i}, i=0, \ldots, 3\right\}$ “фундаментальное" уравнение (7) әквивалентно

$\left(2 a_{0}+1\right)^{2} \prod_{i=1}^{3}\left(\wp(\rho)-e_{i}\right)^{2}=\sum_{j=1}^{3}\left(2 a_{j}+1\right)^{2}\left(e_{k}-e_{j}\right)\left(e_{\ell}-e_{j}\right)\left(\wp(\rho)-e_{k}\right)^{2}\left(\wp(\rho)-e_{\ell}\right)^{2}$.

7.4. ОПРЕДЕЛЕнИЕ. Уравнение из п. 7.3 можно интерпретировать как определяющее поверхность степени 6 в проективном пространстве $\left\{\left(e_{0}: e_{1}: e_{2}: e_{3}\right)\right\}=\mathbb{P}^{3}(\mathbb{C})$ $\left(e_{0}:=\wp(\rho)\right)$. Пересечение последнего пространства с гиперплоскостью $\mathbb{H}:=\left\{e_{1}+\right.$ $\left.e_{2}+e_{3}=0\right\} \subset \mathbb{P}^{3}(\mathbb{C})$ является плоской секстикой, обозначаемой через $\mathscr{C}_{\vec{a}}$.

7.5. ЗАмЕчАния. 1) Мы сопоставили каждой паре $(\pi ; \xi)$ такой, что $\pi \in R(n, \mu, X)$, $\mu^{(2)}=2 n-3$ и $\xi \in\left\{\xi_{A, k}, \xi_{B, k}, k=0, \ldots, 3\right\}$ (см. п. 6.3), эллиптический конечно-зонньй потенциал вида (6), а также, целочисленньй вектор $\vec{a}=\left(a_{i}\right)$ и пару точек $\{\rho,-\rho\}$, удовлетворяюших (7). Другими словами, каждая введенная вьше $\theta$-характеристика $\xi$ определяет инъективное отображение (п. 1.9) $R(n, \mu, X)$ в $\mathscr{C}_{\vec{a}}$, где $\vec{a}=A^{k}(\mu)$ (соответственно $\left.\vec{a}=B^{k}(\mu)\right)$, если $\xi=\xi_{A, k}\left(\right.$ соответственно $\left.\xi=\xi_{B, k}\right)$.

$2)$ Деформация решетки $\Lambda, \Lambda^{\prime}=\alpha \Lambda\left(\alpha \in \mathbb{C}^{*}\right)$, приводит к изменению локальной координаты с $z$ на $\alpha z$. Отсюда немедленно следует, что соответствующая ю-функция Вейерштрасса, а также ее значения $\left\{e_{i}=\wp\left(\omega_{i}\right), i=1,2,3\right\}$ (соответственно $\wp^{\prime}$ ) умножаются на $\alpha^{2}$ (соответственно на $\alpha^{3}$ ). Преобразованная эллиптическая кривая, $X^{\prime}=\mathbb{C} / \Lambda^{\prime}$, изоморфна исходной. Следовательно, $R\left(n, \mu, X^{\prime}\right)$ равно $R(n, \mu, X)$ и отображается в то же множество точек $\mathscr{C}_{\vec{a}}$ (п. 1.12).

7.6. ОПРЕДЕЛЕНИЕ. Обозначим через $\mathscr{P}$ рациональную проекцию точки $(1: 0$ : $0: 0) \in \mathbb{H} \simeq \mathbb{P}^{2}(\mathbb{C})$ в проективную прямую $\left\{e_{0}=0\right\} \subset \mathbb{H}$ и через $\mathfrak{X}-$ пространство модулей всех эллиптических кривых, фиксированных заданием их полупериодов. Для любого $\vec{a} \in \mathbb{N}^{4}$ ограничение $\mathscr{P}$ на $\mathscr{C}_{\vec{a}} \backslash \bigcup_{j=1}^{3} \Delta_{j}$ (где $\Delta_{j}=\left\{e_{k}-e_{\ell}=0\right\}$ ) определяет конечньй морфизм степени 6 на $\left\{e_{0}=0\right\} \backslash \bigcup_{j=1}^{3} \Delta_{j}$ (проективной прямой минус три точки), которая изоморфна $\mathfrak{X}$. Рассмотрим, с другой стороны, одномерное алгебраическое семейство

$$
\mathscr{R}(n, \mu):=\bigcup_{X \in \mathfrak{X}} R(n, \mu, X) .
$$


Мыможем расширить (восемь) инъективных отображений $R(n, \mu, X) \rightarrow \mathscr{C}_{\vec{a}} \backslash \bigcup_{j=1}^{3} \Delta_{j}$, определенных в п. 7.5.1) (при $n=\frac{1}{2}\left(\mu^{(2)}+3\right)$ ), до инъективных морфизмов $\mathscr{R}(n, \mu) \rightarrow$ $\mathscr{C}_{\vec{a}} \backslash \bigcup_{j=1}^{3} \Delta_{j}$, обозначаемых соответственно $\mathscr{A}^{k}$ и $\mathscr{B}^{k}(k=0, \ldots, 3)$. Можно также проверить, что все $\mathscr{C}_{\vec{a}}$ пересекают кубику $\bigcup_{j=1}^{3} \Delta_{j}$ в трех точках

$$
O_{1}:=(1:-2: 1: 1), \quad O_{2}:=(1: 1:-2: 1) \text { и } O_{3}:=(1: 1: 1:-2) \text {. }
$$

В частности,

$$
\mathscr{C}_{\vec{a}} \backslash \bigcup_{j=1}^{3} \Delta_{j}=\mathscr{C}_{\vec{a}} \backslash\left\{O_{1}, O_{2}, O_{3}\right\} .
$$

7.7. ПреДлОЖЕнИЕ. Для любого $k=0, \ldots, 3 u \mu \in \mathbb{M}$ фиксируем $n:=\frac{1}{2}\left(\mu^{(2)}+3\right)$ и $\vec{a}:=A^{k}(\mu)$ (соответственно $\vec{a}:=B^{k}(\mu)$ ). Тогда морфизм $\mathscr{A}^{k}: \mathscr{R}(n, \mu) \rightarrow \mathscr{C}_{\vec{a}} \backslash$ $\left\{\mathrm{O}_{1}, \mathrm{O}_{2}, \mathrm{O}_{3}\right\}$ (соответственно $\mathscr{B}^{k}: \mathscr{R}(n, \mu) \rightarrow \mathscr{C}_{\vec{a}} \backslash\left\{\mathrm{O}_{1}, \mathrm{O}_{2}, \mathrm{O}_{3}\right\}$ ) является собственным. $В$ частности, образ $\mathscr{R}(n, \mu)$ является замкнутыцм подмногообразием в $\mathscr{C}_{\vec{a}} \backslash\left\{O_{1}, O_{2}, O_{3}\right\}$.

ДоКАЗАТЕЛЬСТВО. Композиция одного из описанных морфизмов с рациональной проекцией $\mathscr{P}: \mathbb{H} \rightarrow\left\{e_{0}=0\right\}$ (п. 7.6), позволяет восстановить каноническое квазиконечное отображение $\mathscr{R}(n, \mu) \rightarrow \mathfrak{X}$. У тверждение о собственности $\mathscr{A}^{k}$ и $\mathscr{B}^{k}(k=$ $0, \ldots, 3)$ выводится из собственности $\mathscr{R}(n, \mu) \rightarrow \mathfrak{X}$. Действительно, пусть $W(n, \nu, X)$ обозначает несвязное объединение компактифицированных якобианов всех элементов конечного множества $R(n, \nu, X)\left(\nu^{(2)} \leqslant 2 n+1\right)$, и пусть $W(n, X)$ обозначает несвязное объединение

$$
W(n, X):=\coprod_{\nu^{(2)} \leqslant 2 n+1} W(n, \nu, X) .
$$

Бирациональньй инъективньй морфизм, построенный в п. 1.12 и обозначенньй через $I_{n}(X)$, отображает $W(n, X)$ на $\operatorname{KdV}(n, X)$ - замкнутое подмногообразие $n$-х симметрических степеней $X_{n}$, определяемое уравнениями (3).

Варьируя $X$ вдоль всего $\mathfrak{X}$, мы получаем

$$
\mathscr{W}(n, \nu):=\bigcup_{\mathfrak{X}} W(n, \nu, X),
$$

- алгебраическое семейство компактифицированных якобианов $\mathscr{R}(n, \nu)$, так же как и несвязное объединение

$$
\mathscr{W}(n):=\coprod_{\nu^{(2)} \leqslant 2 n+1} \mathscr{W}(n, \nu)
$$

Рассмотрим также универсальную эллиптическую кривую $\overline{\mathfrak{X}} \rightarrow \mathfrak{X}$, параметризованную соответствуюшими функциями Вейерштрасса, обозначенными ю и $\wp^{\prime}$, и пусть $(\overline{\mathfrak{X}} / \mathfrak{X})_{n}$ обозначает их $n$-ю симметрическую степень. Очевидно, что семейство

$$
\mathscr{K} d \mathscr{V}(n):=\bigcup_{\mathfrak{X}} \operatorname{KdV}_{n}(X)
$$


образует замкнутоеподмногообразие в $(\overline{\mathfrak{X}} / \mathfrak{X})_{n}$, которое может быть определено в терминах $\wp^{\prime}$, как и в $(3)$. В частности, естественная проекция $\mathscr{K} d \mathscr{V}(n) \rightarrow \mathfrak{X}$ является собственной и $\mathscr{K} d \mathscr{V}(n)$ распадается на несвязное объединение:

$$
\mathscr{K} d \mathscr{V}(n)=\coprod_{\nu^{(2)} \leqslant 2 n+1} \mathscr{I}_{n}(\mathscr{W}(n, \nu))
$$

где $\mathscr{I}_{n}: \mathscr{W}(n) \rightarrow \mathscr{K} d \mathscr{V}(n)$ глобализует морфизм $I_{n}(X): W(n, X) \rightarrow \operatorname{KdV}(n, X)$ определенньй в п. 1.12. Выбирая такой тип $\mu \in \mathbb{M}$, что $\mu^{(2)}=2 n-3$, и каноническую $\theta$-характеристику, как и в п. 7.5, мы получаем естественное сечение $\mathscr{R}(n, \mu) \rightarrow \mathscr{W}(n, \mu) \subset \mathscr{W}(n)$, которое в композиции с $\mathscr{I}_{n}$ приводит к замкнутому (собственному) инъективному отображению $\mathscr{R}(n, \mu) \rightarrow \mathscr{K} d \mathscr{V}(n)$. Следовательно, проекция $\mathscr{R}(n, \mu) \rightarrow \mathscr{K} d \mathscr{V}(n) \rightarrow \mathfrak{X}$ является собственной.

7.8. ЗАмЕчАнИя. 1) Для того чтобы обобщить теорему п. 6.5 (т.е. найти все эллиптические конечно-зонные потенциалы вида (6)), необходимо рассмотреть все множество пространств модулей $\left\{\mathscr{R}(n, \mu), n=\frac{1}{2}\left(\mu^{(2)}+3\right)\right\}$. На самом деле, мы докажем, что для любого $\vec{a}=\left(a_{i}\right) \in \mathbb{N}^{4}$ сушествуют $(n, \mu)$ и морфизм над $\mathfrak{X}$, $\mathscr{R}(n, \mu) \rightarrow \mathscr{C}_{\vec{a}} \backslash\left\{O_{1}, O_{2}, O_{3}\right\}$ (см. п. 7.6), определяюший изоморфизм между $\mathscr{R}(n, \mu)$ и неприводимой компонентой $\mathscr{C}_{\vec{a}} \backslash\left\{O_{1}, O_{2}, O_{3}\right\}$. В частности, степень естественной проекции $\mathscr{R}(n, \mu) \rightarrow \mathfrak{X}$ будет равна 6 , если и только если $\mathscr{C}_{\vec{a}}-$ неприводимая секстика. Напомним, что, с другой стороны, степень $\mathscr{R}(n, \mu) \rightarrow \mathfrak{X}$ ограничена числом 4 , если $\#\left\{i, \mu_{i}=0\right\} \geqslant 1$. Таким образом, для любого такого $\mu$ и $\vec{a} \in\left\{A^{k}(\mu), B^{k}(\mu), k=0, \ldots, 3\right\}$ секстика $\mathscr{C}_{\vec{a}}$ должна быть приводимой. Наконец, реализуя точное отображение некоторой $\mathscr{C}_{\vec{a}}\left(\vec{a} \in \mathbb{N}^{4}\right)$ в подходящем пучке эллиптических кривых, мы получаем характеризацию множества $\left\{\vec{a} \in \mathbb{N}^{4}, \mathscr{C}_{\vec{a}}\right.$ приводима $\}$ (п. 8.6).

2) Следуюший результат выводится из (п. 9.2): для любого $\vec{a}=\left(a_{i}\right) \in \mathbb{N}^{4}$ такого, что $\left\{2 a_{i}+1, i=0, \ldots, 3\right\}$ линейно независим над $\mathbb{Z}^{*}=\{1,-1\}$, и $X \in \mathfrak{X}$ функция (6) является эллиптическим конечно-зоннымпотенциалом, ассоциированным с некоторыми данньми $(\pi ; \xi)$ (см. п. 6.3), если и только если $\rho \in X \backslash\left\{\omega_{i}\right\}$ удовлетворяет “фундаментальному" уравнению (7).

3) Множество чисел $\left\{2 a_{i}+1, i=0, \ldots, 3\right\}$ является линейно зависимым над $\mathbb{Z}^{*}=$ $\{1,-1\}$ тогда и только тогда, когда $\mathscr{C}_{\vec{a}}$ приводима (п. 8.6). В последнем случае (самое большее) одна из неприводимых компонент $\mathscr{C}_{\vec{a}}$ соответствует (некоторому) $\mathscr{R}(n, \mu)$. Другие компоненты также приводят к эллиптическим конечно-зонньп потенщиалам, хотя они связаны с исключительными накрытиями (см. п. 9.6.4)). Другими словами, для любых $\left(a_{i}\right) \in \mathbb{N}^{4}$ и любой эллиптической кривой $(X, q)$ функция $(6)$ - эллиптический конечно-зонный потенциал тогда и только тогда, когда выполняется условие (7) при $2 \rho \neq 0$.

4) Наконец, зная неприводимое разложение некоторой $\mathscr{C}_{\vec{a}}$, мы в можем улучшить оценки п. 4.12, вычисляя \# $R(n, \mu, X)$ для общей эллиптической кривой $X=X_{\text {gen }}$. Свойство п. 9.6.2) может быть перефразировано как утверждение о том, что $\# R\left(n, \mu, X_{\text {gen }}\right)=6-2 j_{0}$, где $j_{0}$ обозначает число нулевых координат в $\left(\mu_{i}\right)$ (т.е. $\left.j_{0}=\#\left\{i, \mu_{i}=0\right\}\right)$. 


\section{8. Подход эллиптической поверхности в изучении плоской модели $\mathscr{C}_{\vec{a}}$}

Отождествим гиперплоскость $\mathbb{H}=\left\{e_{1}+e_{2}+e_{3}=0\right\} \subset \mathbb{P}^{3}(\mathbb{C})$ с проективной плоскостью $\mathbb{P}^{2}(\mathbb{C})=\left\{\left(e_{0}: e_{1}: e_{2}\right)\right\}$ и обозначим через $O_{1}, O_{2}$ и $O_{3}$ соответственно точки $(1:-2: 1),(1: 1:-2)$ и $(1: 1: 1)$ гиперплоскости $\mathbb{H}$ (или точки $(-2,1),(1,-2)$ и $(1,1)$ аффинной плоскости $\left.\left\{e_{0} \neq 0\right\} \subset \mathbb{H}\right)$.

8.1. Лемма. Для любого $\vec{a} \in \mathbb{N}^{4}$ секстика $\mathscr{C}_{\vec{a}} \subset \mathbb{H}$ (п. 7.4) имеет особенность порядка 3 в точках $\left\{O_{1}, O_{2}, O_{3}\right\}$, и ее точное отображение $\varphi: \widehat{\mathbb{H}} \rightarrow \mathbb{H}$, раздутие $\left\{\mathrm{O}_{1}, \mathrm{O}_{2}, \mathrm{O}_{3}\right\}$, является связныцм дивизором.

ДокАЗАТЕЛЬСтво. Обозначим через $P(\alpha, \beta, \gamma)$ многочлен $(\beta-\alpha)(\gamma-\alpha)\left(e_{0}-\beta\right)^{2} \times$ $\left(e_{0}-\gamma\right)^{2}$. Тогда для любого $\vec{a} \in \mathbb{N}^{4}$ секстика $\mathscr{C}_{\vec{a}}$ определена множеством нулей следуюшего однородного многочлена:

$$
F_{\vec{a}}=\left(2 a_{0}+1\right)^{2} \prod_{j=1}^{3}\left(e_{0}-e_{j}\right)^{2}-\sum_{i=1}^{3}\left(2 a_{i}+1\right)^{2} P\left(e_{i}, e_{k}, e_{\ell}\right) \quad(\{i, k, \ell\}=\{1,2,3\}) .
$$

Заменяя $e_{1}, e_{2}$ и е ез величинами $x:=\frac{e_{1}}{e_{0}}, y:=\frac{e_{2}}{e_{0}}$ и $-(x+y):=\frac{e_{3}}{e_{0}}$ соответственно, мы получаем многочлен, определяющий аффинную секстику $\mathscr{C}_{\vec{a}} \cap\left\{e_{0} \neq 0\right\}$. Непосредственное вычисление показывает, что $\mathscr{C}_{\vec{a}}$ имеет особенности порядка 3 в $\left\{O_{1}, O_{2}, O_{3}\right\}$. При этом соответствуюшие касательные конусы определяются следующими уравнениями $\left(\alpha_{j}:=2 a_{j}+1, j=1,2,3\right)$ :

касательньй конус в $O_{3}=(1,1)$ :

$\left\{[x-y]\left[\alpha_{2} x-\alpha_{1} y+\alpha_{1}-\alpha_{2}\right]\left[\alpha_{2} x+\alpha_{1} y-\alpha_{1}-\alpha_{2}\right]=0\right\} ;$

касательньй конус в $O_{2}=(1,-2)$ :

$\left\{[2 x+y]\left[\left(\alpha_{1}-\alpha_{3}\right) x+\alpha_{1}, y+\alpha_{1}+\alpha_{3}\right]\left[\left(\alpha_{1}+\alpha_{3}\right) x+\alpha_{1}, y+\alpha_{1}-\alpha_{3}\right]=0\right\} ;$

касательньй конус в $O_{1}=(-2,1)$ :

$\left\{[x+2 y]\left[\alpha_{2} x+\left(\alpha_{2}+\alpha_{3}\right) y+\alpha_{2}-\alpha_{3}\right]\left[\alpha_{2} x+\left(\alpha_{2}-\alpha_{3}\right) y+\alpha_{2}+\alpha_{3}\right]=0\right\}$.

Предположим, что $\mathscr{C}_{\vec{a}}$ раскладывается как $\mathscr{C}_{\vec{a}}=\mathscr{C}^{\prime} \cup \mathscr{C}^{\prime \prime}$, и обозначим через $m_{1}^{\prime}, m_{2}^{\prime}$ и $m_{3}^{\prime}$ порядки $\mathscr{C}^{\prime}$ в $O_{1}, O_{2}$ и $O_{3}$ соответственно. Известно, что $6=\operatorname{deg} \mathscr{C}_{\vec{a}}=\operatorname{deg} \mathscr{C}^{\prime}+$ $\operatorname{deg} \mathscr{C}^{\prime \prime}$. Следовательно, $\mathscr{C}^{\prime}$ и $\mathscr{C}^{\prime \prime}$ пересекаются с кратностью $5=5 \cdot 1,8=4 \cdot 2$ или $9=3 \cdot 3 . \mathrm{C}$ другой стороны, их точные отображения при действии $\varphi: \widehat{\mathbb{H}} \rightarrow \mathbb{H}$, обозначенные, как обычно, через $\widehat{\mathscr{C}}^{\prime}$ и $\widehat{\mathscr{C}}^{\prime \prime}$, пересекаются с кратностью

$$
\widehat{\mathscr{C}}^{\prime} \cdot \widehat{\mathscr{C}}^{\prime \prime}=\mathscr{C}^{\prime} \cdot \mathscr{C}^{\prime \prime}-m_{1}^{\prime}\left(3-m_{1}^{\prime}\right)-m_{2}^{\prime}\left(3-m_{2}^{\prime}\right)-m_{3}^{\prime}\left(3-m_{3}^{\prime}\right),
$$

которая, как это легко увидеть, не равна нулю, поскольку $0 \leqslant m_{i}^{\prime} \leqslant 3$ и $m_{i}^{\prime}\left(3-m_{i}^{\prime}\right)=0$ или $2(i=1,2,3)$. Таким образом, $\widehat{\mathscr{C}}^{\prime} \cap \widehat{\mathscr{C}}^{\prime \prime} \neq \varnothing$.

8.2. ЛЕмма. Выберем некоторые $\left(a_{1}, a_{2}, a_{3}\right) \in \mathbb{N}^{3}$ и обозначим $\widehat{\mathscr{C}}_{\left(-\frac{1}{2}, a_{1}, a_{2}, a_{3}\right)} u$ $\widehat{\mathscr{C}}_{0}$ соответственно точные отображения секстик $\mathscr{C}_{\left(-\frac{1}{2}, a_{1}, a_{2}, a_{3}\right)}$ и $\mathscr{C}_{\left(0,-\frac{1}{2},-\frac{1}{2}-\frac{1}{2}\right)}$ при раздутии $\varphi: \widehat{\mathbb{H}} \rightarrow \mathbb{H}$ точек $\left\{O_{1}, O_{2}, O_{3}\right\}$. Пусть $E_{j}(j=1,2,3)$ обозначает исключительную кривую $\varphi^{*}\left(O_{j}\right)$. Тогда $\widehat{\mathscr{C}}_{\left(-\frac{1}{2}, a_{1}, a_{2}, a_{3}\right)}$ несвязно с $\widehat{\mathscr{C}}_{0}$ и линейно эквивалентно $\widehat{\mathscr{C}}_{0}+E_{1}+E_{2}+E_{3}$. 
ДокАЗАТЕЛЬство. Как следует из уравнений (29), касательные конусы секстик $\mathscr{C}_{\left(-\frac{1}{2}, a_{1}, a_{2}, a_{3}\right)}$ и $\mathscr{C}_{\left(0,-\frac{1}{2},-\frac{1}{2}-\frac{1}{2}\right)}$ в $O_{1}, O_{2}$ и $O_{3}$ трансверсальны и имеют порядки 3 и 4 соответственно. Следовательно, их точные отображения не пересекаются (поскольку $\left.\widehat{\mathscr{C}}_{\left(-\frac{1}{2}, a_{1}, a_{2}, a_{3}\right)} \cdot \widehat{\mathscr{C}}_{0}=\mathscr{C}_{\left(-\frac{1}{2}, a_{1}, a_{2}, a_{3}\right)} \cdot \mathscr{C}_{\left(0,-\frac{1}{2},-\frac{1}{2}-\frac{1}{2}\right)}-3 \cdot(3 \cdot 4)=36-36=0\right)$ и

$$
\begin{aligned}
\widehat{\mathscr{C}}_{\left(-\frac{1}{2}, a_{1}, a_{2}, a_{3}\right)} & \equiv \varphi^{*}\left(\mathscr{C}_{\left(-\frac{1}{2}, a_{1}, a_{2}, a_{3}\right)}\right)-3 \sum_{j=1}^{3} E_{j} \\
& \equiv \varphi^{*}\left(\mathscr{C}_{\left(0,-\frac{1}{2},-\frac{1}{2}-\frac{1}{2}\right)}\right)-3 \sum_{j=1}^{3} E_{j} \equiv \widehat{\mathscr{C}}_{0}+\sum_{j=1}^{3} E_{j} .
\end{aligned}
$$

8.3. ПРЕДЛОЖЕНИЕ. Для любых $\left(a_{1}, a_{2}, a_{3}\right) \in \mathbb{N}^{3}$ дивизорьи $\widehat{\mathscr{C}}_{\left(-\frac{1}{2}, a_{1}, a_{2}, a_{3}\right)} u$ $\widehat{\mathscr{C}}_{0}+\sum_{i=1}^{3} E_{i}$ на $\widehat{\mathbb{H}}($ см. n. 8.2) образуют пучок общих гладких неприводимых кривых рода 1. При этом, раздувая девять (возможнно, инфинитезимальньх) базисных точек этого пучка, получаем рациональное эллиптическое расслоение $(\widehat{\mathbb{H}} \leftarrow) \widehat{\mathbb{H}} \rightarrow \mathbb{P}^{1}(\mathbb{C})$ с характеристикой Эйлера-Пуаккаре $\chi(\widehat{\widehat{\mathbb{H}}})=15$.

ДокАЗАТЕльСТво. Вьше было показано, что для любых $\lambda \in \mathbb{C}$ и $\vec{a}=\left(\lambda, a_{1}, a_{2}, a_{3}\right)$ $\in \mathbb{C} \times \mathbb{N}^{3} \mathscr{C}_{\vec{a}}$-плоская секстика арифметического рода $1+\frac{1}{2} \cdot 6 \cdot(6-3)=10$, имеющая особые точки порядка 3 в $O_{1}, O_{2}$ и $O_{3}$. Следовательно, ее точное отображение $\left(\widehat{\mathscr{C}}_{\vec{a}}\right)$ в $\widehat{\mathbb{H}}$ имеет арифметический род 1 и является связным дивизором (п. 8.1). С другой стороны, поскольку $\mathscr{C}_{\left(-\frac{1}{2}, a_{1}, a_{2}, a_{3}\right)}$ и $\mathscr{C}_{\left(0,-\frac{1}{2},-\frac{1}{2},-\frac{1}{2}\right)}$ трансверсальны в $O_{1}, O_{2}$ и $O_{3}$, легко проверяется, что $\widehat{\mathscr{C}}_{\left(-\frac{1}{2}, a_{1}, a_{2}, a_{3}\right)}$ пересекает $\widehat{\mathscr{C}}_{0}+E_{1}+E_{2}+E_{3}$ в гладких точках упомянутого дивизора. В частности, теорема Бертини утверждает, что обший элемент соответствуюшего пучка является гладким и связным (см. п. 8.1), а следовательно, также и неприводимым. ЕР-характеристика $\widehat{\mathbb{H}}$ равна $\chi(\widehat{\mathbb{H}})=\chi\left(\mathbb{P}^{2}(\mathbb{C})\right)+3=6$. Раздувая девять базисных точек пучка, мы в результате получаем рациональную эллиптическую поверхность, $\widehat{\widehat{H}} \rightarrow \mathbb{P}^{1}(\mathbb{C})$, с ЕР-характеристикой $\chi(\widehat{\widehat{H}})=\chi(\widehat{\mathbb{H}})+9=15$. Например, если все $a_{j}(j=1,2,3)$ различны, то $\widehat{\mathscr{C}}_{\left(-\frac{1}{2}, a_{1}, a_{2}, a_{3}\right)}$ трансверсально к $\widehat{\mathscr{C}}_{0}+E_{1}+E_{2}+E_{3}$, и достаточно раздуть девять точек $\widehat{\mathscr{C}}_{\left(-\frac{1}{2}, a_{1}, a_{2}, a_{3}\right)} \cap\left(E_{1} \cup E_{2} \cup E_{3}\right)$.

8.4. ЗАмЕчАниЕ. В соответствии с так назьваемой “формулой Леффшеца" [23; с. 509], ЕР-характеристика всех сингулярных слоев любого такого пучка (в $\widehat{\widehat{H}})$ равна $\chi(\hat{\widehat{H}})=15$. Знание нескольких приводимых слоев (а также легко оцениваемой их ЕР-характеристики) позволяет, в результате, выделить гладкие и неприводимые слои (пा. 8.5 и 8.6).

8.5. Лемма. Для любого $\vec{a} \in \mathbb{N}^{4}$ такого, что $\left\{2 a_{i}+1, i=0, \ldots, 3\right\}$ линейно зависимы над $\mathbb{Z}^{*}=\{1,-1\}$, секстика $\mathscr{C}_{\vec{a}}=\left\{F_{\vec{a}}\left(e_{0}, e_{1}, e_{2}\right)=0\right\}$ (см. (29)) содержит неприводимую конику, проходящую через $\mathrm{O}_{1}, \mathrm{O}_{2}$ и $\mathrm{O}_{3}$, и $\widehat{\mathscr{C}}_{\vec{a}}$ - его точное отобрахение в $\widehat{\widehat{\mathbb{H}}}$, имеет EP-характеристику не меньшую, чем $\chi\left(\widehat{\widehat{\mathscr{C}}}_{\vec{a}}\right) \geqslant 2$. Более 
точно, обозначая $2 a_{i}+1$ через $\alpha_{i}(i=0, \ldots, 3)$, можсно легко проверить, что:

I) если $\alpha_{0}=\alpha_{1}+\alpha_{2}+\alpha_{3}$, то $F_{\vec{a}}\left(e_{0}, e_{1}, e_{2}\right)$ кратно

$$
\begin{aligned}
R_{\vec{a}}^{+++}:= & \alpha_{2} e_{1}^{2}+\left(\alpha_{1}+\alpha_{2}-\alpha_{3}\right) e_{1} e_{2}+\alpha_{1} e_{2}^{2} \\
& +\left(\alpha_{3}-\alpha_{1}\right) e_{0} e_{1}+\left(\alpha_{3}-\alpha_{2}\right) e_{0} e_{2}-\left(\alpha_{1}+\alpha_{2}+\alpha_{3}\right) e_{0}^{2} ;
\end{aligned}
$$

II) если $\alpha_{0}=\left|-\alpha_{1}+\alpha_{2}+\alpha_{3}\right|$, то $F_{\vec{a}}\left(e_{0}, e_{1}, e_{2}\right)$ кратно

$$
\begin{aligned}
R_{\vec{a}}^{-++}:=\alpha_{2} e_{1}^{2}+\left(\alpha_{2}-\alpha_{3}-\alpha_{1}\right) e_{1} e_{2}-\alpha_{1} e_{2}^{2} \\
\quad+\left(\alpha_{1}+\alpha_{3}\right) e_{1} e_{0}+\left(\alpha_{3}-\alpha_{2}\right) e_{0} e_{2}-\left(\alpha_{2}+\alpha_{3}-\alpha_{1}\right) e_{0}^{2}
\end{aligned}
$$

III) если $\alpha_{0}=\left|\alpha_{1}-\alpha_{2}+\alpha_{3}\right|$, то $F_{\vec{a}}\left(e_{0}, e_{1}, e_{2}\right)$ кратно

$$
\begin{aligned}
R_{\vec{a}}^{+-+}:=\alpha_{2} e_{1}^{2}+\left(\alpha_{2}+\alpha_{3}-\alpha_{1}\right) e_{1} e_{2}-\alpha_{1} e_{2}^{2} \\
\quad+\left(\alpha_{1}-\alpha_{3}\right) e_{1} e_{0}-\left(\alpha_{2}+\alpha_{3}\right) e_{0} e_{2}+\left(\alpha_{1}-\alpha_{2}+\alpha_{3}\right) e_{0}^{2}
\end{aligned}
$$

IV) если $\alpha_{0}=\left|\alpha_{1}+\alpha_{2}-\alpha_{3}\right|$, то $F_{\vec{a}}\left(e_{0}, e_{1}, e_{2}\right)$ кратно

$$
\begin{aligned}
& R_{\vec{a}}^{++-}:=\alpha_{2} e_{1}^{2}+\left(\alpha_{1}+\alpha_{2}+\alpha_{3}\right) e_{1} e_{2}+\alpha_{1} e_{2}^{2} \\
&-\left(\alpha_{1}+\alpha_{3}\right) e_{1} e_{0}-\left(\alpha_{2}+\alpha_{3}\right) e_{0} e_{2}-\left(\alpha_{1}+\alpha_{2}-\alpha_{3}\right) e_{0}^{2}
\end{aligned}
$$

ДокАЗАТЕльство. Каждая коника $\{R=0\} \subset \mathbb{H}\left(R=R_{\vec{a}}^{+++}, R_{\vec{a}}^{++-}, R_{\vec{a}}^{+-+}\right.$или $R_{\vec{a}}^{-++}$) является гладкой касательной к $\mathscr{C}_{\vec{a}}$ в $O_{1}, O_{2}$ и $O_{3}$. В частности, сумма кратностей пересечения между $\{R=0\} \cap \mathscr{C}_{\vec{a}}$ в $\left\{O_{1}, O_{2}, O_{3}\right\}$ составляет не менее 12 . Но $\{R=0\} \cdot \mathscr{C}_{\vec{a}}=2 \cdot 6=12$. Следовательно, $\{R=0\} \cap \mathscr{C}_{\vec{a}}$ равно $\left\{O_{1}, O_{2}, O_{3}\right\}$, если $\{R=0\} \subset \mathscr{C}_{\vec{a}}$. С другой стороны, можно легко проверить, что в каждом из перечисленных вьше случаев I)-IV) $R\left(0, e_{1}, e_{2}\right)$ делит $F_{\vec{a}}\left(0, e_{1}, e_{2}\right)$. Другими словами, $\{R=0\} \cap \mathscr{C}_{\vec{a}} \cap\left\{e_{0}=0\right\} \neq \varnothing$. Следовательно, $\mathscr{C}_{\vec{a}}$ содержит конус $\{R=0\}$. Пусть $\mathscr{C}_{\vec{a}}=\{R=0\} \cup\{T=0\}-$ соответствующее разложение. После раздутия $\left(O_{1}, O_{2}\right.$ и $\left.O_{3}\right)$ точные отображения $\{R=0\}$ и $\{T=0\}$ продолжают пересекаться с кратностью 2. Отсюда следует, что ЕР-характеристика $\widehat{\mathscr{C}}_{\vec{a}}$ уже $\geqslant 2$ и может только возрасти после второй серии раздутий. Таким образом, мы доказали, что $\chi\left(\widehat{\hat{\mathscr{C}}}_{\vec{a}}\right) \geqslant 2$.

8.6. ПРЕДЛОЖЕНИЕ. Для любых $\left(a_{1}, a_{2}, a_{3}\right) \in \mathbb{N}^{3}$ обозначим через $\widehat{\widehat{\mathbb{H}}} \rightarrow \mathbb{P}^{1}(\mathbb{C})$ рачиональные эллиптические расслоения, построенные в $n .8 .3$, и через $\widehat{\widehat{\mathscr{C}}}_{\left(-\frac{1}{2}, a_{1}, a_{2}, a_{3}\right)}-$ точное отображсение $\mathscr{C}_{\left(-\frac{1}{2}, a_{1}, a_{2}, a_{3}\right)}$ в $\widehat{\widehat{\mathbb{H}}}$. Тогда это расслоение имеет $m:=2+\#\left\{a_{1}, a_{2}, a_{3}\right\}$ приводимых слоев, c EP-характеристикой, доходящей до $14, u \widehat{\mathscr{C}}_{\left(-\frac{1}{2}, a_{1}, a_{2}, a_{3}\right)}$ в качестве единственного неприводимого слоя с узлом. Все остальные слои являются гладкими и неприводимыми. 
ДокАЗАТЕльство. Вьше было показано, что ЕР-характеристика всех сингулярных слоев $\widehat{\widehat{\mathbb{H}}} \rightarrow \mathbb{P}^{1}(\mathbb{C})$ дорастает до $\chi(\widehat{\widehat{\mathbb{H}}})=15$, и простое вычисление показьвает, что секстика $\mathscr{C}_{\left(-\frac{1}{2}, a_{1}, a_{2}, a_{3}\right)}$ (см. п. 8.2) имеет узел при $(1: 0: 0) \in \mathbb{H}$. В частности, ее точное отображение, $\widehat{\widehat{C}}_{\left(-\frac{1}{2}, a_{1}, a_{2}, a_{3}\right)}$, является либо неприводимой рациональной кривой с узлом $\left(\right.$ и $\left.\chi\left(\widehat{\widehat{\mathscr{C}}}_{\left(-\frac{1}{2}, a_{1}, a_{2}, a_{3}\right)}\right)=1\right)$, либо редуцируемой кривой $\left(и \chi\left(\widehat{\widehat{\mathscr{C}}}_{\left(-\frac{1}{2}, a_{1}, a_{2}, a_{3}\right)}\right) \geqslant 2\right)$. Кроме того, редуцируемьй слой, соответствующий секстике

$$
\mathscr{C}_{\left(0,-\frac{1}{2},-\frac{1}{2},-\frac{1}{2}\right)}=\left\{\prod_{j=1}^{3}\left(e_{0}-e_{j}\right)^{2}=0\right\}\left(\sum_{j=1}^{3} e_{j}=0\right),
$$

изоморфен $\widehat{\mathscr{C}}_{0}+E_{1}+E_{2}+E_{3}$ (см. п. 8.2) и его ЕР-характеристика равна 6. С другой стороны, можно легко проверить, что $\left(m-1=1+\#\left\{a_{1}, a_{2}, a_{3}\right\}\right)$ редуцируемых слоев, полученных в п. 8.5, имеют ЕР-характеристику, равную (по крайней мере) 8. Возникают три случая, подлежащие рассмотрению.

$\#\left\{a_{1}, a_{2}, a_{3}\right\}=3$. Сушествуют четыре различные значения $a_{0}$ такие, что множество $\left\{2 a_{i}+1, i=0, \ldots, 3\right\}$ линейно зависимо над $\mathbb{Z}^{*}=\{1,-1\}$. Следовательно, сушествуют четыре приводимых слоя $\widehat{\widehat{C}}_{\vec{a}}$ с $\chi\left(\widehat{\mathscr{C}}_{\vec{a}}\right) \geqslant 2$ (п. 8.5).

$\#\left\{a_{1}, a_{2}, a_{3}\right\}=2$. Сушествует пара $(a, b) \in \mathbb{N}^{2}(a \neq b)$ такая, что, с точностью до упорядочения, $\left(a_{1}, a_{2}, a_{3}\right)=(a, a, b)$, и только три значения $a_{0}$ такие, что $\left\{2 a_{i}+1, i=0, \ldots, 3\right\}$ линейно зависим над $\mathbb{Z}^{*}$. А именно, $2 a_{0}+1=2 b+1,4 a+$ $3+2 b$ или $|4 a+1-2 b|$. В первом случае $\left(a_{0}=b\right)$ соответствующая редуцируемая секстика $\mathscr{C}_{\vec{a}}=\left\{F_{\vec{a}}=0\right\}$ является объединением трех неприводимых коник, проходяших через $O_{1}, O_{2}$ и $O_{3}$. Из п. 8.5, I) и II), следует, что $F_{\vec{a}}$ факторизуется как $F_{\vec{a}}=F_{(b, a, a, b)}=R_{\vec{a}}^{-++} \cdot R_{\vec{a}}^{+-+} \cdot R$ для некоторого неприводимого многочлена $R:=R_{(b, a, a, b)}($ который может быть легко вьписан). Следовательно,

$$
\mathscr{C}_{\vec{a}}=\left\{R_{\vec{a}}^{-++}=0\right\} \cup\left\{R_{\vec{a}}^{+-+}=0\right\} \cup \mathscr{C o n}_{\vec{a}},
$$

где

$$
\mathscr{C o n}_{\vec{a}}:=\left\{R_{(b, a, a, b)}=0\right\}
$$

Кроме того, последние коники трансверсальны в $O_{1}$ и $O_{2}$, но $\mathscr{C}_{\vec{a}}^{-+}:=\left\{R_{\vec{a}}^{-++}=0\right\}$ касается с $\mathscr{C}_{\vec{a}}^{+-+}:=\left\{R_{\vec{a}}^{+-+}=0\right\}$ и трансверсальна к $\mathscr{C}$ о $n_{\vec{a}}$ в $O_{3}$ (см. (29) при $\alpha_{1}=$ $\left.\alpha_{2} \neq \alpha_{3}\right)$. Поэтому точное отображение $\mathscr{C}_{\vec{a}}$ в $\widehat{\widehat{H}}$ равно

$$
\widehat{\widehat{C}}_{\vec{a}}=\widehat{\widehat{C}}_{\vec{a}}^{-++} \cup \widehat{\widehat{C}}_{\vec{a}}^{+-+} \cup \widehat{\widehat{C}}_{\text {on }} \vec{a}
$$

и $\widehat{\widehat{\mathscr{C}}}_{\vec{a}}^{-++} \cdot \widehat{\widehat{\mathscr{C}}}_{\vec{a}}^{+-+}=0$ (но $\left.\widehat{\widehat{\mathscr{C}}}_{\vec{a}}^{-++} \cdot \widehat{\widehat{\mathscr{C}}}_{\text {о }} n_{\vec{a}}=\widehat{\widehat{\mathscr{C}}}_{\vec{a}}^{+-+} \cdot \widehat{\widehat{\mathscr{C}}}_{\text {оn }} \vec{a}=1\right)$. В частности, $\chi\left(\widehat{\widehat{\mathscr{C}}}_{\vec{a}}\right)=4$. Таким образом, мы получаем три редуцируемые слоя (п. 8.5) с ЕР-характеристикой, равной (по крайней мере) $4+2+2=8$.

$\#\left\{a_{1}, a_{2}, a_{3}\right\}=1$. Поскольку $a_{1}=a_{2}=a_{3}$, лемма п. 8.5 приводит только к двум значениям $a_{0}$ таким, что $\left\{2 a_{i}+1, i=0, \ldots, 3\right\}$ линейно зависимо над $\mathbb{Z}^{*}$. А именно, $a_{0}=a_{1}$ и $a_{0}=3 a_{1}$. Для $a_{0}=a_{1}=a_{2}=a_{3} F_{\vec{a}}$ факторизуется как произведение 
трех неприводимых квадратичных многочленов: $\left\{R_{\vec{a}}^{++-}, R_{\vec{a}}^{+-+}, R_{\vec{a}}^{-++}\right\}$. Другими словами,

$$
\begin{aligned}
F_{\left(a_{0}, a_{0}, a_{0}, a_{0}\right)=} & \left(2 a_{0}+1\right)^{2}\left[e_{1}^{2}+3 e_{1} e_{2}+e_{2}^{2}-2 e_{0} e_{1}-2 e_{0} e_{2}-e_{0}^{2}\right] \\
& \times\left[e_{1}^{2}+e_{1} e_{2}-e_{2}^{2}-2 e_{0} e_{2}+e_{0}^{2}\right]\left[e_{1}^{2}-e_{1} e_{2}-e_{2}^{2}+2 e_{1} e_{0}-e_{0}^{2}\right] .
\end{aligned}
$$

Следовательно,

$$
\mathscr{C}_{\vec{a}}=\left\{R_{\vec{a}}^{++-}=0\right\} \cup\left\{R_{\vec{a}}^{+-+}=0\right\} \cup\left\{R_{\vec{a}}^{-++}=0\right\},
$$

и соответствуюшее точное отображение в $\widehat{\widehat{\mathbb{H}}}$ образовано тремя несвязньми экземплярами $\mathbb{P}^{1}(\mathbb{C})$ и имеет ЕР-характеристику, равную 6 . Таким образом, мы получили два редуцируемых слоя (п. 8.5) с ЕР-характеристикой, равной (по крайней мере) $6+2=8$.

Суммируя все наши аргументы, заключаем, что поскольку $\chi(\widehat{\widehat{\mathbb{H}}})=15$, то $1+\#\left\{a_{1}, a_{2}, a_{3}\right\}$ редуцируемых слоев, полученных в п. 8.5, имеют минимально возможную ЕР-характеристику (т.е. $(2,2,2,2),(4,2,2)$ и $(6,2))$, в то время как $\chi\left(\widehat{\widehat{\mathscr{C}}_{0}}\right)=6$. Следовательно, $\chi\left(\widehat{\widehat{\mathscr{C}}}_{\left(-\frac{1}{2}, a_{1}, a_{2}, a_{3}\right)}\right)=\chi(\widehat{\widehat{\mathbb{H}}})-8-6=1$ (т.е. $\widehat{\widehat{\mathscr{C}}}_{\left(-\frac{1}{2}, a_{1}, a_{2}, a_{3}\right)}$ - рациональная неприводимая кривая, имеюшая узел при $(1: 0: 0))$ и все остальные слои являются гладкими. Другими словами, для любого $\lambda \in \mathbb{C}$ слой $\widehat{\widehat{C}}_{\left(\lambda, a_{1}, a_{2}, a_{3}\right)}$ эллиптическая кривая, если и только если

$(2 \lambda+1)^{2} \notin\left\{0,\left(\alpha_{1}+\alpha_{2}+\alpha_{3}\right)^{2},\left(-\alpha_{1}+\alpha_{2}+\alpha_{3}\right)^{2},\left(\alpha_{1}-\alpha_{2}+\alpha_{3}\right)^{2},\left(\alpha_{1}+\alpha_{2}-\alpha_{3}\right)^{2}\right\}$.

\section{9. Полное описание нового класса четных эллиптических конечно-зонных потенциалов}

9.1. Предложение п. 8.6 и его доказательство основано на подробном изучении подмножества редуцируемых секстик $\left\{\mathscr{C}_{\vec{a}}, \vec{a} \in \mathbb{N}^{4}\right\}$ и позволяет перейти к заключительному этапу описания связи между $\mathscr{R}(n, \mu)\left(\mu^{(2)}=2 n-3\right)$ и $\mathscr{C}_{\vec{a}}$ при любых $\vec{a} \in\left\{A^{k}(\mu), B^{k}(\mu), k=0, \ldots, 3\right\}$ (п. 9.6). Мы используем эту связь для получения полного описания всех четных эллиптических конечно-зонных потенщиалов вида (6), где $\vec{a}=\left(a_{i}\right) \in \mathbb{N}^{4}$ и $2 \rho \neq 0$ (пп. 9.7, 9.9). Напомним, что подмножество всех возможных типов, $\mathbb{M} \subset \mathbb{N}^{4}$, раскладывается в несвязное объединение $\mathbb{M}=\coprod_{j=0}^{3} \mathbb{M} \# j$, где $\mu$ принадлежит $\mathbb{M} \# j$ тогда и только тогда, когда $\mu \in \mathbb{M}$ и \#\{i, $\left.\mu_{i}=0\right\}=j$. При этом для любого $\vec{a} \in \mathbb{N}^{4}$ существует единственный тип $\mu \in \mathbb{M}$ такой, что $\vec{a} \in$ $\left\{A^{k}(\mu), B^{k}(\mu), k=0, \ldots, 3\right\}[41 ; \S \S 4.4,4.5,5.3]$. Обозначим через $n$ натуральное число

$$
n:=\frac{1}{2}\left(\mu^{(2)}+3\right)=2+\frac{1}{2} \sum_{i=0}^{3} a_{i}\left(a_{i}+1\right)
$$

и через $F_{\vec{a}}$ - однородньй многочлен $(28)$ такой, что $\mathscr{C}_{\vec{a}}=\left\{F_{\vec{a}}=0\right\} \cap \mathbb{H}$. Исчерпывающее изучение всех возможностей приводит к рассмотрению четырех различных случаев.

9.2. ТЕОРемА (случай $\mu \in \mathbb{M} \# 0)$. Следующие свойства эквивалентнь:

i) $\mu \in \mathbb{M}^{\# 0}$ (m.e. $\mu_{i} \neq 0$ для всех $\left.i=0, \ldots, 3\right)$; 
ii) множество чельх чисел $\left\{2 a_{i}+1, i=0, \ldots, 3\right\}$ линейно независимо над $\mathbb{Z}^{*}=\{1,-1\}$

iii) инбективное отображение $\mathscr{R}(n, \mu) \rightarrow \mathscr{C}_{\vec{a}} \backslash\left\{O_{1}, O_{2}, O_{3}\right\}$ (cм. n. 7.7) является изоморфизмом;

iv) для любой эллиптической кривой $X$ и любого $\rho \in X \backslash\left\{\omega_{i}\right\}$ четная функция (6) является одним из эллиптических конечно-зонных потенциалов, канонически ассочиированных с некоторым $\pi \in R(n, \mu, X)$ (cм. n. 6.3), если и только если выполняется (7).

ДокАЗАТЕЛЬство. Эквивалентность і) $\Leftrightarrow$ iі) может быть непосредственно проверена с помошью формул для $A^{k}$ и $B^{k}(k=0, \ldots, 3)$ (см., например, п. 6.3 или [41; $\S \S 4.4,5.4 .3])$. Из п. 8.6 следует, что $\mathscr{C}_{\vec{a}} \backslash\left\{O_{1}, O_{2}, O_{3}\right\}$ является гладким и неприводимьм, если и только если $\left\{2 a_{i}+1\right\}$ линейно независимы над $\mathbb{Z}^{*}$. Напомним, что, с другой стороны, образ $\mathscr{R}(n, \mu)$ при инъективном морфизме $\mathscr{A}^{k}$ или $\mathscr{B}^{k}$ образует замкнутое подмногообразие в $\mathscr{C}_{\vec{a}} \backslash\left\{O_{1}, O_{2}, O_{3}\right\}($ п. 7.7) и что степень $(\mathscr{R}(n, \mu) \rightarrow \mathfrak{X})<6$, если $\mu \notin \mathbb{M}^{\# 0}$ (п. 4.12). Поэтому из пп. 9.2.i) и 9.2.ii) следует, что собственное инъективное отображение $\mathscr{R}(n, \mu) \rightarrow \mathscr{C}_{\vec{a}} \backslash\left\{O_{1}, O_{2}, O_{3}\right\}$ должно быть также сюръективным и, следовательно, изоморфизмом, поскольку $\mathscr{C}_{\vec{a}}$ является гладким вне $\left\{O_{1}, O_{2}, O_{3}\right\}$. Наконец, из п. 9.2.ii) с очевидностью следует утверждение п. 9.2.iv), которое, в свою очередь, означает, что $\operatorname{deg}(\mathscr{R}(n, \mu) \rightarrow \mathfrak{X})=6$. Следовательно, $\mu \in \mathbb{M}^{\# 0}$.

9.3. Теорема (случай $\mu \in \mathbb{M} \# 1$ ). Следующие свойства әквивалентны:

i) $\mu \in \mathbb{M}^{\# 1}$ (m.e. $\left.\#\left\{i, \mu_{i}=0\right\}=1\right)$;

ii) множество чисел $\left\{2 a_{i}+1, i=0, \ldots, 3\right\}$ линейно зависимо над $\mathbb{Z}^{*}=$ $\{1,-1\}$, но $\vec{a} \notin\{(c, c, d, d),,(c, d, c, d),(c, d, d, c) ; c, d \in \mathbb{N}\} ;$

iii) однородныи многочлен $F_{\vec{a}}\left(e_{0}, e_{1}, e_{2}\right)$ (см. (28)) факторизуется как $F_{\vec{a}}=$ $T \cdot R$, где $R \in\left\{R_{\vec{a}}^{+++}, R_{\vec{a}}^{-++}, R_{\vec{a}}^{+-+}, R_{\vec{a}}^{++-}\right\}$(cм. n. 8.5). Кроме того, плоская квартика $\mathscr{Q}_{\vec{a}}:=\{T=0\}$, являющаяся неприводимой компонентой $\mathscr{C}_{\vec{a}}$, а также образом инвективного отображения $\mathscr{R}(n, \mu) \rightarrow \mathscr{C}_{\vec{a}} \backslash$ $\left\{O_{1}, O_{2}, O_{3}\right\}$, определяет изоморфизм межуду $\mathscr{R}(n, \mu)$ и $\mathscr{Q}_{\vec{a}} \backslash\left\{O_{1}, O_{2}, O_{3}\right\}$;

iv) $F_{\vec{a}}$ факторизуется так же, как и в $n$. 9.3.iii), и для любой эллиптической кривой $X$ и любого $\rho \in X(2 \rho \neq 0)$ функция (6) - один из әллиптических конечно-зонных потенииалов, канонически ассоциированных с некоторым $\pi \in R(n, \mu, X)$ (см. п. 6.3) тогда и только тогда, когда $T\left(\wp(\rho), e_{1}, e_{2}\right)=0$.

ДокАЗАТЕЛЬСТвО. Эквивалентность свойств і) и іi) следует непосредственно из соответствуюших формул (п. 6.3) и подразумевает, что $F_{\vec{a}}$ факторизуется как в п. 9.3.iii) (см. п. 8.5). Например, если $2 a_{0}+1=\sum_{j=1}^{3}\left(2 a_{j}+1\right)$, то $R_{\vec{a}}^{+++}$делит $F_{\vec{a}}$, $F_{\vec{a}}=R_{\vec{a}}^{+++} T$, и квартика $\mathscr{Q}_{\vec{a}}=\{T=0\}$ неприводима, поскольку

$$
\vec{a} \notin\{(c, c, d, d),(c, d, d, c),(c, d, c, d) ; c, d \in \mathbb{N}\}
$$

(см. п. 8.6). В частности, собственньй морфизм (п. 7.7) $\mathscr{R}(n, \mu) \rightarrow \mathscr{C}_{\vec{a}} \backslash\left\{O_{1}, O_{2}, O_{3}\right\}$ должен накрьвать либо аффинную конику $\{R=0\} \backslash\left\{O_{1}, O_{2}, O_{3}\right\}$, либо аффинную квартику $\mathscr{Q}_{\vec{a}} \backslash\left\{O_{1}, O_{2}, O_{3}\right\}$. Как показано в п. 4.12, для произвольного $X \in \mathfrak{X}$ $\# R(n, \mu, X) \geqslant 2$. Следовательно, $\operatorname{deg}(\mathscr{R}(n, \mu) \rightarrow \mathfrak{X}) \geqslant 2$, причем равенство имеет место, если и только если проекция $\mathscr{R}(n, \mu) \rightarrow \mathfrak{X}$ не разветвлена. С другой стороны, можно легко проверить (используя формулу Гурвица и локальное разложение 
$R\left(e_{0}, e_{1}, e_{2}\right)$ в окрестности $\left.\left\{O_{1}, O_{2}, O_{3}\right\}\right)$, что проекция $\{R=0\} \backslash\left\{O_{1}, O_{2}, O_{3}\right\} \rightarrow \mathfrak{X}$ действительно является разветвленной. Следовательно, $\operatorname{deg}(\mathscr{R}(n, \mu) \rightarrow \mathfrak{X})>2$ и $\mathscr{R}(n, \mu)$ накрьвает аффинную квартику $\mathscr{Q}_{\vec{a}} \backslash\left\{O_{1}, O_{2}, O_{3}\right\}$. Наконец, поскольку $\mathscr{Q}_{\vec{a}}$ является гладким вне $\left\{O_{1}, O_{2}, O_{3}\right\}$, упомянутое инъективное отображение определяет изоморфизм между $\mathscr{R}(n, \mu)$ и $\mathscr{Q}_{\vec{a}} \backslash\left\{O_{1}, O_{2}, O_{3}\right\}$. Свойство п. 9.3.iii) очевидно, приводит к п. 9.3.iv), что, в свою очередь, означает, что $F_{\vec{a}}$ факторизуется и $\operatorname{deg}(\mathscr{R}(n, \mu) \rightarrow \mathfrak{X})=4$. Следовательно, $\#\left\{i, \mu_{i}=0\right\}=1$ (т.е. $\mu \in \mathbb{M}^{\# 1}$ ).

9.4. Теорема (случай $\mu \in \mathbb{M}^{\# 2}$ ). Следующие свойства эквивалентны:

i) $\mu \in \mathbb{M}^{\# 2}$;

ii) существуют $c, d \in \mathbb{N}, c \neq d$, такие, что $\vec{a}$ равен либо $(c, c, d, d)$, либо $(c, d, c, d)$, либо $(c, d, d, c)$;

iii) $\mathscr{C}_{\vec{a}} \backslash\left\{O_{1}, O_{2}, O_{3}\right\}$ распадается на объединение трех неприводимых аффинных коник, и $\mathscr{R}(n, \mu)$ изоморфно отображается на одну из них (обозначенную в n. 8.6 через $\mathscr{C}_{\vec{a}}$ ). Кроме того, пусть $\vec{a}$ такой, как в n. 9.4.ii). Тогда для произвольной әллиптической кривой $X$ и произвольного $\rho \in X$ функция (6) является одним из четных әллиптических конечно-зонных потенциалов, канонически ассоциированных с некоторым $\pi \in R(n, \mu, X)$ (cм. n. 6.3), тогда и только тогда, когда $2 \rho$ равно $\omega_{1}($ при $\vec{a}=(c, c, d, d))$, $\omega_{2}($ при $\vec{a}=(c, d, c, d))$ или $\omega_{3}($ при $\vec{a}=(c, d, d, c))$. В частности, и $(x)-$ непримитивный потенциал ( $n$. 1.13.2)).

ДокАЗАТЕЛЬСтво. Свойства пп. 9.4.i) и 9.4.іi), как легко видеть, эквивалентны и позволяют заключить, что $R(n, \mu, X) \neq \varnothing$ для некоторого $X \in \mathfrak{X}$. Из них также следует, что $\operatorname{deg}(\mathscr{R}(n, \mu) \rightarrow \mathfrak{X}) \leqslant 2$ (п. 4.12$)$ и что $\mathscr{C}_{\vec{a}}$ расщепляется как сумма трех гладких коник (п. 8.6). Следовательно, инъективное отображение $\mathscr{R}(n, \mu) \rightarrow \mathscr{C}_{\vec{a}} \backslash$ $\left\{O_{1}, O_{2}, O_{3}\right\}$ определяет изоморфизм $\mathscr{R}(n, \mu)$ с одной из неприводимых компонент $\mathscr{C}_{\vec{a}} \backslash$ $\left\{O_{1}, O_{2}, O_{3}\right\}$. Обратно, расшепление $\mathscr{C}_{\vec{a}}$ на сумму коник при $\mathscr{R}(n, \mu) \neq \varnothing$ подразумевает, что $\mu \in \mathbb{M}^{\# 2} \cup \mathbb{M}^{\# 3}$, но $\mu \notin \mathbb{M}^{\# 3}$. Следовательно, $\mu \in \mathbb{M}^{\# 2}$. Наконец, докажем, что $R(n, \mu, X) \neq \varnothing$ для любого $X \in \mathfrak{X}$, если $\mu \in \mathbb{M}^{\# 2}$ (т.е. если $\mu$ равно либо $(2 \ell+1,2 k, 0,0)$, либо $(2 \ell+1,0,2 k, 0)$, либо $(2 \ell+1,0,0,2 k)$ при некотором $k, \ell \in \mathbb{N}, k \neq 0)$. Предположим, например, что $\mu=(2 \ell+1,2 k, 0,0)$, следовательно, $n=2\left(\ell^{2}+\ell+k^{2}+1\right)$, и рассмотрим проекцию $r: X \rightarrow Y:=X /\left\langle\omega_{0}, \omega_{1}\right\rangle$. Выберем $\rho \in X$ такое, что $2 \rho=\omega_{1}$. Можно легко проверить, что

$$
\nu_{0}:=r\left(\omega_{0}\right)=r\left(\omega_{1}\right), \quad \nu_{1}:=r\left(\omega_{2}\right)=r\left(\omega_{3}\right), \quad \nu_{2}:=r(\rho), \quad \nu_{3}:=r\left(\rho+\omega_{2}\right)=\nu_{2}+\nu_{1}
$$

являются полупериодами $Y$. При этом дуальный морфизм $\widehat{r}: Y \rightarrow X$ имеет ядро $\operatorname{ker} \widehat{r}=\left\langle\nu_{0}, \nu_{1}\right)$. Рассмотрим исключительные накрытия $\pi^{\prime}: \Gamma^{\prime} \rightarrow Y$ и $\pi^{\prime \prime}: \Gamma^{\prime \prime} \rightarrow Y$, типов

$$
\mu^{\prime}=(\ell+1, \ell, k-1, k+1) \text { и } \mu^{\prime \prime}=(\ell+1, \ell, k+1, k-1)
$$

соответственно, если $\ell \equiv k-1(\bmod 2) ;$ в противном случае выберем

$$
\mu^{\prime}=(\ell, \ell+1, k-1, k+1) \quad \text { и } \mu^{\prime \prime}=(\ell, \ell+1, k+1, k-1) .
$$


Они имеют одинаковые степень, $\ell^{2}+\ell+k^{2}+1=\frac{n}{2}$, и арифметический род $\frac{1}{2}\left(\left(\mu^{\prime}\right)^{(1)}-1\right)$ $=\ell+k=\frac{1}{2}\left(\left(\mu^{\prime \prime}\right)^{(1)}-1\right)$. Композиция $\pi^{\prime}$ и $\pi^{\prime \prime}$ с $\widehat{r}: Y \rightarrow X$ приводит к двум гиперэллиптическим касательньм накрытиям степени $n$ над $X$, типа $\mu=(2 \ell+1,2 k, 0,0)$ и арифметического рода $\ell+k=\frac{1}{2}\left(\mu^{(1)}-1\right)$. Следовательно, $R(n, \mu, X)$ содержит $\left\{\widehat{r} \circ \pi^{\prime}, \widehat{r} \circ \pi^{\prime \prime}\right\}$. Кроме того, композищия проектирования $r: X \rightarrow Y$ с каноническими эллиптическими конечно-зонными потенциалами, ассоциированными с $\pi^{\prime}$ и $\pi^{\prime \prime}$, приводит к потенциалам, ассоциированньм с $\widehat{r} \circ \pi^{\prime}$ и $\widehat{r} \circ \pi^{\prime \prime}$ соответственно. Можно проверить, в частности (упражнение!), что пара непримитивных функций,

$$
\begin{gathered}
u(x)=2 f(x-\tau)+c(c+1) f(x)+d(d+1) f\left(x-\omega_{2}\right) \\
\left(\text { где } 2 \tau=\omega_{1} \text { и } f(x) \equiv \wp(x)+\wp\left(x-\omega_{1}\right)\right),
\end{gathered}
$$

может быть получена именно таким образом. Наконец, хотя это и не утверждалось в п. 9.4, отметим, что для любого $X \in \mathfrak{X} R(n, \mu, X)$ не только содержит, но и равно $\left\{\widehat{r} \circ \pi^{\prime}, \widehat{r} \circ \pi^{\prime \prime}\right\}$. Это следует из того, что $\# R(n, \mu, X) \leqslant 2\left(\right.$ п. 4.12.2)) и что $\widehat{r} \circ \pi^{\prime}$ неизоморфно $\widehat{r} \circ \pi^{\prime \prime}$ (см. п. 4.12).

9.5. Теорема (случай $\mu \in \mathbb{M}^{3}$ ). Следующие свойства әквивалентны:

i) $\mu \in \mathbb{M}^{\# 3}=\{(2 k+1,0,0,0), k \in \mathbb{N}\}$;

ii) $\vec{a} \in\{(b, b, b, b), b \in \mathbb{N}\}$;

iii) $\mathscr{C}_{\vec{a}}$ расщепляется на сумму трех коник и $\mathscr{R}(n, \mu)=\varnothing$.

В последнем случае

$$
\sum_{i=0}^{3}\left(2 a_{i}+1\right)^{2} \wp^{\prime}\left(\rho-\omega_{i}\right)=0
$$

эквивалентно равенству $4 \rho=0$ при $2 \rho \neq 0$. Для любого решения $\rho$ четная функиия

$$
u(x)=2 \wp(x-\rho)+2 \wp(x+\rho)+b(b+1) \sum_{i=0}^{3} \wp\left(x-\omega_{i}\right)
$$

является непримитивным эллиптическим конечно-зонным потенииалом, ассочиированным с исключительным накрытием степени $n:=2\left(b^{2}+b+1\right)$ и типа, равного либо $(2 k+1,2,0,0)$, либо $(2 k+1,0,2,0)$, либо $(2 k+1,0,0,2)$.

ДокАЗАТЕЛЬство. Эквивалентность следует (снова) из пП. 4.12 и 8.6, в то время как последнее утверждение было доказано в п. 6.6 .

Мы достигаем главную цель нашей работы в нижеследуюших трех следствиях. Первое резюмирует глобальные свойства пространств модулей $\{\mathscr{R}(n, \mu), \mu \in \mathbb{M}$, $\left.\mu^{(2)}=2 n-3\right\}$ (ср., например, с п. 9.2, [37; 6.7$]$ и [38; 2.1$\left.]\right)$ и их плоских моделей $\left\{\mathscr{C}_{\vec{a}}, \vec{a} \in \mathbb{N}^{4}\right\}$. Два других содержат анонсированное полное описание (ср. с $[41 ; \S 3.2]$ ). Все они следуют, более или менее непосредственно, из пш. 9.2-9.5.

9.6. СлЕДСТВИЕ. Пусть $\vec{a} \in \mathbb{N}^{4} u \mu \in \mathbb{M}$ таковьь, что $\vec{a}$ принадлежит множеству $\left\{A^{k}(\mu), B^{k}(\mu), k=0, \ldots, 3\right\}$. Обозначим через $n$ (соответственно $j_{0}$ ) числа $n:=2+\frac{1}{2} \sum_{i=0}^{3} a_{i}\left(a_{i}+1\right)=\frac{1}{2}\left(\mu^{(2)}+3\right)\left(\right.$ соответственно $\left.j_{0}:=\#\left\{i, \mu_{i}=0\right\}\right)$. Тогда

1) $\mathscr{R}(n, \mu)$ является гладким и неприводимьмм (однако пустылм, если $j_{0}=3$, m.e. если $\left.\mu \in \mathbb{M}^{\# 3}\right)$; 
2) естественная проекиия $\mathscr{R}(n, \mu) \rightarrow \mathfrak{X}$ имеет степень $6-2 j_{0}$ и факторизуется при вложении $\mathscr{R}(n, \mu)$ в $\mathscr{C}_{\vec{a}} \backslash\left\{O_{1}, O_{2}, O_{3}\right\}$;

3) проекция $\mathscr{C}_{\vec{a}} \rightarrow\left\{e_{0}=0\right\} \simeq \mathbb{P}^{1}(\mathbb{C})$ (см. доказательство предложсения в $n .7 .7)$ обеспечивает естественную компактификацию $\mathscr{R}(n, \mu) \rightarrow \mathfrak{X} ;$

4) дополнение образа $\mathscr{R}(n, \mu)$ в $\mathscr{C}_{\vec{a}} \backslash\left\{O_{1}, O_{2}, O_{3}\right\}$ также параметризует четные әллиптические конечно-зонные потенциаль, однако ассочиированные с "неканоническими" $\theta$-характеристиками над исключительными накрытиями.

ДокАЗАТЕЛЬство. Очевидно, что достаточно привести доказательство п. 9.6.4). При $\mu \in \mathbb{M}^{\# 0}$ или $\mu \in \mathbb{M}^{\# 3}$ утверждение тривиально (см. пш. 9.2 и 9.5). Рассмотрим произвольное $\mu \in \mathbb{M}^{\# 1}$, для которого известно, что (в общем случае над $\mathfrak{X}$ ) $\# R(n, \mu, X)=4$. Выберем, например, $\mu=\left(0, \mu_{1}, \mu_{2}, \mu_{3}\right)$ (следовательно, $\mu_{0}=0$ и $\left.n=\frac{1}{2}\left(3+\sum_{j=1}^{3} \mu_{j}^{2}\right)\right)$ и обозначим через $\widehat{\pi}:(\widehat{\Gamma}, \widehat{p}) \rightarrow(X, q)$ исключительное накрытие типа $\widehat{\mu}:=\left(2, \mu_{1}, \mu_{2}, \mu_{3}\right)$, арифметического рода $\widehat{g}:=\frac{1}{2}\left(\widehat{\mu}^{(1)}-1\right)=\frac{1}{2}\left(\mu^{(1)}-1\right)+1$ и степени $\frac{1}{2}\left(\widehat{\mu}^{(2)}-1\right)=\frac{1}{2}\left(\mu^{(2)}+3\right)=n$. Пересечение в $S^{\perp}$ между $r_{0}^{\perp}$ и вложением $\widehat{\Gamma}$ в $S^{\perp}$ (п. 2.2) имеет степень, равную $\widehat{\mu}_{0}=2$. Следовательно, $r_{0}^{\perp}$ "пересекает" $\widehat{\Gamma}$ по двум точкам Вейерштрасса, обозначенным через $\widehat{p}_{1}$ и $\widehat{p}_{2}$. Кроме того, непосредственное применение формуль $Ф$ эя-Сигала-Вильсона, такое же как и в пш. 5.3 и 6.3 , показьвает, что эллиптические конечно-зонные потенщиалы, ассоциированные с $\mathscr{O}_{\widehat{\Gamma}}\left((\widehat{g}-2) \widehat{p}+\widehat{p}_{\ell}\right)$ и с $\mathscr{O}_{\widehat{\Gamma}}\left((n+\widehat{g}-2) \widehat{p}+\widehat{p}_{\ell}-\widehat{\pi}^{*}(q)\right)(\ell=1,2)$, и их сдвиги $\left\{\omega_{i}, i=0, \ldots, 3\right\}$ имеют такой же тип (п. 9.1). Таким образом, мы получаем два пропушенных эллиптических конечно-зонных потенциала. Аналогичная конструкция может быть осуществлена и для произвольного $\mu \in \mathbb{M}^{2}$ (см. п. 9.9 и приведенное там доказательство).

9.7. СлЕДСТВИЕ. Для любого $\vec{a} \in \mathbb{N}^{4}$, произвольной әллиптической кривой $X \in \mathfrak{X}$ и произвольной точки $\rho \in X \backslash\left\{\omega_{i}, i=0, \ldots, 3\right\}$ четная функция (6) является әллиптическим конечно-зонным потенциалом тогда и только тогда, когда выполняется (7).

9.8. ЗАмЕчАниЕ. Для того чтобы получить полную картину, следует дополнить п. 9.7 "выгислением" спектральных данных, характеризуюших каждый из описьваемых формулой (6) эллиптический конечно-зонньй потенциал (по крайней мере род соответствуюшей спектральной кривой).

Напомним, что для каждого вектора $\vec{a} \in \mathbb{N}^{4}$ существует единственньй тип $\mu=\mu(\vec{a}) \in \mathbb{M}$ такой, что $\vec{a} \in\left\{A^{k}(\mu), B^{k}(\mu), k=0, \ldots, 3\right\}$ (см., например, [41; $\S 4.4, \S 4.5, \S 5.3])$. Мы также показали (см., например, пш. 7.7, 9.2.iii), 9.3.iii), 9.4.iii)), что, выбирая подходящую каноническую $\theta$-характеристику (например $\xi_{A, 0}$, если $\left.\vec{a}=A^{\circ}(\mu)\right)$ для произвольного элемента из пространства модулей $\mathscr{R}(n, \mu)$ $\left(n:=\frac{1}{2}\left(\mu^{(2)}+3\right)=2+\frac{1}{2} \sum_{i=0}^{3} a_{i}\left(a_{i}+1\right)\right)$, мы получаем собственное вложение

$$
\mathscr{R}(n, \mu) \hookrightarrow \mathscr{C}_{\vec{a}} \backslash\left\{O_{1}, O_{2}, O_{3}\right\}
$$

Как следует из пп. 9.2-9.5, упомянутое "вычисление" позволяет охарактеризовать естественный образ $\mathscr{R}(n, \mu)$ в $\mathscr{C}_{\vec{a}}$. Последний является неприводимой компонентой, равной целой секстике (если $\left.\mu \in \mathbb{M}^{\# 0}\right)$, квартике $\mathscr{Q}_{\vec{a}}\left(\right.$ если $\left.\mu \in \mathbb{M}^{\# 1}\right)$, конике $\mathscr{C}$ оn $\vec{a}$ 
(если $\mu \in \mathbb{M}^{\# 2}$ ) или пустому множеству (если $\mu \in \mathbb{M}^{\# 3}$ ). Соответствуюшие однородные многочлены (в случае $\mu \in \mathbb{M}^{\# 1} \cup \mathbb{M}^{\# 2}$ ) могут быть явно найдены с помошью MAPLE-user Fatma Jeeawock. Мы считаем, однако, более целесообразньм представить более простое описание, основанное на вычислении вектора $\mu(\vec{a})$, а также определяюших многочленов $\mathscr{Q}_{\vec{a}}$ или $\mathscr{C}$ о $n_{\vec{a}}$.

9.9. СлЕДСТВИЕ. Для любого $\vec{a} \in \mathbb{N}^{4}$ обозначим через $\Pi_{\vec{a}}\left(e_{0}, e_{1}, e_{2}\right)$ произведенue

$$
\Pi_{\vec{a}}=R_{\vec{a}}^{+++} \cdot R_{\vec{a}}^{-++} \cdot R_{\vec{a}}^{+-+} \cdot R_{\vec{a}}^{++-}
$$

(см. n. 8.5), через $\mu(\vec{a}) \in \mathbb{M}-$ единственный тип такой, что

$$
\vec{a} \in\left\{A^{k}(\mu(\vec{a})), B^{k}(\mu(\vec{a})), k=0, \ldots, 3\right\},
$$

u через $n-$ число

$$
n:=2+\frac{1}{2} \sum_{i=0}^{3} a_{i}\left(a_{i}+1\right)=\frac{1}{2}\left(\mu(\vec{a})^{(2)}+3\right) .
$$

Тогда для любой эллиптической кривой $X \in \mathfrak{X}$ решения уравнения (7) $(2 \rho \neq 0)$ порождают два различных типа эллиптических конечно-зонных потенииалов $(n .9 .7)$ :

I) $E c л u$

$$
\sum_{i=0}^{3}\left(2 a_{i}+1\right)^{2} \wp^{\prime}\left(\rho-\omega_{i}\right)=0
$$

но $\Pi_{\vec{a}}\left(\wp(\rho), e_{1}, e_{2}\right) \neq 0$, то эллиптический конечно-зонный потенииал $u(x)(6)$ ассочиированныц $c\left(\Gamma, p, \lambda_{\pi}, \xi\right)$, әде $\pi \in R(n, \mu(\vec{a}), X)$ u $\xi$ - одна из канонических $\theta$-характеристик $\left(\xi=\xi_{A, 0}\right.$, если $\vec{a}=A^{\circ}(\mu(\vec{a})), u$ m. д. $)$. $B$ частности, арифметический род спектральной кривой Г равен

$$
g:=\frac{1}{2}\left(\mu(\vec{a})^{(1)}-1\right)=\frac{1}{2} \max \left\{2 M, 1+\Sigma-\left(1+(-1)^{\Sigma}\right)\left(m+\frac{1}{2}\right)\right\}
$$

$2 \partial e$

$$
M=\max \left\{a_{i}\right\}, \quad \Sigma=\sum_{i=0}^{3} a_{i} \quad u \quad m=\min \left\{a_{i}\right\}
$$

II) $E c л u$

$$
\sum_{i=0}^{3}\left(2 a_{i}+1\right)^{2} \wp^{\prime}\left(\rho-\omega_{i}\right)=0 \quad u \quad \Pi_{\vec{a}}\left(\wp(\rho), e_{1}, e_{2}\right)=0
$$

то существуют $k \in\{0, \ldots, 3\}$, исключительное накрытие $\widehat{\pi}:(\widehat{\Gamma}, \widehat{p}) \rightarrow$ $(X, q)$ степени $n$ и типа $\widehat{\mu}\left(\right.$ де $\left.\widehat{\mu}_{i}=\mu(\vec{a})_{i}+2 \delta_{i k}, i=0, \ldots, 3\right)$ и точка Вейерштрасса $\widehat{p}^{\prime} \in \widehat{\Gamma}$ над $\omega_{k}$ такие, что эллиптический конечно-зонный потенциал и $(x)(6)$ ассочиирован с $\left(\widehat{\Gamma}, \widehat{p}, \lambda_{\widehat{\pi}}, \xi\right)$, где $\xi \otimes \mathscr{O}_{\widehat{\Gamma}}\left(\widehat{p}-\widehat{p}^{\prime}\right)-$ одна из канонических $\theta$-характеристик (например, $\xi=\xi_{A, 0} \otimes \mathscr{O}_{\widehat{\Gamma}}\left(\widehat{p}^{\prime}-\widehat{p}\right)$, если 
$\left.\vec{a}=A^{\circ}(\mu(\vec{a}))\right)$. В частности, арифметический род спектральной кривой $\widehat{\Gamma}$ равен

$$
\widehat{g}:=\frac{1}{2}\left(\widehat{\mu}^{(1)}-1\right)=g+1=1+\frac{1}{2} \max \left\{2 M, 1+\Sigma-\left(1+(-1)^{\Sigma}\right)\left(m+\frac{1}{2}\right)\right\} .
$$

Другими словами, функиия (6) является порождающим потенциалом ( $n .5 .7)$ тогда и только тогда, когда выполняется (7),

$$
\Pi_{\vec{a}}\left(\wp(\rho), e_{1}, e_{2}\right) \neq 0, \quad a_{0}=M \quad u \quad 2 M \geqslant \Sigma-\left(1+(-1)^{\Sigma}\right) m .
$$

ДоКАЗАТЕЛЬСТВО. Все, что еще остается доказать - это то, что обращение в нуль $\Pi_{\vec{a}}$ различает образ $\mathscr{R}(n, \mu(\vec{a}))$ и его дополнение в $\mathscr{C}_{\vec{a}} \backslash\left\{O_{1}, O_{2}, O_{3}\right\}$. Ранее показано, что пересечение $\left(\mathscr{C}_{\vec{a}} \backslash\left\{O_{1}, O_{2}, O_{3}\right\}\right) \cap\left\{\Pi_{\vec{a}}=0\right\}$ содержит упомянутое дополнение (см. пш. 9.3.iii), 9.4.iii) и 9.5.iii)). С другой стороны, можно легко проверить, что для любых $R \in\left\{R_{\vec{a}}^{+++}, R_{\vec{a}}^{-++}, R_{\vec{a}}^{+-+}, R_{\vec{a}}^{++-}\right\}$коника $\{R=0\}$ пересекает секстику $\mathscr{C}_{\vec{a}}$ в $O_{j}(j=$ $1,2,3)$ с кратностью 4. Следовательно, $\{R=0\}$ не пересекает ни одной неприводимой компоненты $\mathscr{C}_{\vec{a}}$ (отличной от $\{R=0\}$ ) вне $\left\{O_{1}, O_{2}, O_{3}\right\}$. Таким образом, мы показали, что $\{R=0\}$, а следовательно, и $\left\{\Pi_{\vec{a}}=0\right\}$ не пересекается с образом $\mathscr{R}(n, \mu(\vec{a}))$, как и требовалось.

Сделаем в заключение несколько замечаний относительно типа $\widehat{\mu}$ и объясним выбор “неканонических" $\theta$-характеристик в случае п. 9.9.II). Напомним, что дополнение к образу $\mathscr{R}(n, \mu(\vec{a}))$ непусто тогда и только тог да, ког да $\mu(\vec{a})$ принадлежит $\bigcup_{j=1}^{3} \mathbb{M} \# j$. Случаи $\mu(\vec{a}) \in \mathbb{M}^{\# 1}$ и $\mu(\vec{a}) \in \mathbb{M}^{\# 3}$ были уже изучены полностью (см. пп. 9.6.4) и 6.6). Предположим, наконец, что $\mu(\vec{a}) \in \mathbb{M}^{\# 2}$. Предположим, для простоты, что $\mu(\vec{a}):=$ $(2 \ell+1,2 k, 0,0)(k \neq 0)$ и $A^{\circ}(\mu(\vec{a}))=\vec{a}$. Пусть $\widehat{\pi}:(\widehat{\Gamma}, \widehat{p}) \rightarrow(X, q)$ обозначает исключительное накрытие типа $\widehat{\mu}:=(2 \ell+1,2 k, 2,0)($ соответственно $\widehat{\mu}:=(2 \ell+1,2 k, 0,2))$, степени $n:=\frac{1}{2}\left(\widehat{\mu}^{(2)}+1\right)=\frac{1}{2}\left(\mu(\vec{a})^{(2)}+3\right)$ и арифметического рода $\widehat{g}:=\frac{1}{2}\left(\mu(\vec{a})^{(1)}+1\right)$, и пусть $\widehat{p}^{\prime}$ обозначает одну из двух точек Вейерштрасса кривой $\widehat{\Gamma}$ над $\omega_{2}$ (соответственно над $\left.\omega_{3}\right)$. Непосредственное применение формулы для нижних границ (п. 5.4), как и в доказательстве теоремы в п. 6.3, приводит к заключению, что эллиптический конечно-зонньй потенциал, ассоциированньй с

$$
\xi_{A, 0} \otimes \mathscr{O}_{\widehat{\Gamma}}\left(\widehat{p}^{\prime}-\widehat{p}\right)=\mathscr{O}_{\widehat{\Gamma}}\left((\widehat{g}-2) \widehat{p}+\widehat{p}^{\prime}\right),
$$

равен $u(x)(6)$ для некоторого $\rho \in X(2 \rho \neq 0)$, удовлетворяюшего п. 9.9.II). Следовательно, каждый из вариантов, $\widehat{\mu}=(2 \ell+1,2 k, 2,0)$ и $\widehat{\mu}=(2 \ell+1,2 k, 0,2)$, приводит к паре эллиптических конечно-зонных потенциалов, соответствуюших слою над $X \in \mathfrak{X}$ каждой коники, содержащейся в $\mathscr{C}_{\vec{a}} \backslash \mathscr{A}^{\circ}(\mathscr{R}(n, \mu(\vec{a})))$, дополнении к образу $\mathscr{R}(n, \mu(\vec{a}))$. 


\section{СПИСОК ЛИТЕРАТУРЫ}

[1] H. Airault, H. P. Mc Kean, J. Moser. Rational and elliptic solutions of the Korteweg-de Vries equation and a related many body problem // Comm. Pure Appl. Math. 1977. V. 30. P. $95-148$.

[2] A. Altman, S. Kleiman. Compactifying the Picard scheme // Adv. in Math. 1980. V. 35. P. $50-112$.

[3] М. В. Бабич, А. И. Бобенко, В.Б.Матвеев. Редукция тэта-функций Римана рода $g$ к тэта-функциям младших родов и симметрии алгебраических кривых // Докл. АН СССР 1983. T. 272. № 1. C. 13-17.

[4] М.В. Бабич, А.И. Бобенко, В.Б. Матвеев. Решения нелинейных уравнений, интегрируемых методом обратной задачи, в тэта-функциях Якоби и симметрии алгебраических кривых // Изв. АН СССР. Сер. матем. 1985. Т. 49. С. 511-529.

[5] Е.Д. Белоколос, В.З. Энольский. Эллиптические солитоны Вердье и теория редукции Вейерштрасса // Функц. анализ и его прил. 1989. V. 23. №1. Р. 57-58.

[6] E.D. Belokolos, V.Z. Enolskii. Reduction of theta functions and elliptic finite-gap potentials // Acta Appl. Math. 1994. V. 36. P. 87-117.

[7] Е.Д. Белоколос, А. И. Бобенко, В.З. Энольский, В.Б. Матвеев. Алгеброгеометрические принципы суперпозиции конечно-зонных решений интегрируемых нелинейных уравнений // УМН. 1986. Т. 41. № 2. С. 3-42.

[8] R. Dickson, F. Gesztesy, K. Unterkofler. A new approach to the Boussinesq hierarchy // Math. Nachr. 1999. V. 198. P. 51-108.

[9] R. Dickson, F. Gesztesy, K. Unterkofler. Algebro-geometric solutions of the Boussinesq hierarchy // Rev. Math. Phys. 1999. V. 11. № 7. P. 823-879.

[10] Б. А. Дубровин. Периодическая задача для уравнения Кортевега-де Вриза в классе конечно-зонных потенциалов // Функц. анализ и его прил. 1975. Т. 9. № 3. С. 41-51.

[11] Б. А. Дубровин. Тэта-функции и нелинейные уравнения. (С приложением И. М. Кричевера.) // УМН. 1981. Т. 36. № 2. С. 11-80.

[12] Б. А. Дубровин, В.Б. Матвеев, С. П. Новиков. Нелинейные уравнения типа Кортевега-де Вриза, конечно-зонные операторы и абелевы многообразия // УМН. 1976. Т. 31. № 1. C. $55-135$.

[13] Б. А. Дубровин, С.П. Новиков. Периодические и условно-периодические аналоги много-солитонных решений уравнения Кортевега-де Вриза // ЖЭТФ. 1974. Т. 67. №6. C. 2131-2144.

[14] V.Z. Enol'skii, N. A. Kostov. On the geometry of elliptic solitons // Acta Appl. Math. 1994. V. 36. № 1-2. P. 57-86.

[15] J. D. Fay. On the even-order vanishing of Jacobian theta functions // Duke Math. J. 1984. V. 51. P. 109-132.

[16] H. Flaschka. On the inverse problem for Hill's operator // Arch. Rational Mech. Anal. 1975. V. 59. P. 293-309.

[17] L. Gavrilov, A. M. Perelomov. On the explicit solutions of the elliptic Calogero system // J. Math. Phys. 1999. V. 40. № 12. P. 6339-6352.

[18] F. Gesztesy, R. Weikard. Treibich-Verdier potentials and the stationary $(m)$ KdV hierarchy // Math. Z. 1995. V. 219. P. 451-476.

[19] F. Gesztesy, R. Weikard. A characterization of elliptic finite-gap potentials // C. R. Acad. Sci. Paris Sér. I Math. 1995. V. 321. P. 837-841.

[20] F. Gesztesy, R. Weikard. Elliptic algebro-geometric solutions of the KdV and AKNS hierarchies - an analytic approach // Bull. Amer. Math. Soc. 1998. V. 35. № 4. P. 271-317.

[21] F. Gesztesy, R. Weikard. Picard potentials and Hill's equation on a torus // Acta Math. 1996. V. 176. P. 73-107.

[22] F. Gesztesy, R. Weikard. On Picard potentials // Differential Integral Equations. 1995. V. 8. №6. P. 1453-1476.

[23] Ф. Грифрфитс, Дж. Харрис. Принципы алгебраической геометрии. М.: Мир, 1982.

[24] G. Halphen. Mémoire sur la réduction des équations différentielles aux formes intégrales // Mem. Pres. Acad. Sci. France. 1884. V. 28. P. 1-300.

[25] C. Hermite. Cuvres. V. 3. Paris: Gauthier-Villars, 1912. 
[26] E. L. Ince. Further investigations into the periodic Lamé equation // Proc. Roy. Soc. Edinburgh. 1940. V. 60. P. 83-99.

[27] А. Р. Итс, В. Б. Матвеев. Оператор Шредингера с конечно-зонным спектром и $N$-солитонные решения уравнения Кортевега-де Вриза // ТМФ. 1975. Т. 23. С. 51-68.

[28] И. М. Кричевер. Алгебро-геометрическая конструкция уравнений Захарова-Шабата и их периодических решений // Докл. АН СССР. 1976. Т. 227. № 2. С. 291-294.

[29] И. М. Кричевер. Методы алгебраической геометрии в теории нелинейных уравнений // УМН. 1977. Т. 32. №6. С. 180-208.

[30] И. М. Кричевер. Эллиптические решения уравнения Кадомцева-Петвиашвили и интегрируемые системы частиц // Функц. анализ и его прил. 1980. Т. 14. С. 45-54.

[31] H.P. McKean, P. van Moerbeke. The spectrum of Hill's equation // Invent. Math. 1975. V. 30. P. 217-274.

[32] С. П. Новиков. Периодическая задача для уравнения Кортевега-де Вриза // Функц. анализ и его прил. 1974. Т. 8. С. 236-246.

[33] G. Segal, G. Wilson. Loop groups and equations of KdV type // Inst. Hautes Études Sci. Publ. Math. 1985. V. 61. P. 5-65.

[34] А.О. Смирнов. Эллиптические решения уравнения Кортевега-де Вриза // Матем. заметки. 1989. Т. 45. С. $66-73$.

[35] A. O. Smirnov. Finite-gap elliptic solutions of the KdV equation // Acta Appl. Math. 1994. V. 36. № 1-2. P. 125-166.

[36] A. Treibich. Tangential polynomials and elliptic solitons // Duke Math. J. 1989. V. 59. №3. P. 611-627.

[37] A. Treibich. New elliptic potentials // Acta Appl. Math. 1994. V. 36. P. 27-48.

[38] A. Treibich, J. L. Verdier. Solitons elliptiques. (With an appendix by J. Oesterle.) // Progr. in Math. 1990. V. 88. P. 437-480.

[39] A. Treibich, J.L. Verdier. Variétés de Kritchever des solitons elliptiques de KP // Proceedings of the Indo-French Conference on Geometry, Bombay 1989 / ed. S. Ramanan et al. Delhi: Hindustan Book Agency, 1993. P. 187-232.

[40] A. Treibich, J. L. Verdier. Revêtements tangentiels et sommes de 4 nombres triangulaires // C. R. Acad. Sci. Paris Sér. I Math. 1990. V. 311. P. 51-54.

[41] A. Treibich, J. L. Verdier. Revêtements exceptionnels et sommes de 4 nombres triangulaires // Duke Math. J. 1992. V. 68. № 2. P. 217-236.

[42] K. Unterkofler. On the solution of Halphen's equation // Differential Integral Equations. 2001. V. 14. № 9. P. 1025-1050.

\section{Предметный указатель}

вложение Абеля п. 1.3; функция Бейкера-Ахиезера п. 1.1; исключительное накрытие п. 4.3; исключительньй дивизор п. $4.1 ; n$-подходящий вектор п. 2.4 ; гиперэллиптическая инволюция п. 6.7 ; гиперэллиптическая отмеченная кривая п. 1.2 ; эллиптический конечно-зонньй потенщиал п. 1.13; компактифицированный якобиан п. 1.6; обобщенньй якобиан п. 1.3; конечно-зонньй потенщиал п. 1.2 ; (n-й) КдВ-локус п. 1.8.2); пространство модулей эллиптических кривых $(\mathfrak{X})$ п. 7.6; пространство модулей компактифицированных якобианов $(\mathscr{W}(n, \mu)$ и $\mathscr{W}(n))$ п. 7.7 ; непримитивная функция п. 1.13 ; орбитньй морфизм п. 1.7.3); частичное упорядочение $((m, \nu) \prec(n, \mu))$ п. 4.5; плоская модель п. 7.4; порождающий потенщиал п. 5.7; касательное накрытие п. 1.4 ; гиперэллиптическое касательное накрытие п. 1.4; минимально-гиперэллиптическое касательное накрытие п. 1.9; критерий касательности п. 1.5; касательная функция п. 1.5; $\theta$-характеристика п. 1.6; каноническая $\theta$-характеристика $\left\{\xi_{A, k}, \xi_{B, k}(k=0, \ldots, 3)\right\}$ п. 5.3.3); тэта-дивизор п. 1.7.3); тип $\pi$ п. 2.1; универсальная эллиптическая кривая п. 7.6; ю-функция Вейерштрасса п. 1.8.2). 


\section{Указатель обозначений}

п. $1.1 \psi_{D}(x, y, t ; \lambda), \xi_{j, D}(x, y, t)$;

п. $1.2 u_{D}(x, y, t)$;

п. $1.3 A_{p}:(\Gamma, p) \rightarrow \mathrm{Jac} \Gamma, i_{\pi}, \Lambda$;

п. $1.6 W(\Gamma), \omega_{\Gamma}, \mathscr{L}^{*}$;

п. $1.7 \Theta_{\Gamma}, \operatorname{Orb}_{\mathscr{L}}: X \rightarrow W(\Gamma), X_{n}, \Gamma^{\circ}, \Gamma_{g}^{\circ}, I_{\pi}: W(\Gamma) \rightarrow X_{n} ;$

п. $1.8 \wp, \lambda_{\pi}, \operatorname{KdV}(n, X)$;

п. $1.12 W(n, X), R(n, X), I_{n}(X): W(n, X) \rightarrow \operatorname{KdV}(n, X)$;

п. $2.1 \mathscr{E}, S, C_{0}, \pi_{S}: S \rightarrow X, \tau, s_{i}, r_{i}(i=0, \ldots, 3), S^{\perp}, \pi_{S^{\perp}}: S^{\perp} \rightarrow X$, $\tau^{\perp}, s_{i}^{\perp}, r_{i}^{\perp}(i=0, \ldots, 3), e: S^{\perp} \rightarrow S, S^{\sim}, \phi: S^{\perp} \rightarrow S^{\sim}$;

п. $2.2 i^{\perp}, \mu, \mu^{(1)}, \mu^{(2)}$;

п. $2.3 S_{0}$;

п. $2.5 \lambda(n, \mu)$

п. $2.8 R(n, \mu, X)$;

п. $3 \ell, \ell^{\sim}, s_{i}^{\sim}, r_{i}^{\sim}(i=0, \ldots, 3)$;

п. $3.1 S_{i}^{\sim}(i=0, \ldots, 3)$;

п. $3.3 K, K^{\perp}, K^{\sim}$;

п. $4.1 C_{\mu}^{\sim}$;

п. $4.3 C_{\mu}^{\perp}, p_{\mu}$;

п. $4.11 \chi_{y}$;

п. $5.3 \xi_{A, i}, \xi_{B, i}(i=0, \ldots, 3)$;

п. $5.4 m_{i}^{A, 0}, m_{i}^{B, 0}(i=0, \ldots, 3)$;

п. $6.4 \mathbb{M}, \mathbb{M}^{\# i}, A^{i}, B^{i}: \mathbb{M} \rightarrow \mathbb{N}^{4}(i=0, \ldots, 3)$;

п. $7.4 \mathbb{H}, \mathscr{C}_{\vec{a}}\left(\vec{a} \in \mathbb{N}^{4}\right)$;

п. $7.6 \mathfrak{X}, \mathscr{R}(n, \mu), \mathscr{A}^{i}, \mathscr{B}^{i}: \mathscr{R}(n, \mu) \rightarrow \mathscr{C}_{\vec{a}}(i=0, \ldots, 3), O_{1}, O_{2}, O_{3}$;

п. $7.7 \mathscr{W}(n, \mu), \mathscr{K} d \mathscr{V}(n), \overline{\mathfrak{X}} \rightarrow \mathfrak{X},(\overline{\mathfrak{X}} / \mathfrak{X})_{n}, \mathscr{W}(n), \mathscr{I}_{n}$;

п. $8.1 P(\alpha, \beta, \gamma), F_{\vec{a}}\left(e_{0}, e_{1}, e_{2}, e_{3}\right)\left(\vec{a} \in \mathbb{N}^{4}\right), \varphi$ : $\mathbb{H} \rightarrow \mathbb{H}$;

п. $8.2 E_{j}(j=1,2,3)$;

п. $8.3 \widehat{\widehat{\mathbb{H}}}$;

п. $8.5 \widehat{\widehat{C}}_{\vec{a}}, R_{\vec{a}}^{+++}, R_{\vec{a}}^{-++}, R_{\vec{a}}^{+-+}, R_{\vec{a}}^{++-}\left(\vec{a} \in \mathbb{N}^{4}\right)$;

п. $8.6 \mathscr{C}_{\text {on }}, \mathscr{C}_{\vec{a}}^{++}+\mathscr{C}_{\vec{a}}^{-++}, \mathscr{C}_{\vec{a}}^{+-+}, \mathscr{C}_{\vec{a}}^{++-}, \widehat{\hat{\mathscr{C}}}_{\vec{a}}^{+++}, \widehat{\hat{\mathscr{C}}}_{\vec{a}}^{-++}, \widehat{\hat{\mathscr{C}}}_{\vec{a}}^{+-+}, \widehat{\hat{\mathscr{C}}}_{\vec{a}}^{++-}$ $\left(\vec{a} \in \mathbb{N}^{4}\right)$

п. $9.3 \mathscr{Q}_{\vec{a}}$;

п. $9.9 \prod_{\vec{a}}$. 